\title{
Corrections to Yukawa couplings from higher dimensional operators in a natural SUSY $\mathrm{SO}(10)$ and HL-LHC implications
}

\author{
Amin Aboubrahim, ${ }^{a, b}$ Pran Nath $^{b}$ and Raza M. Syed ${ }^{b, c, 1}$ \\ ${ }^{a}$ Institut für Theoretische Physik, Westfälische Wilhelms-Universität Münster, \\ Wilhelm-Klemm-Straße 9, 48149 Münster, Germany \\ ${ }^{b}$ Department of Physics, Northeastern University, Boston, MA 02115-5000, U.S.A. \\ ${ }^{c}$ Department of Physics, American University of Sharjah, P.O. Box 26666, Sharjah, U.A.E. \\ E-mail: aabouibr@uni-muenster.de, p.nath@northeastern.edu, \\ rsyedQaus.edu
}

ABSTRACT: We consider a class of unified models based on the gauge group $\mathrm{SO}(10)$ which with appropriate choice of Higgs representations generate in a natural way a pair of light Higgs doublets needed to accomplish electroweak symmetry breaking. In this class of models higher dimensional operators of the form matter-matter-Higgs-Higgs in the superpotential after spontaneous breaking of the GUT symmetry generate contributions to Yukawa couplings which are comparable to the ones from cubic interactions. Specifically we consider an $\mathrm{SO}(10)$ model with a sector consisting of $126+\overline{126}+210$ of heavy Higgs which breaks the GUT symmetry down to the standard model gauge group and a sector consisting of $2 \times 10+120$ of light Higgs fields. In this model we compute the corrections from the quartic interactions to the Yukawa couplings for the top and the bottom quarks and for the tau lepton. It is then shown that inclusion of these corrections to the GUT scale Yukawas allows for consistency of the top, bottom and tau masses with experiment for low $\tan \beta$ with a value as low as $\tan \beta$ of $5-10$. We compute the sparticle spectrum for a set of benchmarks and find that satisfaction of the relic density is achieved via a compressed spectrum and coannihilation and three sets of coannihilations appear: chargino-neutralino, stop-neutralino and stau-neutralino. We investigate the chargino-neutralino coannihilation in detail for the possibility of observation of the light chargino at the high luminosity LHC (HL-LHC) and at the high energy LHC (HE-LHC) which is a possible future $27 \mathrm{TeV}$ hadron collider. It is shown that all benchmark models but one can be discovered at HL-LHC and all would be discoverable at HE-LHC. The ones discoverable at both machines require a much shorter time scale and a lower integrated luminosity at HE-LHC.

Keywords: Phenomenology of Field Theories in Higher Dimensions

ARXiv EPRINT: 2005.00867

${ }^{1}$ Permanent address is $\mathrm{c}$. 


\section{Contents}

1 Introduction 1

2 The model 3

3 Yukawa couplings from cubic interactions 5

4 Corrections to Yukawa couplings from higher dimensional operators $\quad 6$

5 Analysis of model implications $\quad 8$

$\begin{array}{lll}5.1 & \text { Electroweakino pair production at the LHC and their decay channels } & 13\end{array}$

$\begin{array}{lll}5.2 & \text { Signal and background simulation and event selection } & 15\end{array}$

$\begin{array}{lll}\text { 5.3 Cut implementation and the estimated integrated luminosity } & 17\end{array}$

$\begin{array}{llr}6 & \text { Conclusion } & 19\end{array}$

A Notation and decomposition of $\mathrm{SU}(5)$ fields in terms of $\mathrm{SU}(3)_{C} \times \mathrm{SU}(2)_{L} \times$ $\begin{array}{ll}\mathrm{U}(1)_{Y} & 21\end{array}$

B Breaking the SO(10) gauge symmetry 22

$\begin{array}{ll}\text { C Higgs doublet mass matrix } & 24\end{array}$

D Details of Yukawa couplings from quartic interactions 25

\section{Introduction}

Grand unified models based on $\mathrm{SO}(10)[1,2]$ are the most desirable of grand unified models as they provide unification of the standard model gauge group and a unification of one generation of matter consisting of quarks and leptons in a single irreducible representation. The Higgs sector of $\mathrm{SO}(10)$ models is very rich consisting of several possible representations which can be used to break the grand unified symmetry down to the standard model gauge group. Some of these consist of $16+\overline{16}, 45,54,126+\overline{126}, 210$ among others. In this work we will focus on large Higgs representations to break the grand unified theory (GUT) symmetry for reasons explained below. Large representations have been used in the literature for quite some time, a small sample of which are [3-5] and for some more recent works see, e.g., [6-12] and the references therein. However, in grand unified models with small as well as with large Higgs represenations the Higgs doublets lie in irreducible representations of the unified gauge group along with other components which carry color, such as color triplets. The super-partners of these enter in proton decay (for a review see [13]) and they must be very heavy, i.e., of the GUT scale size, which makes the Higgs doublets also 
superheavy and thus unsuitable for electroweak symmetry breaking. One can, of course, manufacture a light Higgs doublet pair by fine tuning which, however, is rather large.

It is more appealing to have models where some higher symmetry, a group theoretic constraint, or a vacuum selection constraint leads to a pair of light Higgs doublets. Such unified models may be viewed as natural, and GUT models which exhibit this property may be viewed as natural GUTs. In string theory examples of such models exist, see e.g., [1416] and for natural GUTs see [17]. Natural GUT models may also be realized in the framework of field theory. Thus the Dimopoulos-Wilczek mechanism allows for generation of light Higgs doublets in $\mathrm{SO}(10)$ [18-21]. Another possibility to generate a light vector-like Higgs doublet is by a combination of Higgs representations. In SU(5) one finds $[22,23]$ that a combination of $5+\overline{5}, 50+\overline{50}$ and 75 of Higgs conspire to make the color Higgs triplets all heavy but leaves one pair of Higgs doublets light. A similar phenomenon occurs in $\mathrm{SO}(10)[24,25]$ where a pair of light Higgs doublets can arise purely by a proper combination of heavy and light Higgs multiplets. Models of this type are referred to as missing partner models and they belong to the larger class of natural models as defined above. This last class of models involve Higgs fields in large tensor and spinor representations. ${ }^{1}$

The mechanism that operates in natural field theoretic GUT models is the following: suppose the GUT model consists of two types of Higgs fields, where one set is heavy and the other set is light. Let us further suppose the heavy sector possesses $n_{D}^{H}$ number of Higgs doublet pairs, and the light sector possesses $n_{D}^{L}$ number of Higgs doublet pairs and $n_{D}^{L}>n_{D}^{H}$. In this case if the light and the heavy sectors mix, $n_{D}^{H}$ number of light Higgs doublet pairs will become heavy leaving $n_{D}^{L}-n_{D}^{H}$ number of Higgs doublet pairs light. In the class of models we consider $n_{D}^{L}-n_{D}^{H}=1$ and thus one naturally produces one pair of light Higgs doublets which is desired for electroweak symmetry breaking. At the same time we need to make sure that the number of color triplets/anti-triplets $n_{T}^{H}$ in the heavy sector and the number of color triplets/anti-triplets $n_{T}^{L}$ in the light sector match, i.e., $n_{T}^{L}-n_{T}^{H}=0$ which makes all the color Higgs triplets/anti-triplets heavy when the light and the heavy sectors mix.

It is of interest to investigate physics implications of $\mathrm{SO}(10)$ models of this type. Thus proton stability in these models has been discussed in [26]. Here we will discuss quark-charged lepton masses and the sparticle spectrum in a class of these models and also investigate the implications for supersymmetry (SUSY) discovery at the HL-LHC and HE-LHC. In this work we will consider one specific model where the heavy sector consists of $126+\overline{126}+210$ of Higgs fields and the light sector consists of $2 \times 10+120$ of Higgs fields. In this case using the counting discussed above only one Higgs doublet pair remains light while all the color triplet/anti-triplet pairs become heavy. An important result of our analysis is to show that in models of this type, higher dimensional operators can generate contributions to Yukawa couplings which are comparable to the contributions from the cubic interactions. The reason for this is the following: the quartic interactions of the type matter-matter-light Higgs- heavy Higgs suppressed by a heavy (cutoff) mass produce contributions comparable to those from the cubic interactions after spontaneous breaking of

\footnotetext{
${ }^{1}$ An example of a natural GUT model with spinor Higgs representations is the case when the heavy Higgs consists of $560+\overline{560}$ and the light Higgs consists of $2 \times 10+320$ [25].
} 
the GUT symmetry. This is so because one of the heavy Higgs fields, i.e., the 210-plet, has a large vacuum expectation value (VEV) and thus can make a non-negligible contribution even when suppressed by the cutoff mass.

The outline of the rest of the paper is as follows: in section 2 we give a description of the model. In section 3 we compute the contribution to Yukawas for the third generation from the cubic interactions. In section 4 we give computations of the Yukawa couplings which arise from the cubic matter-matter-Higgs interactions and from the quartic matter-matterHiggs-Higgs interactions where one of the Higgs fields belongs either to the 10-plets or to the 120-plet while the other Higgs field is the 210-plet. After spontaneous symmetry breaking at the GUT scale these quartic interactions contribute to the Yukawa couplings. We show that the quartic superpotential corrections to the Yukawa couplings can be substantial and can modify the well known constraint that for the $t-b-\tau$ unification one needs a large $\tan \beta$ [27]. In section 5 we give a numerical estimate of the VEVs of the heavy fields which break the $\mathrm{SO}(10)$ symmetry down to the standard model gauge group. This is done for a set of benchmarks for the parameters involving the heavy fields. In this section we also give the numerical computations of the contributions of the quartic operators in the Yukawa couplings. Implications of the model at high luminosity LHC (HL-LHC) regarding the possible observation of supersymmetry in this model are also discussed. Conclusions are given in section 6. Several appendices are also included. Thus notation of the model is given in appendix A where we also give a decomposition of the relevant irreducible representations of $\mathrm{SO}(10)$ in irreducible representations of $\mathrm{SU}(5)$. In appendix $\mathrm{B}$ we discuss spontaneous breaking of the $\mathrm{SO}(10)$ symmetry by the heavy Higgs fields $126+\overline{126}$ and 210 . In appendix $\mathrm{C}$ we exhibit for completeness the $7 \times 7$ Higgs doublet mass matrix as a result of the mixing of the heavy fields $126+\overline{126}+210$ with the light fields $2 \times 10+120$ discussed in section 2 . In appendix D details of the computation of the contributions of quartic interactions to Yukawa couplings are given.

\section{The model}

The heavy Higgs sector of our $\mathrm{SO}(10)$ model consists of $126(\Delta)+\overline{126}(\bar{\Delta})+210(\Phi)$, while the light sector contains $\sum_{r=1}^{2} 10_{r}\left({ }^{r} \Omega\right)+120(\Sigma)$. This particular particle content in the Higgs sector gives after mixing of the light and heavy sectors just a pair of light Higgs doublets [25, 28]. Finally, the Yukawa sector contains a single (third) generation of quarks and leptons that reside in the $16\left(\Psi_{(+)}\right)$multiplet spinor representation. The GUT symmetry is broken via the superpotential [28]

$$
\begin{aligned}
W_{\mathrm{GUT}}= & M^{126} \Delta_{\mu \nu \rho \sigma \lambda} \bar{\Delta}_{\mu \nu \rho \sigma \lambda}+M^{210} \Phi_{\mu \nu \rho \sigma} \Phi_{\mu \nu \rho \sigma}+\eta \Phi_{\mu \nu \rho \sigma} \Delta_{\mu \nu \lambda \tau \xi} \bar{\Delta}_{\rho \sigma \lambda \tau \xi} \\
& +\lambda \Phi_{\mu \nu \rho \sigma} \Phi_{\rho \sigma \lambda \tau} \Phi_{\lambda \tau \mu \nu} .
\end{aligned}
$$

Here the VEVs of the $126+\overline{126}$ fields, i.e., $\mathcal{V}_{1_{126}}$ and $\mathcal{V}_{1_{\overline{126}}}$ and the VEVs of the 210-plet fields $\mathcal{V}_{1_{210}}, \mathcal{V}_{24_{210}}, \mathcal{V}_{75_{210}}$ break the GUT symmetry down to the gauge group symmetry of $\mathrm{SO}(10)$. Details of this breaking are given in appendix B. Next we discuss the generation of the light Higgs doublet. The couplings appearing in the superpotential that generate a 
light Higgs doublet pair are [28]

$$
\begin{aligned}
W_{\mathrm{DT}}= & a{ }^{1} \Omega_{\mu} \bar{\Delta}_{\mu \nu \rho \sigma \lambda} \Phi_{\nu \rho \sigma \lambda}+\sum_{r=1}^{2} b_{r}{ }^{r} \Omega_{\mu} \Delta_{\mu \nu \rho \sigma \lambda} \Phi_{\nu \rho \sigma \lambda}+c \Sigma_{\mu \nu \rho} \Delta_{\nu \rho \sigma \lambda \tau} \Phi_{\mu \sigma \lambda \tau} \\
& +\bar{c} \Sigma_{\mu \nu \rho} \bar{\Delta}_{\nu \rho \sigma \lambda \tau} \Phi_{\mu \sigma \lambda \tau} .
\end{aligned}
$$

In writing eqs. (2.1) and (2.2) we have imposed the condition that there be a light sector consisting of $2 \times 10+120$. This condition requires absence of explicit masses for $2 \times 10+120$ as well as absence of couplings $10 \cdot 120 \cdot 210$ and $120 \cdot 120 \cdot 210$ which would otherwise give superheavy masses to them after the 210 develops a heavy VEV. These constraints which were given in eqs. (11) and (12) of [28] are needed to guarantee the existence of a light sector which is necessary for the missing partner mechanism to operate. The $\mathrm{SO}(10)$ heavy Higgs multiplets $126+\overline{126}+210$ contain three heavy $\mathrm{SU}(2)$ doublet pairs:

$$
\left.\left.\left\{{ }^{(5 \overline{126}}\right) \mathrm{D}^{a},{ }^{\left({ }_{126}\right)} \mathrm{D}_{a}\right\}, \quad\left\{{ }^{\left(45_{126}\right)} \mathrm{D}^{a}, \quad{ }^{(\overline{45}} \overline{126}\right) \mathrm{D}_{a}\right\}, \quad\left\{{ }^{\left(5_{210}\right)} \mathrm{D}^{a},{ }^{\left(\overline{5}_{210}\right)} \mathrm{D}_{a}\right\}
$$

The $\mathrm{SO}(10)$ light Higgs multiplets $2 \times 10+120$ contain four light $\mathrm{SU}(2)$ doublet pairs:

$$
\left\{{ }^{\left({ }_{10} 0_{1}\right)} \mathrm{D}^{a},\left({ }^{\left({ }_{10} 0_{1}\right.}\right) \mathrm{D}_{a}\right\}, \quad\left\{{ }^{\left(5_{10} 0_{2}\right)} \mathrm{D}^{a},\left({ }^{\left(5_{10}\right.}\right) \mathrm{D}_{a}\right\}, \quad\left\{{ }^{\left(5_{120}\right)} \mathrm{D}^{a},\left({ }^{\left({ }_{120}\right)} \mathrm{D}_{a}\right\}, \quad\left\{{ }^{\left(45_{120}\right)} \mathrm{D}^{a},\left({ }^{\left({ }_{5} 120\right.}\right) \mathrm{D}_{a}\right\}\right.
$$

Because of the mixings of the heavy Higgs and light Higgs sectors via eq. (2.2), three linear combinations of the four light Higgs doublet pairs in eq. (2.4) mix with the three heavy Higgs doublet pairs of eq. (2.3) and become heavy leaving only one pair of Higgs doublets light. This light Higgs doublet is the one that enters the electroweak symmetry breaking. The specific linear combination of the seven Higgs doublet pairs that yield a light Higgs doublet pair can be gotten by diagonalizing the $7 \times 7$ Higgs doublet mass matrix given in appendix C. The doublet mass matrix is diagonalized by two unitary matrices $U$ and $V$ whose relevant elements are displayed in eq. (2.5),

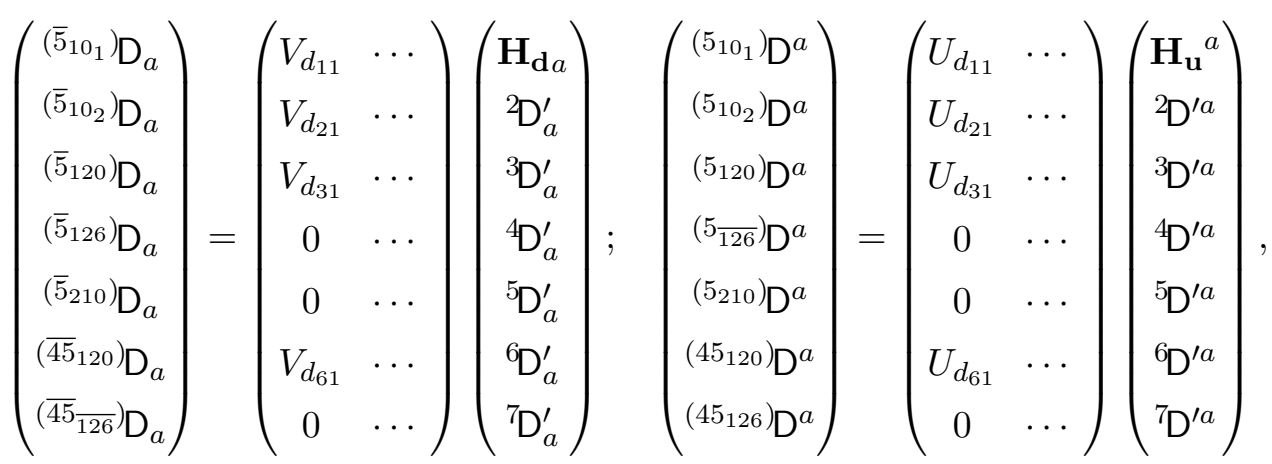

where D's and D's represent the normalized kinetic energy basis and normalized kinetic and mass eigenbasis, respectively of the doublet mass matrix of eq. (C.1). The pair of doublets $\left(\mathbf{H}_{\mathbf{d} a}, \mathbf{H}_{\mathbf{u}}{ }^{a}\right)$ are identified to be light and are the normalized electroweak Higgs doublets of the minimal supersymmetric standard model (MSSM). The matrix elements of $U$ and $V$ marked by dots do not contribute in the low energy theory. Numerical values of the nonzero matrix elements of $U$ and $V$ are displayed in tables 2 and 3 for benchmarks of table 1 . 


\section{Yukawa couplings from cubic interactions}

Since the product $16 \times 16=10_{s}+120_{a}+\overline{126}_{s}$, the 16-plet of matter has couplings with the 10-plet, 120-plet and 126-plet of Higgs. Here the subscripts $a$ and $s$ indicate if the Higgs tensor appears symmetrically or anti-symmetrically under the exchange of two 16's. Since the 126 field is superheavy it does not contribute to the fermion cubic couplings as can be seen from eq. (2.5), where one has $U_{d_{41}}=0$ and $V_{d_{71}}=0$. The 120-plet couplings are anti-symmetric in the generation space and so they also do not contribute because we consider here only one, i.e., the third generation. Thus only the $16-16-10$ cubic couplings contribute to the Yukawa couplings and their computation follows from

$$
W_{3}=\sum_{r=1}^{2} f^{10_{r}}\left\langle\Psi_{(+)}^{*}\left|B \Gamma_{\mu}\right| \Psi_{(+)}\right\rangle^{r} \Omega_{\mu}
$$

Here $B$ and $\Gamma$ 's are the $\mathrm{SO}(10)$ charge conjugation and gamma matrices [6]. The decomposition of an $\mathrm{SO}(10)$ vertex in the $\mathrm{SU}(5)$ basis using the oscillators [30] and the techniques developed in $[6-8,31]$ allow us to compute particle content in the $\mathrm{SU}(3)_{C} \times \mathrm{SU}(2) \times \mathrm{U}(1)_{Y}$ basis. Thus for $W_{3}$ in $\mathrm{SU}(5)$ decomposition we get

$$
\begin{aligned}
& W_{3}=i \sum_{r=1}^{2} f^{10_{r}} {\left[2 \sqrt{2} \mathrm{M}^{i j} \mathrm{M}_{i} \mathrm{H}_{j}^{\left(10_{r}\right)}+\frac{1}{2 \sqrt{2}} \epsilon_{i j k l m} \mathrm{M}^{i j} \mathrm{M}^{k l} \mathrm{H}^{\left(10_{r}\right) m}+\cdots\right] } \\
&=i \sum_{r=1}^{2} f^{10_{r}} {\left[2 \sqrt{2}\left(-\mathrm{M}^{a \alpha} \mathrm{M}_{\alpha}+\mathrm{M}^{b a} \mathrm{M}_{b}+\cdots\right) \mathrm{H}_{a}^{\left(10_{r}\right)}\right.} \\
&\left.+\frac{1}{2 \sqrt{2}}\left(-4 \epsilon_{\alpha \beta \gamma a b} \mathrm{M}^{\alpha \beta} \mathrm{M}^{a \gamma} \mathbf{H}^{\left(10_{r}\right) b}+\cdots\right)+\cdots\right] \\
&=i \sum_{r=1}^{2} f^{10_{r}}\left[2 \sqrt{2}\left(-\mathbf{Q}^{a \alpha} \mathbf{D}_{\alpha}^{\mathrm{c}}+\epsilon^{a b} \mathbf{E}^{\mathrm{c}} \mathbf{L}_{b}\right) \mathbf{H}_{a}^{\left(10_{r}\right)}-\frac{8}{2 \sqrt{2}} \epsilon_{a b} \mathbf{U}_{\alpha}^{\mathrm{c}} \mathbf{Q}^{a \alpha} \mathbf{H}^{\left(10_{r}\right) b}+\cdots\right] .
\end{aligned}
$$

Yukawa Lagrangian of the MSSM is given by

$$
\mathcal{L}_{\text {Yuk }}=+h_{\tau}^{0} \epsilon^{a b} \mathbf{H}_{\mathbf{d} a} \mathbf{L}_{b} \mathbf{E}^{\mathrm{c}}-h_{b}^{0} \mathbf{H}_{\mathbf{d} a} \mathbf{Q}^{a \alpha} \mathbf{D}_{\alpha}^{\mathrm{c}}-h_{t}^{0} \epsilon_{a b} \mathbf{H}_{\mathbf{u}}{ }^{a} \mathbf{Q}^{b \alpha} \mathbf{U}_{\alpha}^{\mathrm{c}}+\text { h.c. }
$$

while the third generation Yukawas arising from eq. (3.2) are given by

$$
\begin{aligned}
& h_{\tau}^{0}=i 2 \sqrt{2} \sum_{r=1}^{2} f^{10_{r}} V_{d_{r 1}}, \\
& h_{b}^{0}=-i 2 \sqrt{2} \sum_{r=1}^{2} f^{10_{r}} V_{d_{r 1}}, \\
& h_{t}^{0}=-i 2 \sqrt{2} \sum_{r=1}^{2} f^{10_{r}} U_{d_{r 1}},
\end{aligned}
$$

where $U_{d r 1}$ and $V_{d r 1}$ are defined by eq. (2.5) and evaluated numerically in tables 2 and 3. 


\section{Corrections to Yukawa couplings from higher dimensional operators}

In addition to the cubic interactions in this model, contributions to the quark and lepton masses arise from quartic interactions suppressed by a heavy mass $M_{c}$ of the type (matter)(matter)(light Higgs) (heavy Higgs) $/ M$ when the heavy Higgs fields develop a large VEV. We assume that such higher dimensional operators arise from a high scale above the grand unification scale, possibly at the string scale, and are suppressed by a heavy mass $M_{c}$ larger than the grand unification mass. In the following we consider contributions arising from the higher dimensional operators $W_{4}$ involving matter fields and the Higgs fields consisting of the 10-plets, 120-plet and the 210-plet of Higgs so that

$$
W_{4}=W_{4}^{(1)}+W_{4}^{(2)}+W_{4}^{(3)},
$$

where

$$
\begin{aligned}
& W_{4}^{(1)}=-\frac{f^{(1)}}{5 ! M_{c}} b_{r}\left\langle\Psi_{(+)}^{*}\left|B \Gamma_{[\lambda} \Gamma_{\mu} \Gamma_{\nu} \Gamma_{\rho} \Gamma_{\sigma]}\right| \Psi_{(+)}\right\rangle\left[{ }^{r} \Omega_{\lambda} \Phi_{\mu \nu \rho \sigma}-{ }^{r} \Omega_{\mu} \Phi_{\lambda \nu \rho \sigma}+{ }^{r} \Omega_{\nu} \Phi_{\lambda \mu \rho \sigma}\right. \\
& \left.-{ }^{r} \Omega_{\rho} \Phi_{\lambda \mu \nu \sigma}+{ }^{r} \Omega_{\sigma} \Phi_{\lambda \mu \nu \rho}\right] \text {, } \\
& W_{4}^{(2)}=-\frac{f^{(2)}}{5 ! M_{c}}\left\langle\Psi_{(+)}^{*}\left|B \Gamma_{[\lambda} \Gamma_{\mu} \Gamma_{\nu} \Gamma_{\rho} \Gamma_{\sigma]}\right| \Psi_{(+)}\right\rangle\left[\Sigma_{\lambda \alpha \beta} \Phi_{\gamma \rho \sigma \lambda}-\Sigma_{\lambda \alpha \gamma} \Phi_{\beta \rho \sigma \lambda}+\Sigma_{\lambda \alpha \rho} \Phi_{\beta \gamma \sigma \lambda}\right. \\
& -\Sigma_{\lambda \alpha \sigma} \Phi_{\beta \gamma \rho \lambda}-\Sigma_{\lambda \gamma \beta} \Phi_{\alpha \rho \sigma \lambda}+\Sigma_{\lambda \rho \beta} \Phi_{\alpha \gamma \sigma \lambda} \\
& -\Sigma_{\lambda \sigma \beta} \Phi_{\alpha \gamma \rho \lambda}-\Sigma_{\lambda \gamma \rho} \Phi_{\beta \alpha \sigma \lambda}+\Sigma_{\lambda \gamma \sigma} \Phi_{\beta \alpha \rho \lambda} \\
& \left.-\Sigma_{\lambda \rho \sigma} \Phi_{\beta \alpha \gamma \lambda}\right] \text {, } \\
& W_{4}^{(3)}=\frac{f^{(3)}}{M_{c}}\left\langle\Psi_{(+)}^{*}\left|B \Gamma_{\mu}\right| \Psi_{(+)}\right\rangle \Sigma_{\rho \sigma \lambda} \Phi_{\rho \sigma \lambda \mu}
\end{aligned}
$$

The contribution from eq. (4.2) is computed in appendix D and the contribution from this term to the third generation Yukawas is given by

$$
\begin{aligned}
& \delta h_{t}^{(1)}=\frac{i f^{(1)}}{60 \sqrt{2} M_{c}}\left(\sum_{r=1}^{2} b_{r} U_{d_{r 1}}\right)\left[\frac{5 \sqrt{3}}{2} \mathcal{V}_{75_{210}}-4 \sqrt{15} \mathcal{V}_{24_{210}}-8 \sqrt{15} \mathcal{V}_{1_{210}}\right], \\
& \delta h_{b}^{(1)}=\frac{i f^{(1)}}{60 \sqrt{2} M_{c}}\left(\sum_{r=1}^{2} b_{r} V_{d_{r 1}}\right)\left[\frac{\sqrt{20}}{3} \mathcal{V}_{75_{210}}-20 \sqrt{\frac{5}{3}} \mathcal{V}_{24_{210}}\right], \\
& \delta h_{\tau}^{(1)}=\frac{i f^{(1)}}{60 \sqrt{2} M_{c}}\left(\sum_{r=1}^{2} b_{r} V_{d_{r 1}}\right)\left[20 \sqrt{3} \mathcal{V}_{75_{210}}-20 \sqrt{15} \mathcal{V}_{24_{210}}\right],
\end{aligned}
$$

where the VEVs of the Standard Model singlets denoted by $\mathcal{V}$ 's are defined in eq. (A.6). We refer to appendix D for further details of the computation. The contribution from eq. (4.3) is computed in appendix D and they produce the following contributions to the 
third generation Yukawas

$$
\begin{aligned}
& \delta h_{t}^{(2)}=-\frac{i f^{(2)}}{120 M_{c}}\left[\frac{10}{3} \sqrt{\frac{2}{3}} \mathcal{V}_{75_{210}} U_{d_{61}}+\frac{5}{3} \sqrt{\frac{10}{3}} \mathcal{V}_{24_{210}} U_{d_{61}}+6 \sqrt{5} \mathcal{V}_{24_{210}} U_{d_{31}}\right. \\
& \left.-8 \sqrt{5} \mathcal{V}_{1_{210}} U_{d_{31}}\right] \\
& \delta h_{b}^{(2)}=-\frac{i f^{(2)}}{120 M_{c}}\left[-\frac{20}{3} \sqrt{\frac{2}{3}} \mathcal{V}_{75_{210}} V_{d_{61}}-\frac{20}{3} \mathcal{V}_{75_{210}} V_{d_{31}}-\frac{1}{3} \sqrt{\frac{10}{3}} \mathcal{V}_{24_{210}} V_{d_{61}}\right. \\
& \left.-\frac{10 \sqrt{5}}{3} \mathcal{V}_{24_{210}} V_{d_{31}}-4 \sqrt{\frac{10}{3}} \mathcal{V}_{1_{210}} V_{d_{61}}\right] \\
& \delta h_{\tau}^{(2)}=-\frac{i f^{(2)}}{120 M_{c}}\left[-20 \sqrt{\frac{2}{3}} \mathcal{V}_{75_{210}} V_{d_{61}}-20 \mathcal{V}_{75_{210}} V_{d_{31}}-\sqrt{\frac{10}{3}} \mathcal{V}_{24_{210}} V_{d_{61}}\right. \\
& \left.-10 \sqrt{5} \mathcal{V}_{24_{210}} V_{d_{31}}-4 \sqrt{30} \mathcal{V}_{1_{210}} V_{d_{61}}\right]
\end{aligned}
$$

Finally we compute the contribution arising from eq. (4.4). From the analysis of appendix $\mathrm{D}$ we find

$$
\begin{aligned}
& \delta h_{t}^{(3)}=-\frac{3 i}{8} \frac{f^{(3)}}{M_{c}}\left[\frac{2}{3} \sqrt{\frac{2}{3}} \mathcal{V}_{75_{210}} U_{d_{61}}+\frac{1}{3} \sqrt{\frac{10}{3}} \mathcal{V}_{24_{210}} U_{d_{61}}-\frac{2}{\sqrt{5}} \mathcal{V}_{24_{210}} U_{d_{31}}\right. \\
& \left.+\frac{8}{3 \sqrt{5}} \mathcal{V}_{1_{210}} U_{d_{31}}\right] \\
& \delta h_{b}^{(3)}=-\frac{3 i}{8} \frac{f^{(3)}}{M_{c}}\left[\frac{2}{3} \sqrt{\frac{2}{3}} \mathcal{V}_{75_{210}} V_{d_{61}}+\frac{1}{3} \sqrt{\frac{10}{3}} \mathcal{V}_{24_{210}} V_{d_{61}}-\frac{2}{\sqrt{5}} \mathcal{V}_{24_{210}} V_{d_{31}}\right. \\
& \left.+\frac{8}{3 \sqrt{5}} \mathcal{V}_{1_{210}} V_{d_{31}}\right] \\
& \delta h_{\tau}^{(3)}=\frac{3 i}{8} \frac{f^{(3)}}{M_{c}}\left[\frac{2}{3} \sqrt{\frac{2}{3}} \mathcal{V}_{75_{210}} V_{d_{61}}+\frac{1}{3} \sqrt{\frac{10}{3}} \mathcal{V}_{24210} V_{d_{61}}-\frac{2}{\sqrt{5}} \mathcal{V}_{24210} V_{d_{31}}\right. \\
& \left.+\frac{8}{3 \sqrt{5}} \mathcal{V}_{1_{210}} V_{d_{31}}\right]
\end{aligned}
$$

The total Yukawas are the sum of the contributions from the cubic and from the quartic terms at the GUT scale. Thus we have

$h_{t}=h_{t}^{0}+\delta h_{t}^{(1)}+\delta h_{t}^{(2)}+\delta h_{t}^{(3)}, h_{b}=h_{b}^{0}+\delta h_{b}^{(1)}+\delta h_{b}^{(2)}+\delta h_{b}^{(3)}, h_{\tau}=h_{\tau}^{0}+\delta h_{\tau}^{(1)}+\delta h_{\tau}^{(2)}+\delta h_{\tau}^{(3)}$.

In the renormalization group (RG) evolution, eq. (4.14) acts as the boundary condition which produces the effective Yukawas at the electroweak scale $Q$ so that at this scale the top, bottom, and tau lepton masses are related to the effective Yukawa couplings so that

$$
m_{t}(Q)=\frac{h_{t}(Q) v \sin \beta}{\sqrt{2}}, \quad m_{b}(Q)=\frac{h_{b}(Q) v \cos \beta}{\sqrt{2}}, \quad m_{\tau}(Q)=\frac{h_{\tau}(Q) v \cos \beta}{\sqrt{2}},
$$

where we used the relations $\left\langle H_{d}\right\rangle=\frac{v}{\sqrt{2}} \cos \beta$ and $\left\langle H_{u}\right\rangle=\frac{v}{\sqrt{2}} \sin \beta$, and where $v=246 \mathrm{GeV}$. 


\section{Analysis of model implications}

In this section we discuss the implications of the model discussed above. Here we will give numerical computations of the cubic and the quartic interactions to the Yukawa couplings of the third generation of quarks and of the charged lepton and show that significant deviations exist at the GUT scale from the universal value of the top, bottom, and the tau Yukawa couplings predicted by a single 10-plet of $\mathrm{SO}(10)$ mode. These important corrections allow one to do two things: first unlike the case of a single 10-plet of $\mathrm{SO}(10)$ the presence of two 10 -plets already give unequal Yukawas for the top and the bottom quarks. This already implies that a $\tan \beta$ as large as 50 is no longer needed for consistency with the experimental data on the top and bottom quark masses. In addition one finds that in this class of models the quartic couplings typically contribute substantial amounts to the Yukawa couplings at the GUT scale because $\left.\langle\Phi\rangle / M_{c}\right)$ is non-negligible and thus quartic interactions give significant contributions of size comparable to those of the cubic ones. Further, because of the experimental discovery that the Higgs boson mass at $125 \mathrm{GeV}[32,33]$ requires the size of weak scale supersymmetry to lie in the $\mathrm{TeV}$ region, the sparticle spectrum for the scalars is typically in the $\mathrm{TeV}$ region, and the current experimental limits on the gluino mass also lie in the $\mathrm{TeV}$ region. The $\mathrm{RG}$ evolution of the Yukawas is sensitive to the sparticle spectrum and thus both the GUT boundary conditions and the sparticle spectrum enter in a significant way in achieving consistency with the data on the third generation masses for which currently the experimental limits are [34]

$$
\begin{aligned}
m_{t}(\text { pole }) & =172.25 \pm 0.08 \pm 0.62 \mathrm{GeV} \\
m_{b}\left(\bar{m}_{b}\right) & =4.18_{-0.03}^{+0.04} \mathrm{GeV} \\
m_{\tau}(\text { pole }) & =1.77686 \pm 0.00012 \mathrm{GeV}
\end{aligned}
$$

Thus in this analysis we give a specific set of benchmarks where consistency with the data of eq. (5.1) is achieved with Yukawa couplings at the GUT scale including contributions from the cubic and the quartic terms in matter-Higgs interactions. We follow this up by a collider analysis of some of the benchmarks for some of the sparticle spectrum that would be accessible at HL-LHC and HE-LHC. Further details of the analysis are as follows. For the high scale parameters of the Higgs sector, i.e., $M_{c}, M^{210}, \eta$ and $\lambda$, we take the ranges $0.1 \leq \eta, \lambda \leq 2.0,2 \times 10^{17} \leq M_{c} \leq 8.5 \times 10^{17}$, and $1 \times 10^{15} \leq M^{210} \leq 2.5 \times 10^{16}$. We assume that the $\mathrm{SO}(10)$ is broken near the scale $M^{126}=M_{c}$ to $\mathrm{SU}(5)$ while $\mathrm{SU}(5)$ is broken after the 210-plet of Higgs develops a VEV. Ten representative benchmarks are chosen from this set. We then look at the spontaneous breaking of the GUT symmetry which breaks the $\mathrm{SO}(10)$ gauge symmetry to the gauge symmetry of the standard model. The VEVs that enter are $\mathcal{V}_{1_{210}}, \mathcal{V}_{24_{210}}, \mathcal{V}_{75_{210}}$, and $\mathcal{V}_{1_{126}}$. Details of the spontaneous breaking of the GUT symmetry is given in appendix B. The numerical analysis of the VEVs for the benchmarks is presented in table 1.

To generate a pair of light Higgs doublets needed for electroweak symmetry breaking, we use the superpotential of eq. (2.2), and the results of eqs. (B.1)-(B.6). Here as discussed earlier the number of Higgs doublet pairs are seven which produce a $7 \times 7$ Higgs doublet mass 


\begin{tabular}{|l|cccccccc|}
\hline Model & $\eta$ & $\lambda$ & $M^{126}$ & $M^{210}$ & $\mathcal{V}_{1_{210}}$ & $\mathcal{V}_{24_{210}}$ & $\mathcal{V}_{75_{210}}$ & $\mathcal{V}_{1_{126}}$ \\
\hline (a) & 4.33 & 2.39 & $8.07 \times 10^{17}$ & $4.72 \times 10^{15}$ & $1.44 \times 10^{18}$ & $(-2.86-\imath 0.67) \times 10^{18}$ & $(-6.51-\imath 0.60) \times 10^{18}$ & $(3.49+\imath 0.24 i) \times 10^{17}$ \\
(b) & 1.57 & 2.75 & $2.74 \times 10^{17}$ & $4.48 \times 10^{15}$ & $1.36 \times 10^{18}$ & $-2.64 \times 10^{18}$ & $-1.44 \times 10^{18}$ & $\imath 2.73 \times 10^{18}$ \\
(c) & 1.54 & 0.33 & $6.55 \times 10^{17}$ & $2.43 \times 10^{16}$ & $3.30 \times 10^{18}$ & $-3.69 \times 10^{18}$ & $-1.30 \times 10^{18}$ & $\imath 1.95 \times 10^{18}$ \\
(d) & 3.39 & 4.46 & $7.87 \times 10^{17}$ & $1.26 \times 10^{16}$ & $1.80 \times 10^{18}$ & $-3.47 \times 10^{18}$ & $-1.88 \times 10^{18}$ & $\imath 3.12 \times 10^{18}$ \\
(e) & 1.88 & 0.43 & $7.58 \times 10^{16}$ & $2.27 \times 10^{16}$ & $3.13 \times 10^{17}$ & $3.52 \times 10^{17}$ & $-2.89 \times 10^{17}$ & $\imath 9.49 \times 10^{16}$ \\
(f) & 2.59 & 1.52 & $4.15 \times 10^{17}$ & $1.14 \times 10^{16}$ & $1.24 \times 10^{18}$ & $-2.15 \times 10^{18}$ & $-1.07 \times 10^{18}$ & $\imath 1.38 \times 10^{18}$ \\
(g) & 2.57 & 1.71 & $2.10 \times 10^{17}$ & $3.35 \times 10^{15}$ & $6.33 \times 10^{17}$ & $(-1.23-\imath 0.44) \times 10^{18}$ & $(-2.88-\imath 0.41) \times 10^{18}$ & $(2.52+\imath 0.25) \times 10^{17}$ \\
(h) & 2.77 & 2.95 & $5.84 \times 10^{17}$ & $2.16 \times 10^{16}$ & $1.63 \times 10^{18}$ & $-2.94 \times 10^{18}$ & $-1.51 \times 10^{18}$ & $\imath 2.48 \times 10^{18}$ \\
(i) & 2.98 & 2.33 & $6.66 \times 10^{17}$ & $2.35 \times 10^{16}$ & $1.73 \times 10^{18}$ & $-3.02 \times 10^{18}$ & $-1.51 \times 10^{18}$ & $\imath 2.23 \times 10^{18}$ \\
(j) & 1.40 & 0.12 & $2.31 \times 10^{17}$ & $2.32 \times 10^{16}$ & $1.28 \times 10^{18}$ & $1.26 \times 10^{18}$ & $-8.24 \times 10^{17}$ & $\imath 2.84 \times 10^{17}$ \\
\hline
\end{tabular}

Table 1. A numerical estimate of the VEVs of the Standard Model singlets in 210, 126 and $\overline{126}$ plets arising in the spontaneous breaking of the $\mathrm{SO}(10)$ GUT gauge symmetry under the assumption $\mathcal{V}_{1_{126}}=\mathcal{V}_{1_{\overline{126}}}$. All VEVs and masses are in GeV.

\begin{tabular}{|l|ccccccccc|}
\hline Model & $a$ & $b_{1}$ & $b_{2}$ & $c$ & $\bar{c}$ & $U_{d_{11}}$ & $U_{d_{21}}$ & $U_{d_{31}}$ & $U_{d_{61}}$ \\
\hline (a) & 4.09 & 3.06 & 2.92 & 0.04 & 3.89 & $-0.105-\imath 0.092$ & $0.114+\imath 0.093$ & $0.296-\imath 0.346$ & $-0.669+\imath 0.551$ \\
(b) & 0.39 & 1.07 & 0.21 & 0.99 & 1.89 & $-0.122+\imath 0.006$ & $0.924-\imath 0.049$ & $0.078-\imath 0.004$ & $-0.349+\imath 0.018$ \\
(c) & 1.19 & 0.59 & 0.60 & 0.45 & 1.59 & $0.185+\imath 0.045$ & $-0.239-\imath 0.058$ & $-0.093-\imath 0.023$ & $0.919+\imath 0.223$ \\
(d) & 3.63 & 2.55 & 3.86 & 0.09 & 3.65 & $-0.071-\imath 0.009$ & $0.051+\imath 0.006$ & $0.212+\imath 0.026$ & $-0.966-\imath 0.118$ \\
(e) & 0.54 & 1.38 & 0.12 & 0.34 & 0.86 & $0.022-\imath 0.003$ & $-0.236+\imath 0.036$ & $0.002-\imath 0.000$ & $-0.960+\imath 0.146$ \\
(f) & 2.72 & 1.26 & 0.55 & 0.32 & 0.60 & $-0.018+\imath 0.001$ & $0.127-\imath 0.006$ & $0.182-\imath 0.008$ & $-0.974+\imath 0.045$ \\
(g) & 2.99 & 2.23 & 1.82 & 0.49 & 2.43 & $-0.081-\imath 0.141$ & $0.192+\imath 0.139$ & $0.421-\imath 0.155$ & $-0.843+\imath 0.075$ \\
(h) & 0.88 & 0.16 & 2.68 & 0.90 & 1.16 & $-0.101+\imath 0.004$ & $0.059-\imath 0.003$ & $0.193-\imath 0.008$ & $-0.973+\imath 0.043$ \\
(i) & 1.64 & 2.59 & 0.88 & 0.35 & 0.43 & $-0.021+\imath 0.000$ & $0.120-\imath 0.002$ & $0.184-\imath 0.003$ & $-0.975+\imath 0.018$ \\
(j) & 0.20 & 1.29 & 1.06 & 0.18 & 1.41 & $-0.213-\imath 0.269$ & $0.258+\imath 0.326$ & $0.005+\imath 0.006$ & $0.523+\imath 0.660$ \\
\hline
\end{tabular}

Table 2. A numerical estimate of the elements of the down Higgs zero mode eigenvector using the analysis of table 1 and the couplings of eq. (2.2).

matrix $M_{d}$ given in appendix $\mathrm{C}$ which we diagonalize to recover a light Higgs doublet. The matrix $M_{d}$ is not symmetric and needs to be diagonalized by a biunitary transformation so that

$$
U_{d}^{\dagger} M_{d} V_{d}=M_{d}^{\text {diag }}=\left(0, m_{d_{2}}, m_{d_{3}}, \cdots, m_{d_{7}}\right) .
$$

The massless mode is identified as the Higgs doublet pair that enters in the electroweak symmetry breaking. The Higgs doublets in this pair do not involve components from $126+\overline{126}+210$ heavy Higgs and have components only from $2 \times 10+120$ light Higgs. For that reason the non-vanishing parts of $U_{d}$ are the components $U_{d_{11}}, U_{d_{21}}, U_{d_{31}}, U_{d_{61}}$ and similarly for $V_{d}$. These are recorded in table 2 and table 3 . Here the parameters $a, b_{1,2}, c$ and $\bar{c}$ are as defined in appendix $\mathrm{C}$ and are taken to be in the range $0.1-2.0$.

Next we give a computation of the Yukawa couplings at the GUT scale. As discussed in section 4, contributions to the Yukawa couplings arise from cubic interactions of eq. (3.2) and from quartic interactions of eqs. (4.2)-(4.4). The couplings that enter here are: $f^{10_{1}}, f^{10_{2}}, f^{(1)}, f^{(2)}$ and $f^{(3)}$. Using the values of these parameters given in table 4 , 


\begin{tabular}{|l|ccccccccc|}
\hline Model & $a$ & $b_{1}$ & $b_{2}$ & $c$ & $\bar{c}$ & $V_{d_{11}}$ & $V_{d_{21}}$ & $V_{d_{31}}$ & $V_{d_{61}}$ \\
\hline (a) & 4.09 & 3.06 & 2.92 & 0.04 & 3.89 & $-0.320+\imath 0.000$ & $0.336-\imath 0.002$ & $-0.412+\imath 0.000$ & $0.772-\imath 0.136$ \\
(b) & 0.39 & 1.07 & 0.21 & 0.99 & 1.89 & -0.175 & 0.963 & -0.045 & 0.201 \\
(c) & 1.19 & 0.59 & 0.60 & 0.45 & 1.59 & -0.106 & 0.215 & -0.098 & 0.966 \\
(d) & 3.63 & 2.55 & 3.86 & 0.09 & 3.65 & 0.172 & -0.115 & 0.210 & -0.955 \\
(e) & 0.54 & 1.38 & 0.12 & 0.34 & 0.86 & -0.003 & -0.006 & -0.002 & 1.000 \\
(f) & 2.72 & 1.26 & 0.55 & 0.32 & 0.60 & 0.033 & -0.123 & 0.183 & -0.975 \\
(g) & 2.99 & 2.23 & 1.82 & 0.49 & 2.43 & $0.278+\imath 0.000$ & $-0.350+\imath 0.050$ & $0.419-\imath 0.000$ & $-0.762+\imath 0.205$ \\
(h) & 0.88 & 0.16 & 2.68 & 0.90 & 1.16 & 0.206 & -0.037 & 0.190 & -0.959 \\
(i) & 1.64 & 2.59 & 0.88 & 0.35 & 0.43 & -0.039 & 0.146 & -0.183 & 0.971 \\
(j) & 0.20 & 1.29 & 1.06 & 0.18 & 1.41 & 0.051 & -0.071 & 0.009 & 0.996 \\
\hline
\end{tabular}

Table 3. A numerical estimate of the elements of the up Higgs zero mode eigenvector using the analysis of table 1 and the couplings of eq. (2.2).

\begin{tabular}{|l|cccc|}
\hline Model & $f^{(1)}$ & $f^{(2)}$ & $f^{(3)}$ & $f^{10_{r}}$ \\
\hline (a) & 0.54 & 0.02 & 0.14 & $(0.36,0.78)$ \\
(b) & 0.08 & 0.08 & 0.09 & $(1.49,0.33)$ \\
(c) & 0.16 & 0.07 & 0.14 & $(0.55,0.71)$ \\
(d) & 0.08 & 0.01 & 0.18 & $(0.76,2.18)$ \\
$(\mathrm{e})$ & 0.54 & 0.18 & 0.03 & $(1.81,0.89)$ \\
(f) & 0.08 & 0.03 & 0.08 & $(1.96,1.23)$ \\
(g) & 0.50 & 0.25 & 0.05 & $(0.48,0.62)$ \\
(h) & 0.27 & 0.24 & 0.07 & $(0.24,2.42)$ \\
$(\mathrm{i})$ & 0.10 & 0.04 & 0.10 & $(1.83,1.16)$ \\
$(\mathrm{j})$ & 0.09 & 0.02 & 0.03 & $(0.84,0.25)$ \\
\hline
\end{tabular}

Table 4. The GUT scale parameters in the cubic and quartic superpotentials $W_{3}, W_{4}^{(1)}, W_{4}^{(2)}$ and $W_{4}^{(3)}$ for the model points $(\mathrm{a})-(\mathrm{j})$. The masses are in $\mathrm{GeV}$.

we exhibit in table 5 the contribution to the Yukawa couplings from the cubic interactions, from the quartic interactions, and their sum. Table 5 defines the Yukawa couplings for the top and the bottom quarks, and for the tau lepton at the GUT scale. To evolve the Yukawas from the GUT scale to the electroweak scale we use RG equations (RGE) within the supergravity (SUGRA) model [35-38]. The running of the RGEs is implemented with the help of SPheno-4 -0.4 [39, 40] which uses two-loop MSSM RGEs and three-loop standard model (SM) RGEs and takes into account SUSY threshold effects at the one-loop level. The larger SUSY scale makes it necessary to employ a two-scale matching condition at the electroweak and SUSY scales [41] thereby improving the calculations of the Higgs boson mass and of the sparticle spectrum. The bottom quark mass and $\alpha_{S}$ (the fine structure constant for the $\left.\mathrm{SU}(3)_{C}\right)$ are run up to the scale of the $Z$ boson mass, $M_{Z}$, using four-loop RGEs in 


\begin{tabular}{|l|ccccccccc|}
\hline Model & $h_{t}^{0}$ & $h_{b}^{0}$ & $h_{\tau}^{0}$ & $\delta h_{t}^{\mathrm{GUT}}$ & $\delta h_{b}^{\mathrm{GUT}}$ & $\delta h_{\tau}^{\mathrm{GUT}}$ & $h_{t}^{\mathrm{GUT}}$ & $h_{b}^{\mathrm{GUT}}$ & $h_{\tau}^{\mathrm{GUT}}$ \\
\hline (a) & 0.181 & 0.412 & 0.412 & 0.424 & 0.385 & 0.376 & 0.519 & 0.026 & 0.037 \\
(b) & 0.354 & 0.168 & 0.168 & 0.143 & 0.091 & 0.060 & 0.507 & 0.078 & 0.108 \\
(c) & 0.197 & 0.266 & 0.266 & 0.292 & 0.329 & 0.177 & 0.494 & 0.063 & 0.089 \\
(d) & 0.163 & 0.339 & 0.339 & 0.354 & 0.349 & 0.343 & 0.513 & 0.032 & 0.043 \\
(e) & 0.489 & 0.029 & 0.029 & 0.014 & 0.023 & 0.010 & 0.498 & 0.052 & 0.072 \\
(f) & 0.344 & 0.243 & 0.243 & 0.169 & 0.175 & 0.147 & 0.507 & 0.068 & 0.097 \\
(g) & 0.234 & 0.251 & 0.251 & 0.274 & 0.314 & 0.168 & 0.498 & 0.104 & 0.151 \\
(h) & 0.334 & 0.114 & 0.114 & 0.159 & 0.216 & 0.028 & 0.505 & 0.103 & 0.143 \\
(i) & 0.289 & 0.279 & 0.279 & 0.192 & 0.198 & 0.166 & 0.495 & 0.084 & 0.113 \\
(j) & 0.520 & 0.069 & 0.069 & 0.013 & 0.007 & 0.034 & 0.496 & 0.077 & 0.104 \\
\hline
\end{tabular}

Table 5. The magnitude of the contributions to the top, bottom, and tau Yukawa couplings from cubic interactions (columns 2-4), from quartic interactions (columns 5-7) and the magnitude of their complex sum (columns 8-10) at the GUT scale for the parameter set of table 4. The Yukawa couplings are in general complex and we add the contributions of the cubic and quartic interactions as complex numbers and exhibit only their magnitudes in the table.

the $\overline{\mathrm{MS}}$ scheme while for the top quark, the evolution starts at the pole mass and the $\overline{\mathrm{MS}}$ mass is computed by running down to the $M_{Z}$ scale including two-loop QCD corrections.

The tau mass is calculated at $M_{Z}$ including one-loop electroweak corrections. The calculation of the $\overline{\mathrm{MS}}$ Yukawas at the electroweak scale involve the first matching conditions to include SM thresholds. Those couplings are then run using 3-loop SM RGEs to $M_{\text {SUSY }}$ where the second matching takes place to include SUSY thresholds at the one-loop level and a shift is made to the $\overline{\mathrm{DR}}$ scheme. The 2-loop MSSM RGEs of the $\overline{\mathrm{DR}}$ Yukawas and gauge couplings are then run to the GUT scale where the soft SUSY breaking boundary conditions are applied. Thus in addition to the GUT scale Yukawas we define the SUGRA parameters $m_{0}, A_{0}, m_{1}, m_{2}, m_{3}$ and $\tan \beta$ where $m_{0}$ is the universal scalar mass, $A_{0}$ is the universal trilinear coupling, $m_{1}, m_{2}, m_{3}$ are the $\mathrm{U}(1), \mathrm{SU}(2), \mathrm{SU}(3)$ gaugino masses all at the GUT scale and $\tan \beta=\left\langle H_{u}\right\rangle /\left\langle H_{d}\right\rangle$ where $H_{u}$ gives mass to the up quarks and $H_{d}$ gives mass to the down quarks and the charged leptons. The choice of the SUGRA parameters is constrained by the dark matter relic density for which we take eq. (5.3) to be the upper limit, the Higgs boson mass constraint, and the experimental lower limits on sparticle masses. The result of the RG analysis is shown in table 6 and here one finds that consistency with the top, bottom and tau masses along with gauge coupling unification can be achieved for values of $\tan \beta$ as low as $\tan \beta \sim 5-10$. [A $b-t-\tau$ unification with low $\tan \beta$ also occurs in unified Higgs models involving a $144+\overline{144}$ of Higgs fields [42-46].]

We note that in table 6 the non-universality of gaugino masses plays an important role in producing the compressed spectrum which is needed in part to satisfy the relic density constraint. In general the smearing of gauge couplings will also occur. However, it turns out that there is no direct relationship between the non-universality of the gaugino mass terms 


\begin{tabular}{|l|cccccc|ccc|}
\hline Model & $m_{0}$ & $A_{0}$ & $m_{1}$ & $m_{2}$ & $m_{3}$ & $\tan \beta$ & $m_{t}($ pole $)$ & $\bar{m}_{b}\left(\bar{m}_{b}\right)$ & $m_{\tau}$ (pole) \\
\hline (a) & 3051 & -10193 & 838 & 492 & 3502 & 5 & 173.9 & 4.165 & 1.77682 \\
(b) & 1096 & 4572 & 925 & 562 & 4081 & 15 & 172.4 & 4.195 & 1.77682 \\
(c) & 4127 & 3359 & 1049 & 642 & 5498 & 12 & 174.0 & 4.210 & 1.77682 \\
(d) & 1150 & -5313 & 1177 & 676 & 3423 & 6 & 172.2 & 4.210 & 1.77682 \\
(e) & 1865 & 805 & 1440 & 861 & 6929 & 10 & 174.0 & 4.150 & 1.77682 \\
(f) & 3763 & 9793 & 1748 & 996 & 4048 & 13 & 173.1 & 4.180 & 1.77682 \\
(g) & 4027 & -4880 & 1989 & 1093 & 4560 & 20 & 173.1 & 4.170 & 1.77682 \\
(h) & 1706 & -4508 & 2596 & 3219 & 1428 & 19 & 173.6 & 4.180 & 1.77682 \\
(i) & 12196 & -1035 & 3422 & 1817 & 1687 & 15 & 173.2 & 4.160 & 1.77682 \\
(j) & 1655 & -1418 & 4492 & 4807 & 2615 & 14 & 172.8 & 4.170 & 1.77682 \\
\hline
\end{tabular}

Table 6. The SUGRA parameters sets used for RG analysis where the boundary conditions for the Yukawas for the top, bottom, and the tau are taken from table 5. In the analysis the GUT scale ranges from $8.8 \times 10^{15} \mathrm{GeV}$ to $1.6 \times 10^{16} \mathrm{GeV}$.

and corrections to the gauge coupling constants. This is so because the gaugino masses arise as soft terms and in gravity mediation such terms depend on the GUT sector, the Kahler metric and the gauge kinetic function. However, in supergravity models the corrections to the gauge couplings involve a different parameter as discussed in [47] and there is no rigid connection between the two. Because of the lack of any direct connection between the corrections in the two cases, we have not included such corrections to the evolution of the gauge couplings. Further since the unification of gauge couplings works rather well in the standard MSSM/SUGRA (for a recent analysis see [48]) one can only infer that the gravitational smearing corrections to the gauge couplings at the GUT scale are small.

We discuss now briefly the mechanism at work for achieving $t-b-\tau$ unification with a low $\tan \beta$. Here there are two components at work. First we note that unlike the case of the standard model the light pair of Higgs doublets do not arise from a single 10-plet of Higgs which is the case for the standard $\mathrm{SO}(10)$ model. Rather, the light pair of Higgs doublets have components from the two 10-plets of Higgs and one 120-plet of Higgs. This leads to a splitting of the Yukawa couplings at the GUT scale even without contributions from the higher dimensional operators as can be seen from columns 2, 3 and 4 of table 5. Second, we have additional contributions from the higher dimensional operators which contribute to the Yukawas. Together, it is then possible to achieve $t-b-\tau$ unification at low values of $\tan \beta$ in certain regions of the parameter space of supergravity models. We emphasize that the analysis does not predict the existence of a low $\tan \beta$ but only points to the possibility to achieve such a unification within the missing partner $\mathrm{SO}(10)$ with a low $\tan \beta$. Specifically large values of $\tan \beta$ are not excluded but we focus in this work on low $\tan \beta$ values as those are difficult to achieve in the standard $\mathrm{SO}(10)$ model if one wants $t-b-\tau$ unification.

Some of the sparticle spectrum for each of the model points are exhibited in table 7. The sparticle spectrum of benchmarks (a)-(g) contains light electroweakinos, i.e., of mass 


\begin{tabular}{|l|ccccccc|}
\hline Model & $h^{0}$ & $\tilde{t}$ & $\tilde{g}$ & $\tilde{\tau}$ & $\tilde{\chi}_{1}^{0}$ & $\tilde{\chi}_{1}^{ \pm}$ & $\Omega h^{2}$ \\
\hline (a) & 125.3 & 4078 & 7189 & 3022 & 356.3 & 376.9 & 0.062 \\
(b) & 124.0 & 6159 & 8180 & 983 & 379.4 & 405.0 & 0.109 \\
(c) & 125.5 & 8477 & 10949 & 4069 & 435.1 & 461.8 & 0.104 \\
(d) & 124.4 & 4589 & 6937 & 1174 & 503.9 & 528.3 & 0.088 \\
(e) & 126.1 & 9929 & 13458 & 1854 & 606.5 & 633.6 & 0.103 \\
(f) & 123.5 & 6312 & 8212 & 3644 & 758.7 & 783.3 & 0.096 \\
(g) & 126.8 & 6727 & 9194 & 3868 & 881.4 & 888.4 & 0.048 \\
(h) & 125.5 & 1171 & 3118 & 1709 & 1162 & 2394 & 0.068 \\
(i) & 124.6 & 8210 & 3949 & 12011 & 1588 & 1591 & 0.109 \\
(j) & 124.0 & 3534 & 5407 & 2261 & 2032 & 2308 & 0.056 \\
\hline
\end{tabular}

Table 7. Low scale SUSY mass spectrum showing the Higgs boson, the stop, the gluino, the stau and the light electroweakino masses and the LSP relic density for the benchmarks of table 6 .

less than $1 \mathrm{TeV}$ while stops and gluinos are much heavier. Those points will be of interest in the next section where we discuss the LHC implications. The dark matter relic density is calculated using micrOMEGAs-5.0.9 [49] and we use as an upper limit the experimental value reported by the Planck collaboration [50]

$$
\left(\Omega h^{2}\right)_{\text {PLANCK }}=0.1198 \pm 0.0012 .
$$

As seen from table 7 some model points do not saturate the relic density and thus these models can accommodate more than one dark matter component, e.g., a hidden sector Dirac fermion [51-53] or an axion [54,55]. We have checked that the spin-independent protonneutralino cross-sections are very small for such model points and thus not yet excluded. As noted earlier the benchmarks of tables 1-7 are just a sample of a larger parameter space where consistency with eq. (5.1) can be achieved with a $\tan \beta$ significantly smaller than 50. This is exhibited in the right panel of figure 1 which shows a large set of model points with $\tan \beta$ in the range $5-10$ and all of the model points exhibited have $\tan \beta$ less than 20. The GUT scale splitting of the Yukawas and their evolution to the electroweak scale is exhibited graphically for models (a), (e) and (i) in figure 2. Here the left panel shows the top and bottom Yukawas while the right panel shows the bottom and tau Yukawas. The kink in the evolution of the Yukawas is due to sparticle mass threshold effects.

\subsection{Electroweakino pair production at the LHC and their decay channels}

The low energy sparticle spectrum of the benchmarks in tables 6 and 7 contain light electroweakinos (charginos and neutralinos). In this section we investigate the potential of discovering light electroweakinos with small mass splittings at the LHC. According to table 7, points (a)-(f) possess the property of a small mass splitting between the lightest chargino and the lightest neutralino (LSP). Note that the second lightest neutralino has the same mass as the lightest chargino. Points (g) and (i) have very small mass splittings 

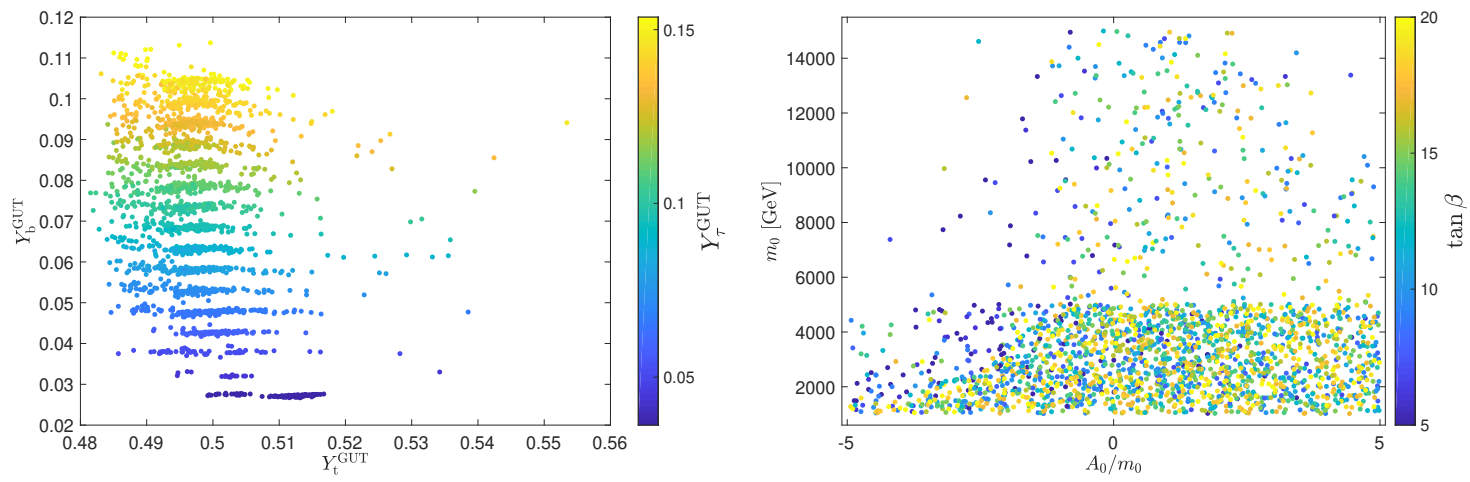

Figure 1. Left panel: a scatter plot of the top, bottom and tau GUT scale Yukawa couplings which produce the correct low scale top and bottom quark masses within a $2 \%$ theoretical uncertainty and the exact tau mass. Right panel: a scatter plot in the $m_{0}-A_{0} / m_{0}$ plane with the color axis showing $\tan \beta$.
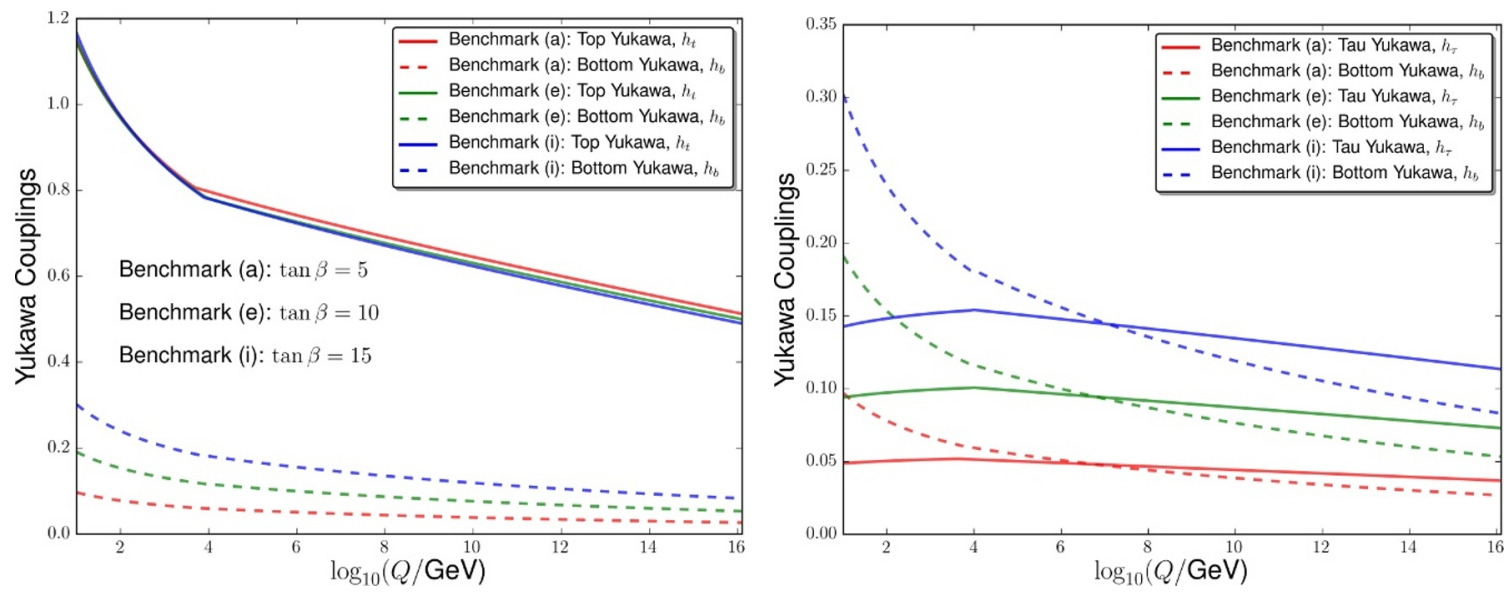

Figure 2. Left panel: the running of the top and bottom Yukawa couplings for benchmarks (a), (e) and (i). Right panel: the running of the bottom and tau Yukawas for the same benchmarks.

(less than $8 \mathrm{GeV}$ ) and require special treatment [56]. Point (h) is an example of a stop coannihilation scenario where the stop lies close in mass to the LSP while point (j) points to a stau coannihilation region. We will not consider these scenarios here (for previous works involving stop and stau coannihilation, see, e.g. $[57,58])$ but focus on the chargino coannihilation, i.e. points (a)-(f). The electroweakino mass range under study is $\sim 350 \mathrm{GeV}$ to $\sim 800 \mathrm{GeV}$ with a chargino and neutralino mass splitting of $\sim 20-27 \mathrm{GeV}$. It is worth noting that model point $(\mathrm{h})$ with a stop mass of $\sim 1.2 \mathrm{TeV}$ is within the reach of HL-LHC. The possibility of detecting electroweakinos and gluinos at HL-LHC and HE-LHC has been studied in an earlier work [48] as well as light charged and CP odd Higgs [59, 60].

Constraints on the electroweakino mass spectrum from the LHC have been taken into consideration when selecting the benchmarks under study. CMS has excluded charginos up to $230 \mathrm{GeV}$ with a mass splitting of $\sim 20 \mathrm{GeV}$ while lighter masses were excluded for larger mass splitting (down to $100 \mathrm{GeV}$ for $35 \mathrm{GeV}$ splitting) [61, 62]. More recent searches [63] 


\begin{tabular}{|l|cc|cc|ccc|}
\hline Model & \multicolumn{2}{|c|}{$\sigma_{\mathrm{NLO}+\mathrm{NNLL}}\left(p p \rightarrow \tilde{\chi}_{2}^{0} \tilde{\chi}_{1}^{ \pm}\right)$} & \multicolumn{2}{|c|}{$\sigma_{\mathrm{NLO}+\mathrm{NNLL}}\left(p p \rightarrow \tilde{\chi}_{1}^{+} \tilde{\chi}_{1}^{-}\right)$} & \multicolumn{3}{|c|}{ Branching ratios } \\
& \multicolumn{2}{|c|}{$14 \mathrm{TeV}$} & $27 \mathrm{TeV}$ & $14 \mathrm{TeV}$ & $27 \mathrm{TeV}$ & $\tilde{\chi}_{1}^{ \pm} \rightarrow \tilde{\chi}_{1}^{0} q_{i} \bar{q}_{j}$ & $\tilde{\chi}_{2}^{0} \rightarrow \tilde{\chi}_{1}^{0} \ell^{+} \ell^{-} \tilde{\chi}_{2}^{0} \rightarrow \tilde{\chi}_{1}^{0} \tau^{+} \tau^{-}$ \\
\hline$(\mathrm{a})$ & 174.3 & 540.5 & 84.9 & 270.3 & 0.67 & 0.28 & 0.14 \\
$(\mathrm{~b})$ & 129.5 & 414.3 & 62.8 & 206.8 & 0.64 & 0.29 & 0.21 \\
$(\mathrm{c})$ & 75.8 & 258.6 & 36.5 & 128.5 & 0.67 & 0.12 & 0.07 \\
$(\mathrm{~d})$ & 40.6 & 148.8 & 19.4 & 73.7 & 0.66 & 0.32 & 0.17 \\
$(\mathrm{e})$ & 18.5 & 76.7 & 8.7 & 37.7 & 0.66 & 0.33 & 0.17 \\
(f) & 6.2 & 30.8 & 2.9 & 15.0 & 0.67 & 0.07 & 0.04 \\
\hline
\end{tabular}

Table 8. The NLO+NNLL production cross-sections, in $\mathrm{fb}$, of electroweakinos: the second neutralino-chargino pair, $\tilde{\chi}_{2}^{0} \tilde{\chi}_{1}^{ \pm}$(second and third columns), and opposite sign chargino pair (fourth and fifth columns) at $\sqrt{s}=14 \mathrm{TeV}$ and at $\sqrt{s}=27 \mathrm{TeV}$ for benchmarks (a)-(f) of table 6 . Also shown are the branching ratios to quarks and leptons for the electroweakinos of the same benchmarks. Note that $q \in\{u, d, c, s\}$ and $\ell \in\{e, \mu\}$.

in the zero and one lepton channels excluded charginos up to $200 \mathrm{GeV}$ for a larger range of mass splittings, up to $50 \mathrm{GeV}$. ATLAS has put more stringent constraints on charginos and neutralinos. For the small and intermediate mass splittings [64] chargino mass up to $345 \mathrm{GeV}$ has been excluded and up to $200 \mathrm{GeV}$ also ruled out for an almost degenerate spectrum. The limit on charginos reach a mass $\sim 1.1 \mathrm{TeV}$ associated with a massless neutralino $[65,66]$. For chargino mass of more than $350 \mathrm{GeV}$, a mass splitting with the LSP of up to $50 \mathrm{GeV}$ is still allowed and that mass gap increases for heavier spectra. The benchmarks (a)-(f) are in accordance with those constraints from ATLAS and CMS.

We consider electroweakino pair production, $\tilde{\chi}_{2}^{0} \tilde{\chi}_{1}^{ \pm}$and $\tilde{\chi}_{1}^{+} \tilde{\chi}_{1}^{-}$in proton-proton collisions at $14 \mathrm{TeV}$ (HL-LHC) and $27 \mathrm{TeV}$ (HE-LHC). The NLO+NNLL production crosssections for the benchmarks (a)-(f) are calculated with Resummino-2.0.1 [67,68] using the five-flavor NNPDF23NLO PDF set. The results are shown in table 8 along with the branching ratios of $\tilde{\chi}_{2}^{0}$ and $\tilde{\chi}_{1}^{ \pm}$into the different final states of interest.

The second neutralino three-body decays into two light leptons (electrons and muons) proceed through an off-shell $Z$ and Higgs bosons. Light leptons may also come from the decay of taus. This three-body decay (shown in the last column of table 8) can also proceed via the exchange of a stau. We note that the branching ratio to two taus is particularly enhanced for benchmark (b) and this is because of a relatively light stau (983 GeV, see table 7). The three-body decay of a chargino into quarks is mediated by an off-shell $W$ boson and is the dominant decay channel as seen in table 8 .

\subsection{Signal and background simulation and event selection}

The signal which consists of electroweakino pair production can be reconstructed based on specific final states of our choice. Here we look for a pair of same flavor and opposite sign (SFOF) light leptons (electron or muons), at least two jets and a large missing transverse energy (MET). The leptons are expected to be soft as a result of the small mass splitting between the LSP and the NLSP (chargino or second neutralino). However, the lepton 
and MET systems receive a kick in momentum as they recoil against a hard initial state radiation (ISR). This ISR-assisted topology is crucial in extracting the signal from the large standard model (SM) background. The signal region (SR) will be denoted as $\mathrm{SR} 2 \ell \mathrm{N} j$ with $N \geq 2$ as the number of jets required in the final state. The dominant SM backgrounds come from diboson production, $Z / \gamma+$ jets, dilepton production from off-shell vector bosons $\left(V^{*} \rightarrow \ell \ell\right), t \bar{t}$ and $t+W / Z$. The subdominant backgrounds are Higgs production via gluon fusion $(g g F \mathrm{H})$ and vector boson fusion $(\mathrm{VBF})$. The signal and SM backgrounds are simulated at LO at $14 \mathrm{TeV}$ and $27 \mathrm{TeV}$ with MadGraph5_aMC@NLO-2.6.3 interfaced to LHAPDF [69] using the NNPDF30LO PDF set. At the generator level, up to two partons are added to the main process to produce extra jets. The parton level events are passed on to PYTHIA8 [70] for showering and hadronization using a five-flavor matching scheme in order to avoid double counting of jets. The matching/merging scale for the signal is set at $100 / 150 \mathrm{GeV}$. Additional jets from ISR and FSR (final state radiation) are allowed in order to boost the signal topology. Jets are clustered with FastJet [71] using the anti- $k_{t}$ algorithm [72] with jet radius $R=0.4$. DELPHES-3.4.2 [73] is then employed for detector simulation and event reconstruction using the beta card for HL-LHC and HE-LHC studies. The cross-sections in the resulting files are then scaled to their NLO+NNLL values for the signal samples and NLO for the SM backgrounds. The corresponding ROOT files are then analyzed and analysis cuts are implemented with the help of ROOT 6 [74].

The preselection criteria applied to the signal and background samples involve two SFOS leptons with the leading and subleading transverse momenta $p_{T}>15 \mathrm{GeV}$ for electrons and $p_{T}>10 \mathrm{GeV}$ for muons with $|\eta|<2.5$. Each event should contain at least two non-b-tagged jets with the leading $p_{T}>20 \mathrm{GeV}$ in the $|\eta|<2.4$ region. For the signal region analysis, we design a set of kinematic variables that are especially effective in reducing the SM background while retaining as much of the signal as possible. Since the signal is rich in missing transverse momentum, then a cut on $E_{T}^{\text {miss }}$ is essential in reducing the background. The SFOS dilepton invariant mass, $m_{\ell \ell}$, is calculated using the leading and subleading leptons in an event. The total transverse momentum of the dilepton system is associated with the $Z$ boson and denoted by $p_{T}^{Z}$. The dijet system, consisting of the leading and subleading jets in an event, is reconstructed and associated to a $W$ boson which is closest in $\Delta \phi$ to the $Z \rightarrow \ell \ell+\mathrm{MET}$ system. The other jets are taken to be ISR, with $p_{T}^{\mathrm{ISR}}$ denoting the vector sum of all ISR transverse momenta in an event. From these observables we determine $E_{T}^{\text {miss }} / p_{T}^{Z}$ and $\Delta \phi\left(\vec{p}_{T}^{\text {miss }}, Z\right)$ which is the opening angle between the MET and $p_{T}^{Z}$. The normalized distributions in some of those variables are shown in figure 3.

The dilepton invariant mass distribution at $14 \mathrm{TeV}$ for the benchmarks (a)-(f) is shown in the upper left panel of figure 3. Here one finds that the distributions have a peak around $20 \mathrm{GeV}$ for most points, consistent with the chargino (second neutralino)-LSP mass gap. The upper right panel shows the distribution in missing transverse energy for point (f) at $27 \mathrm{TeV}$ for an integrated luminosity of $3000 \mathrm{fb}^{-1}$. In the bottom panels we show the distributions in the ISR jet transverse momentum for point (a) at $14 \mathrm{TeV}$ (left) and at $27 \mathrm{TeV}$ (right) both for an integrated luminosity of $3000 \mathrm{fb}^{-1}$. Such distributions help design the selection criteria necessary to discriminate the signal from the SM backgrounds. The three distributions in MET and ISR jets are plotted after a selection cut on the dilepton invariant mass 

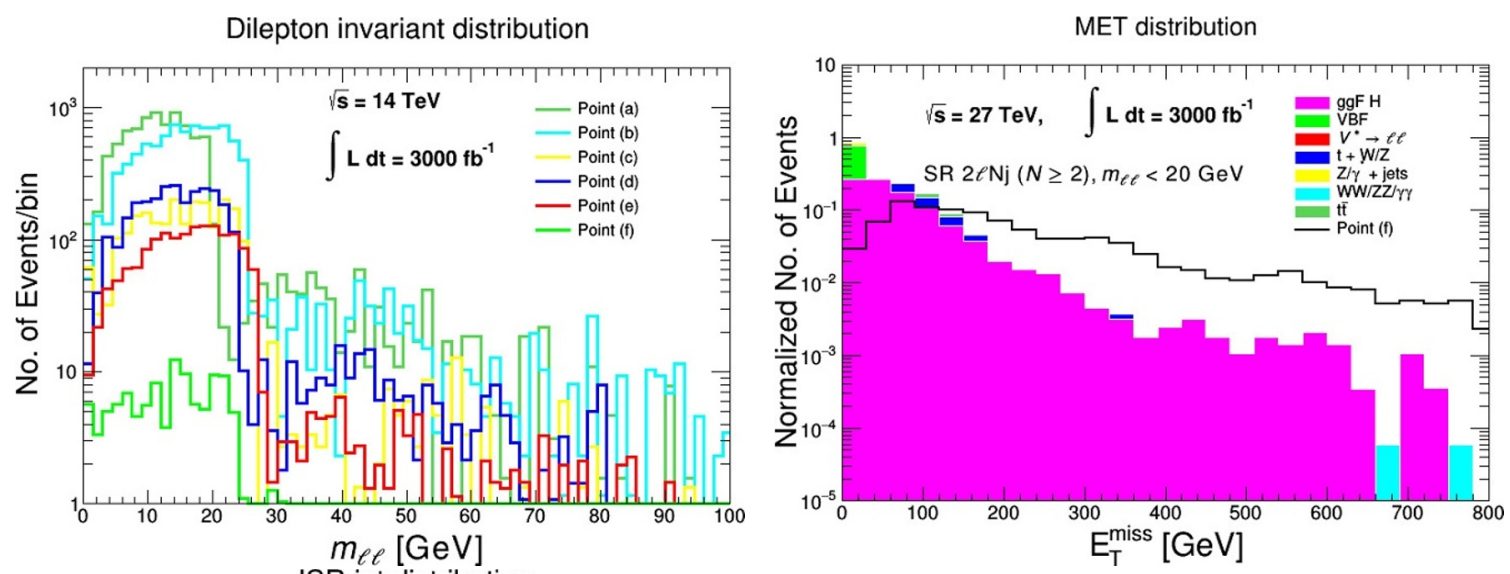

ISR jet distribution
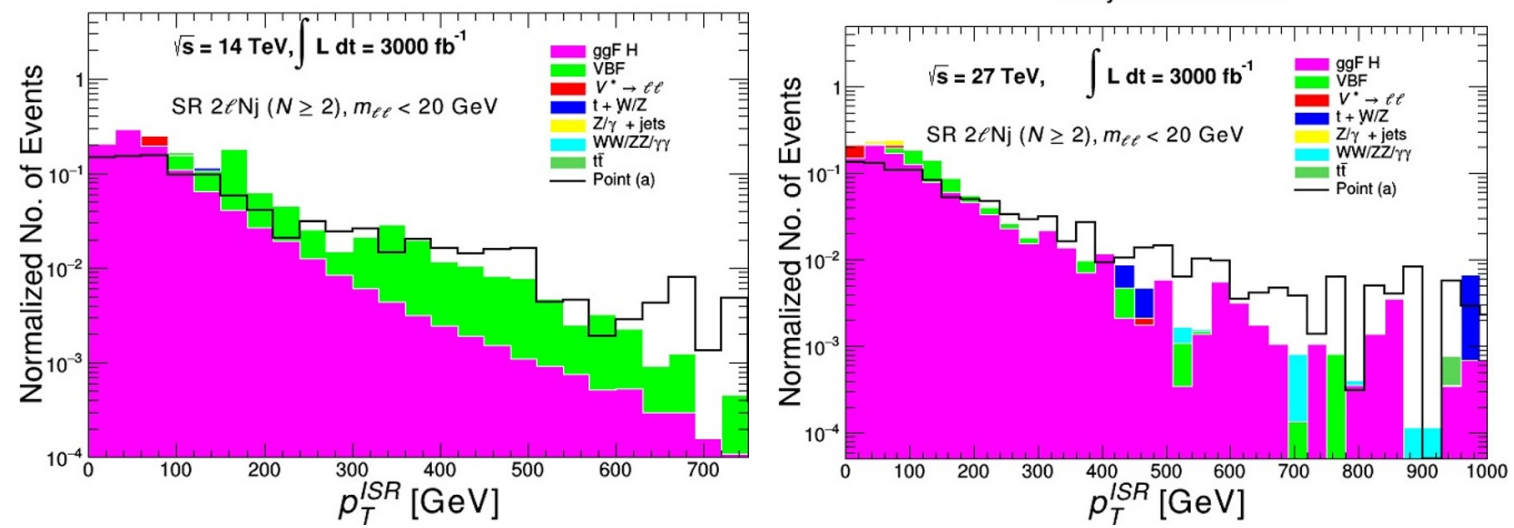

Figure 3. Top panels: an exhibition of the reconstructed dilepton invariant mass, $m_{\ell \ell}$, (left) for points (a)-(f) at $14 \mathrm{TeV}$ and the distribution in MET (right) at $27 \mathrm{TeV}$ for point (f). Bottom panels: an exhibition of the distributions in the ISR transverse momentum at $14 \mathrm{TeV}$ (left) and $27 \mathrm{TeV}$ (right) for benchmark (a).

$m_{\ell \ell}$ where events with $m_{\ell \ell}>20 \mathrm{GeV}$ are rejected. A cut around that value will remove most of the dominant backgrounds especially the $Z+$ jets which has a peak around the $Z$ boson mass. A veto on b-tagged jets will reduce the $t \bar{t}$ background and further preselection criteria on MET will reduce the rest of the SM backgrounds. The dominant background remaining is from dilepton production via off-shell vector bosons. More analysis cuts are required to reduce such a background. We summarize the preselection and selection criteria in table 9 .

\subsection{Cut implementation and the estimated integrated luminosity}

Selection criteria are optimized per mass range and for each collider, i.e. for HL-LHC and HE-LHC. Starting with HL-LHC, the two signal regions we consider are SR $2 \ell N \mathrm{j}-\mathrm{A}$ and SR $2 \ell N \mathrm{j}-\mathrm{B}$. They have the same preselection criteria but differ in terms of the analysis cuts on the variables $E_{T}^{\text {miss }}$ and $m_{\ell \ell}$ as shown in table 9 . Signal regions pertaining to HE-LHC are termed SR $2 \ell N j-C$ and SR $2 \ell N j-D$ and as HL-LHC, the only differences are in the same two variables mentioned before. For HE-LHC, harder cuts on $E_{T}^{\mathrm{miss}}, p_{T}^{\mathrm{ISR}}$ and $E_{T}^{\mathrm{miss}} / p_{T}^{Z}$ are applied. Another variable used in the analysis cuts is $\Delta \phi\left(\vec{p}_{T}^{\text {miss }}, Z\right)$ which is the opening 


\begin{tabular}{|c|c|c|c|c|}
\hline \multirow{3}{*}{ Observable } & $\mathrm{SR}-2 \ell \mathrm{Nj}-\mathrm{A}$ & 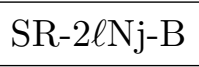 & 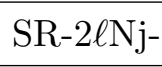 & SR- $2 \ell \mathrm{Nj}-\mathrm{D}$ \\
\hline & \multicolumn{2}{|c|}{$14 \mathrm{TeV}$} & \multicolumn{2}{|c|}{$27 \mathrm{TeV}$} \\
\hline & \multicolumn{4}{|c|}{ Preselection criteria } \\
\hline$N_{\ell}(\mathrm{SFOS})$ & \multicolumn{2}{|c|}{2} & \multicolumn{2}{|c|}{2} \\
\hline$N_{\text {jets }}^{\text {non-b-tagged }}$ & \multicolumn{2}{|c|}{$\geq 2$} & \multicolumn{2}{|c|}{$\geq 2$} \\
\hline$p_{T}^{\text {leading jet }}[\mathrm{GeV}]$ & \multicolumn{2}{|c|}{$>20$} & \multicolumn{2}{|c|}{$>20$} \\
\hline$p_{T}^{\ell}$ (electron, muon) $[\mathrm{GeV}]$ & \multicolumn{2}{|c|}{$>15,>10$} & \multicolumn{2}{|c|}{$>15,>10$} \\
\hline \multirow[t]{2}{*}{$E_{T}^{\text {miss }}[\mathrm{GeV}]$} & \multicolumn{2}{|c|}{$>90$} & \multicolumn{2}{|c|}{$>100$} \\
\hline & \multicolumn{4}{|c|}{ Analysis cuts } \\
\hline$p_{T}^{\text {ISR }}[\mathrm{GeV}]$ & $>100$ & $>100$ & $>120$ & $>120$ \\
\hline$\Delta \phi\left(\vec{p}_{T}^{\text {miss }}, Z\right)[\mathrm{rad}]$ & $<1.2$ & $<1.2$ & $<1.2$ & $<1.2$ \\
\hline$E_{T}^{\mathrm{miss}} / p_{T}^{Z}$ & $>12$ & $>15$ & $>12$ & $>25$ \\
\hline$m_{\ell \ell}[\mathrm{GeV}]$ & $<20$ & $<23$ & $<20$ & $<23$ \\
\hline
\end{tabular}

Table 9. Preselection and analysis cuts (at $14 \mathrm{TeV}$ and $27 \mathrm{TeV}$ ) applied to the signal and SM backgrounds for two signal regions targeting low and high electroweakino mass ranges.

angle between the MET and $p_{T}^{Z}$ ensuring that no jets constructed from $W$ bosons fake the dilepton system. The variable $E_{T}^{\text {miss }} / p_{T}^{Z}$ is a powerful discriminant since, unlike the backgrounds, the signal has the most MET and the softest of leptons so we expect the signal to have a larger value of this variable compared to the backgrounds. In order to design the optimal cuts on this variable, we plot the distributions in $E_{T}^{\mathrm{miss}} / p_{T}^{Z}$ for the lightest benchmark (a) and the heaviest (f) at $14 \mathrm{TeV}$ and $27 \mathrm{TeV}$. The plots are shown in figure 4 .

The top panel shows a comparison between HL-LHC (left) and HE-LHC (right) for point (a) where this benchmark can be visible at both colliders for $300 \mathrm{fb}^{-1}$ and $100 \mathrm{fb}^{-1}$ of integrated luminosity, respectively. The number of signal events in excess over the background are enough for a $5 \sigma$ discovery if a cut on the variable $E_{T}^{\text {miss }} / p_{T}^{Z}$ is made where the dashed line and arrow are located. This cut is shown in table 9 . In contrast, point (f) cannot be discovered with $\mathcal{L}=3000 \mathrm{fb}^{-1}$ at HL-LHC since the signal is completely below the background as seen in the bottom left panel of figure 4. However, one can potentially discover this model point at HE-LHC with $\sim \mathcal{L}=6500 \mathrm{fb}^{-1}$ at HE-LHC by applying a cut on $E_{T}^{\text {miss }} / p_{T}^{Z}$ where the dashed line and arrow indicate. This cut is also shown in table 9 . The estimated integrated luminosities for discovery of benchmarks (a)-(f) are shown in figure 5 .

The signal regions that give the optimal results for each of the benchmarks are shown in the plot per each collider. Starting with the lightest model point (a), a discovery at $14 \mathrm{TeV}$ can be made with only $\sim 226 \mathrm{fb}^{-1}$ which should be attainable in the upcoming Run 3 . A much smaller integrated luminosity of $\sim 62 \mathrm{fb}^{-1}$ is needed for discovery at HE-LHC. Model point (b) requires much more $\mathcal{L}$, around $1100 \mathrm{fb}^{-1}$ at HL-LHC while only $135 \mathrm{fb}^{-1}$ is needed at HE-LHC. Points (c), (d) and (e) require an integrated luminosity ranging between $1200 \mathrm{fb}^{-1}$ to $\sim 2500 \mathrm{fb}^{-1}$ for HL-LHC and $\sim 390 \mathrm{fb}^{-1}$ to $\sim 800 \mathrm{fb}^{-1}$ for HE-LHC. Point (f) 

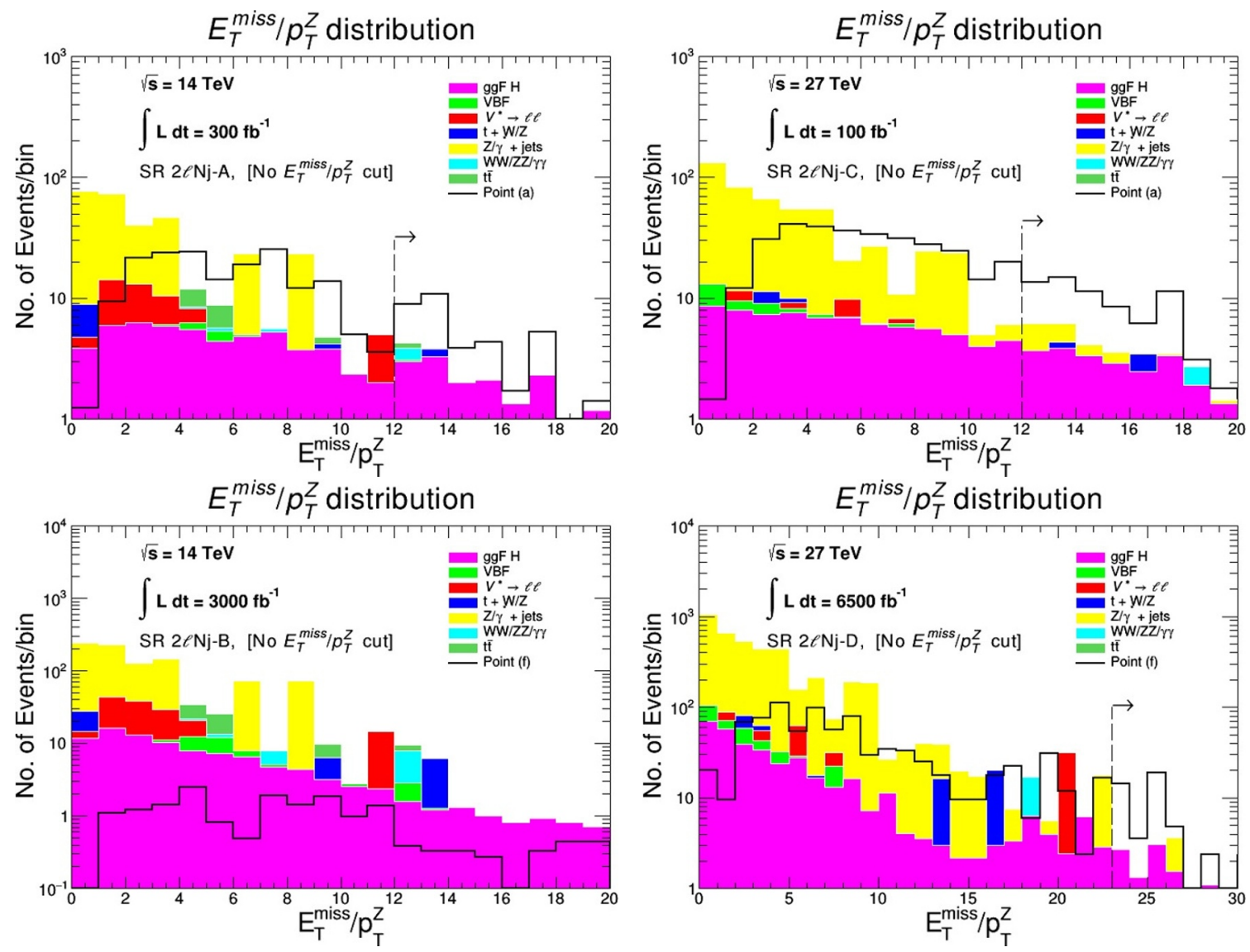

Figure 4. An exhibition of distributions in $E_{T}^{\text {miss }} / p_{T}^{Z}$ for benchmarks (a) and (f) at $14 \mathrm{TeV}$ (left panels) and $27 \mathrm{TeV}$ (right panels). The dashed line and arrow indicate the best cut on the variable.

which is only discoverable at HE-LHC require $\sim 6300 \mathrm{fb}^{-1}$. Note that despite being heavier than point (c), point (d) requires less integrated luminosity for discovery. The reason is that point (c) has a small branching ratio to dileptons (see table 8) and so the overall cross-section to the required final states is smaller. Note also that the branching ratio to leptons for point (f) is very small $(7 \%)$ compounded with the fact that it is the heaviest makes it very difficult to detect and that is why even at HE-LHC, which could potentially collect around $15 \mathrm{ab}^{-1}$ of data $[75,76]$, the required integrated luminosity is large.

\section{Conclusion}

In this work we consider a class of $\mathrm{SO}(10)$ models which lead to a pair of light Higgs doublets without the necessity of a fine tuning needed in generic grand unified models. In this class we consider a model with $126+\overline{126}+210$ of heavy Higgs and a $2 \times 10+120$ of light Higgs. The focus of this work is to show that significant contributions from the higher dimensional operators to the Yukawa couplings arise from matter-matter-HiggsHiggs interactions in the superpotential where one of the Higgs fields is light and the 


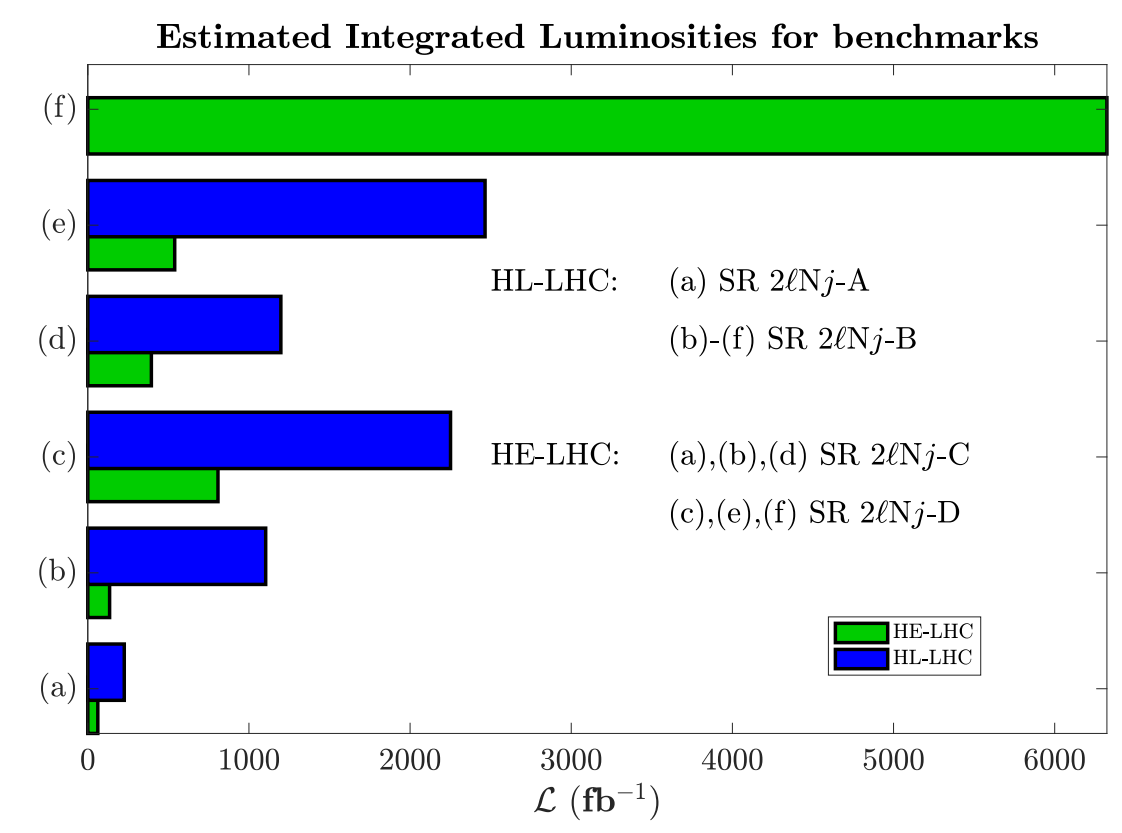

Figure 5. Estimated integrated luminosity for discovery of benchmarks (a)-(f) at HL-LHC and HE-LHC. All points except (f) are visible at HL-LHC while all points are discoverable at HE-LHC.

other heavy, even though the interactions are suppressed by a heavy mass. This occurs because the heavy field, after spontaneous symmetry breaking of the GUT symmetry, develops a VEV which is order the GUT scale which overcomes the suppression of the higher dimensional operator by the heavy mass. In this work we focused on computing the corrections to the third generation Yukawas using quartic couplings of eqs. (4.2)(4.4). The analysis shows that the contribution of the quartic terms to the Yukawas can produce substantial corrections to the GUT boundary conditions for the Yukawas. The RG evolution using the modified boundary conditions shows that a consistency with the third generation quarks and the charged leptons masses can be achieved even with a low value of $\tan \beta$, i.e., a $\tan \beta$ as low as $5-10$ consistent with gauge coupling unification. The sparticle spectrum for the models considered was investigated and it is found that the relic density as an upper limit constraint can be satisfied in three coannihilation regions that arise in the models investigated, i.e., coannihilations involving chargino-neutralino, stau-neutralino, and stop-neutralino. Further, LHC implications for some of the charginoneutralino coannihilation models was carried out for the possibility of SUSY discovery via the detection of a light chargino at HL-LHC and at a possible future collider HE-LHC at $27 \mathrm{TeV}$. It is shown that most of the models investigated can be discovered at HL-LHC using up to its optimal integrated luminosity while all of the models are discoverable at HE-LHC with a significantly smaller integrated luminosity and on a much shorter time scale. Discovery of a chargino, a stau or a stop which appear as the lightest sparticles in the analysis along with a determination of $\tan \beta$ which indicates a low value for it would 
lend support to this class of unified models. We note in passing that in the models of the type discussed the LSP can both saturate the relic density or be only a fraction of it. This implies that dark matter could be either a one component WIMP (neutralino) dark matter, or a multicomponent one where the WIMPs comprise only a fraction and the rest arises from other sources such as axions or matter from the dark sector.

\section{Acknowledgments}

The analysis presented here was done using the resources of the high-performance Cluster353 at the Advanced Scientific Computing Initiative (ASCI) and the Discovery Cluster at Northeastern University. The research of AA was supported by the BMBF under contract $05 \mathrm{H} 18 \mathrm{PMCC1}$, while the research of PN was supported in part by the NSF Grant PHY-1913328.

\section{A Notation and decomposition of $\mathrm{SU}(5)$ fields in terms of $\mathrm{SU}(3)_{C} \times$ $\mathrm{SU}(2)_{L} \times \mathrm{U}(1)_{Y}$}

$\mathrm{SO}(10)$ spinor and Higgs fields of our model in terms of SU(5) fields are

$$
\begin{aligned}
16\left[\Psi_{(+)}\right]= & 1(-5)[\mathrm{M}]+\overline{5}(3)\left[\mathrm{M}_{i}\right]+10(-1)\left[\mathrm{M}^{i j}\right], \\
10_{\mathrm{r}}\left[{ }^{r} \Omega_{\mu}\right]= & 5(2)\left[\mathrm{H}^{\left(10_{r}\right) i}\right]+\overline{5}(-2)\left[\mathrm{H}_{i}^{\left(10_{r}\right)}\right], \\
120\left[\Sigma_{\mu \nu \rho}\right]= & \left.5(2)\left[\mathrm{H}^{(120) i}\right]+\overline{5}(-2)\left[\mathrm{H}_{i}^{(120)}\right]+10(-6)+\overline{10}(6)\right]+45(2)\left[\mathrm{H}_{k}^{(120) i j}\right] \\
& +\overline{45}(-2)\left[\mathrm{H}_{i j}^{(120) k}\right], \\
\overline{126}\left[\Delta_{\mu \nu \rho \sigma \lambda}\right]= & 1(10)\left[\mathrm{H}^{(\overline{126})}\right]+5(2)\left[\mathrm{H}^{(\overline{126}) i}\right]+\overline{10}(6)+15(-6)+\overline{45}(-2)\left[\mathrm{H}_{i j}^{(\overline{126}) k}\right]+50(2), \\
210\left[\Phi_{\mu \nu \rho \sigma}\right]= & 1(0)\left[\mathrm{H}^{(210)}\right]+5(-8)\left[\mathrm{H}^{(210) i}\right]+\overline{5}(8)\left[\mathrm{H}_{i}^{(210)}\right]+10(4)+\overline{10}(-4) \\
& +24(0)\left[\mathrm{H}_{j}^{(120) i}\right]+40(-4)+\overline{40}(4)+75(0)\left[\mathrm{H}_{k l}^{(210) i j}\right],
\end{aligned}
$$

where $\mu, \nu, \rho, \sigma, \lambda=1, \ldots, 10$ and $i, j, k, l, m, n=1, \ldots, 5$ are $\mathrm{SO}(10)$ and $\mathrm{SU}(5)$ indices and $r, s=1,2$ count the number of 10 plet of $\mathrm{SO}(10)$. The identification of $\mathrm{SU}(2)$ doublets, denoted by $\mathcal{D}$ 's, contained in $5, \overline{5}, 45, \overline{45}$ of $\mathrm{SU}(5)$ are done through

$$
\begin{aligned}
& \left.\mathrm{H}^{(\#) a} \equiv{ }^{\left({ }^{*}\right.}\right)^{\mathcal{D}^{a}} ; \\
& \mathrm{H}_{a}^{(\#)} \equiv{ }^{\left(\overline{5}_{\#}\right)} \mathcal{D}_{a}, \\
& \mathrm{H}_{\beta}^{(\#) \alpha a}=\frac{1}{3} \delta_{\beta}^{\alpha}{ }^{\left(45_{\#}\right)} \mathcal{D}^{a} ; \\
& \mathrm{H}_{c}^{(\#) a b}=\delta_{c}^{b}\left(45_{\#)} \mathcal{D}^{a}-\delta_{c}^{a}\left(45_{\#}\right) \mathcal{D}^{b},\right. \\
& \mathrm{H}_{\alpha a}^{(\#) \beta}=\frac{1}{3} \delta_{\alpha}^{\beta}{ }^{\left(\overline{45}_{\#}\right)} \mathcal{D}_{a} ; \\
& \mathrm{H}_{a b}^{(\#) c}=\delta_{b}^{c}{ }^{\left(\overline{45}_{\#}\right)} \mathcal{D}_{a}-\delta_{a}^{c}{ }^{\left(\overline{45}_{\#}\right)} \mathcal{D}_{b},
\end{aligned}
$$

where $\alpha, \beta, \gamma=1,2,3$ are $\mathrm{SU}(3)$ color indices, while $a, b=4,5$ are $\mathrm{SU}(2)$ weak indices and \# refers to the 10r, 120,126, 210 fields of SO(10). Note, however, that $\mathcal{D}$ 's are unnormalized. To normalize the $\mathrm{SU}(2)$ doublets contained in the $\mathrm{SO}(10)$ tensors, we carry 
out the following field redefinition:

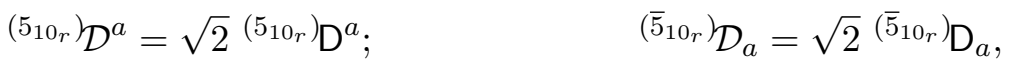

$$
\begin{aligned}
& { }^{\left(5_{120}\right)} \mathcal{D}^{a}=\frac{4}{\sqrt{3}}{ }^{\left(5_{120}\right)} \mathrm{D}^{a} ; \quad{ }^{\left(\overline{5}_{120}\right)} \mathcal{D}_{a}=\frac{4}{\sqrt{3}}{ }^{\left(\overline{5}_{120}\right)} \mathrm{D}_{a} \\
& \left.\left.{ }^{(5} \overline{\overline{126}}\right) \mathcal{D}^{a}=4 \sqrt{\frac{2}{5}}{ }^{\left({ }^{126}\right.}\right) \mathrm{D}^{a} ; \quad{ }^{\left(\overline{5}_{126}\right)} \mathcal{D}_{a}=4 \sqrt{\frac{2}{5}}{ }^{\left(\overline{5}_{126}\right)} \mathrm{D}_{a} \\
& { }^{\left(5_{210}\right)} \mathcal{D}^{a}=8 \sqrt{6}^{\left(5_{210}\right)} \mathrm{D}^{a} ; \quad{ }^{\left(\overline{5}_{210}\right)} \mathcal{D}_{a}=8 \sqrt{6}^{\left(\overline{5}_{210}\right)} \mathrm{D}_{a} \\
& { }^{\left(45_{120}\right)} \mathcal{D}^{a}=\frac{1}{\sqrt{2}}{ }^{\left(45_{120}\right)} \mathrm{D}^{a} ; \quad \quad{ }^{\left(\overline{45}_{120}\right)} \mathcal{D}_{a}=\frac{1}{\sqrt{2}}{ }^{\left(\overline{45}_{120}\right)} \mathrm{D}_{a} \\
& { }^{\left(45_{126}\right)} \mathcal{D}^{a}=\frac{1}{\sqrt{5}}{ }^{\left(45_{126}\right)} \mathrm{D}^{a} ; \quad \quad\left(\overline{(55}_{\overline{126}}\right) \mathcal{D}_{a}=\frac{1}{\sqrt{5}}{ }^{(\overline{45} \overline{126})} \mathrm{D}_{a}
\end{aligned}
$$

where D's represent the doublet fields with canonically normalized kinetic energy terms.

The identification of various $\mathrm{SU}(3)_{C} \times \mathrm{SU}(2)_{L} \times \mathrm{U}(1)_{Y}$ singlets are done through

$$
\begin{aligned}
\mathrm{H}^{(\overline{126})} & =\mathcal{S}_{1 \overline{126}} ; & \mathrm{H}^{(210)} & =\mathcal{S}_{1_{210}}, \\
\mathrm{H}_{b}^{(210) a} & =-\frac{1}{2} \delta_{b}^{a} \mathcal{S}_{24_{210}} ; & \mathrm{H}_{\beta}^{(210) \alpha} & =\frac{1}{3} \delta_{\beta}^{\alpha} \mathcal{S}_{24_{210}}, \\
\mathrm{H}_{c d}^{(210) a b} & =\frac{1}{2}\left(\delta_{c}^{a} \delta_{d}^{b}-\delta_{d}^{a} \delta_{c}^{b}\right) \mathcal{S}_{75_{210}} ; & \mathrm{H}_{\gamma \tau}^{(210) \alpha \beta} & =\frac{1}{6}\left(\delta_{\gamma}^{\alpha} \delta_{\tau}^{\beta}-\delta_{\tau}^{\alpha} \delta_{\gamma}^{\beta}\right) \mathcal{S}_{75_{210}}, \\
\mathrm{H}_{\beta b}^{(210) \alpha a} & =-\frac{1}{6} \delta_{b}^{a} \delta_{\gamma}^{\alpha} \mathcal{S}_{75_{210}} . & &
\end{aligned}
$$

$\mathcal{S}$ 's are un-normalized fields. To normalize them, we carry out the following field redefinition:

$$
\begin{array}{rlrl}
\mathcal{S}_{1_{210}} & =4 \sqrt{\frac{5}{3}} \mathbf{S}_{1_{210}} ; & \mathcal{S}_{24_{210}}=2 \sqrt{\frac{3}{5}} \mathbf{S}_{24_{210}}, \\
\mathcal{S}_{75_{210}}=\frac{1}{\sqrt{3}} \mathbf{S}_{75_{210}} ; & \mathcal{S}_{1_{126}}=\frac{2}{\sqrt{15}} \mathbf{S}_{1_{126}}, \\
\mathcal{S}_{1_{\overline{126}}}=\frac{2}{\sqrt{15}} \mathbf{S}_{1_{\overline{126}}} . &
\end{array}
$$

We will denote the VEVs of the normalized fields by $\mathcal{V}$ 's, so that

$$
\mathcal{V}_{1_{210}} \equiv\left\langle\mathbf{S}_{1_{210}}\right\rangle ; \quad \mathcal{V}_{24_{210}} \equiv\left\langle\mathbf{S}_{24_{210}}\right\rangle ; \quad \mathcal{V}_{75_{210}} \equiv\left\langle\mathbf{S}_{75_{210}}\right\rangle ; \quad \mathcal{V}_{1_{126}} \equiv\left\langle\mathbf{S}_{1_{126}}\right\rangle ; \quad \mathcal{V}_{\frac{1-126}{126}} \equiv\left\langle\mathbf{S}_{1 \frac{1}{126}}\right\rangle .
$$

The SU(5) matter fields are

$$
\begin{array}{rlrl}
\mathrm{M} & =\boldsymbol{\nu}^{\mathrm{c}} ; & \mathrm{M}_{\alpha}=\mathbf{D}_{\alpha}^{\mathrm{c}} ; & \mathrm{M}_{a}=\mathbf{L}_{a}=\left(\begin{array}{c}
\mathbf{E} \\
-\boldsymbol{\nu}
\end{array}\right) ; \\
\mathrm{M}^{a \alpha}=\mathbf{Q}^{a \alpha}=\left(\begin{array}{c}
\mathbf{U}^{\alpha} \\
\mathbf{D}^{\alpha}
\end{array}\right) ; & \mathrm{M}^{\alpha \beta}=\epsilon^{\alpha \beta \gamma} \mathbf{U}_{\gamma}^{c} ; & \mathrm{M}^{a b}=\epsilon^{a b} \mathbf{E}^{\mathrm{c}} .
\end{array}
$$

\section{$\mathrm{B}$ Breaking the $\mathrm{SO}(10)$ gauge symmetry}

In breaking the GUT symmetry to the symmetry of the standard model gauge group the fields that enter are $\mathcal{S}_{1_{126}}, \mathcal{S}_{1 \overline{126}}, \mathcal{S}_{1_{210}}, \mathcal{S}_{24_{210}}, \mathcal{S}_{75_{210}}$. Retaining these fields, eq. (2.1) 
takes the form

$$
\begin{aligned}
W_{\mathrm{GUT}}= & M^{126}\left(\frac{15}{2} \mathcal{S}_{1_{126}} \mathcal{S}_{1_{\overline{126}}}+\cdots\right)+M^{210}\left(\frac{3}{4} \mathcal{S}_{75_{210}}^{2}+\frac{5}{12} \mathcal{S}_{24_{210}}^{2}+\frac{3}{80} \mathcal{S}_{1_{210}}^{2}+\cdots\right) \\
+\eta(- & \left.\frac{3}{16} \mathcal{S}_{1_{210}} \mathcal{S}_{1_{126}} \mathcal{S}_{1 \overline{126}}+\cdots\right)+\lambda\left(\frac{1}{18} \mathcal{S}_{75_{210}}^{3}-\frac{1}{18} \mathcal{S}_{75_{210}}^{2} \mathcal{S}_{24_{210}}+\frac{25}{864} \mathcal{S}_{75_{210}} \mathcal{S}_{24_{210}}^{2}\right. \\
& \left.+\frac{3}{160} \mathcal{S}_{75_{210}}^{2} \mathcal{S}_{1_{210}}-\frac{35}{3888} \mathcal{S}_{24_{210}}^{3}-\frac{1}{192} \mathcal{S}_{24_{210}}^{2} \mathcal{S}_{1_{210}}-\frac{3}{3200} \mathcal{S}_{1_{210}}^{3}+\cdots\right) .
\end{aligned}
$$

As noted above, $\mathcal{S}_{1_{126}}, \mathcal{S}_{1 \overline{126}}, \mathcal{S}_{1_{210}}, \mathcal{S}_{24_{210}}, \mathcal{S}_{75_{210}}$ are the Standard Model singlets that acquire VEVs after normalization through eq. (A.5). Eq. (B.1) corrects the coefficient of the term $\mathcal{S}_{75_{210}}^{2} \mathcal{S}_{1_{210}}$ that appears in [28] by a factor of $3 / 4$ in agreement with the analysis of [77]. The spontaneous symmetry breaking equations including this factor are eqs. (B.2)(B.5). Vanishing of the $F$-terms leads to the immediate determination of $\mathcal{V}_{1_{210}}$ and a cubic equation in $\mathcal{V}_{24_{210}}$ through

$$
\begin{aligned}
\mathcal{V}_{1_{210}} & =2 \sqrt{15} \mathcal{M}^{126}, \\
\sqrt{15} \mathcal{V}_{24_{210}}^{3}+60 \mathcal{V}_{24_{210}}^{2}\left(3 \mathcal{M}^{126}-5 \mathcal{M}^{210}\right) & \\
+240 \sqrt{15} \mathcal{V}_{24_{210}}\left[3\left(\mathcal{M}^{126}\right)^{2}-5 \mathcal{M}^{126} \mathcal{M}^{210}+12\left(\mathcal{M}^{210}\right)^{2}\right] & \\
+14400\left(\mathcal{M}^{126}-2 \mathcal{M}^{210}\right)\left(\mathcal{M}^{126}+\mathcal{M}^{210}\right)^{2} & =0
\end{aligned}
$$

where the $\mathcal{V}$ 's are defined in eq. (A.6) and $\mathcal{M}^{126} \equiv \frac{M^{126}}{\eta}, \mathcal{M}^{210} \equiv \frac{M^{210}}{\lambda}$. [For an early work on the appearance of a cubic equation in spontaneous breaking of the GUT symmetry see [9]]. The remaining $\mathrm{SU}(3)_{C} \times \mathrm{SU}(2)_{L} \times \mathrm{U}(1)_{Y}$ singlet fields are functions of $\mathcal{V}_{24_{210}}$ and are determined by

$$
\begin{aligned}
\mathcal{V}_{75_{210}}= & \frac{\sqrt{15} \mathcal{V}_{24_{210}}^{2}+60 \mathcal{V}_{24210}\left(\mathcal{M}^{126}-2 \mathcal{M}^{210}\right)}{\sqrt{3} \mathcal{V}_{24_{210}}+12 \sqrt{5}\left(\mathcal{M}^{126}+\mathcal{M}^{210}\right)} \\
\mathcal{V}_{1_{126}} \cdot \mathcal{V}_{1_{126}}= & \frac{3}{32 \sqrt{5}}\left(\frac{\lambda}{\eta}\right) \frac{1}{\sqrt{3} \mathcal{V}_{24_{210}}+12 \sqrt{5}\left(\mathcal{M}^{126}+\mathcal{M}^{210}\right)} \\
& \times\left\{\sqrt{15} \mathcal{V}_{24_{210}}^{3}-120 \mathcal{V}_{24_{210}}^{2}\left(\mathcal{M}^{126}+9 \mathcal{M}^{210}\right)\right. \\
& -80 \sqrt{15} \mathcal{V}_{24_{210}}\left[21\left(\mathcal{M}^{126}\right)^{2}-17 \mathcal{M}^{126} \mathcal{M}^{210}-18\left(\mathcal{M}^{210}\right)^{2}\right] \\
& \left.-19200 \mathcal{M}^{126}\left(3 \mathcal{M}^{126}-2 \mathcal{M}^{210}\right)\left(\mathcal{M}^{126}+\mathcal{M}^{210}\right)\right\} .
\end{aligned}
$$

Finally, setting $D$-terms to zero yields

$$
\mathcal{V}_{1_{126}}=\mathcal{V}_{1_{126}}
$$




\section{Higgs doublet mass matrix}

A computation of the $7 \times 7$ doublet mass matrix was given in [28]. Here we record the matrix for completeness using the constraint of eq. (B.6). We have

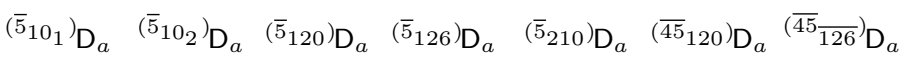

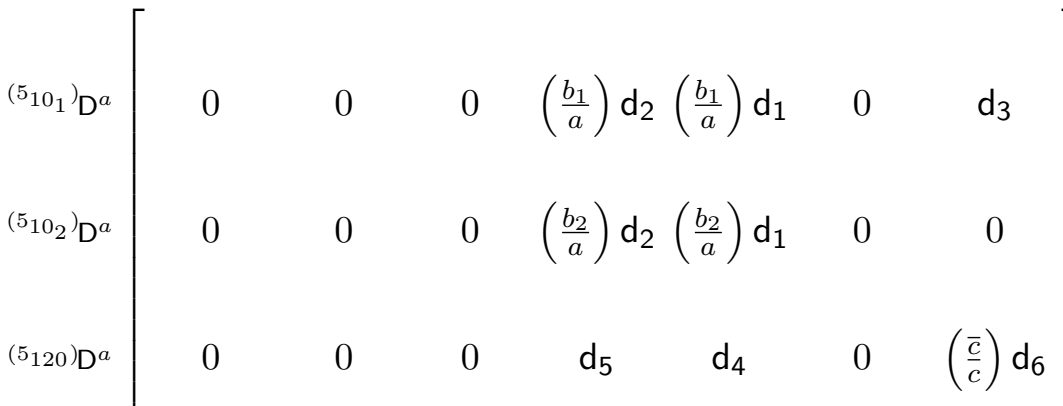

$$
\begin{aligned}
& M_{d}={ }^{(5 \overline{126})^{a}} \mathrm{D}^{a} \quad \begin{array}{llllllll}
\mathrm{d}_{2} & 0 & \left(\frac{\bar{c}}{c}\right) \mathrm{d}_{5} & \mathrm{~d}_{9} & \mathrm{~d}_{11} & \left(\frac{\bar{c}}{c}\right) \mathrm{d}_{7} & 0
\end{array} \\
& { }_{\left(45_{126}\right) \mathrm{D}^{a}}^{\left(5_{210}\right) \mathrm{D}^{a}}\left[\begin{array}{ccccccc}
\mathrm{d}_{1} & 0 & \left(\frac{\bar{c}}{c}\right) \mathrm{d}_{4} & \mathrm{~d}_{11} & \mathrm{~d}_{10} & 0 & 0 \\
0 & 0 & 0 & \mathrm{~d}_{7} & 0 & 0 & \left(\frac{\bar{c}}{c}\right) \mathrm{d}_{8} \\
\left(\frac{b_{1}}{a}\right) \mathrm{d}_{3}\left(\frac{b_{2}}{a}\right) \mathrm{d}_{3} & \mathrm{~d}_{6} & 0 & 0 & \mathrm{~d}_{8} & \mathrm{~d}_{12}
\end{array}\right] \\
& \mathrm{d}_{1} \equiv \frac{a}{2 \sqrt{5}} \mathcal{V}_{1_{126}} \text {, } \\
& \mathrm{d}_{2} \equiv-a\left[\frac{\sqrt{3}}{10} \mathcal{V}_{1_{210}}+\frac{\sqrt{3}}{20} \mathcal{V}_{24_{210}}\right] \text {, } \\
& \mathrm{d}_{3} \equiv a\left[-\frac{1}{4 \sqrt{6}} \mathcal{V}_{24_{210}}+\frac{1}{4 \sqrt{15}} \mathcal{V}_{75_{210}}\right] \text {, } \\
& \mathrm{d}_{4} \equiv-\frac{c}{\sqrt{30}} \mathcal{V}_{1_{126}} \text {, } \\
& \mathrm{d}_{5} \equiv c\left[-\frac{1}{10 \sqrt{2}} \mathcal{V}_{1_{210}}+\frac{3}{40 \sqrt{2}} \mathcal{V}_{24_{210}}\right] \text {, } \\
& \mathrm{d}_{6} \equiv-c\left[\frac{1}{48} \mathcal{V}_{24_{210}}+\frac{1}{12 \sqrt{10}} \mathcal{V}_{75_{210}}\right] \text {, } \\
& \mathrm{d}_{7} \equiv c\left[\frac{1}{48 \sqrt{3}} \mathcal{V}_{24_{210}}+\frac{1}{12 \sqrt{30}} \mathcal{V}_{75_{210}}\right] \text {, } \\
& \mathrm{d}_{8} \equiv-c\left[\frac{1}{20 \sqrt{6}} \mathcal{V}_{1_{210}}+\frac{1}{240 \sqrt{6}} \mathcal{V}_{24_{210}}+\frac{1}{12 \sqrt{15}} \mathcal{V}_{75_{210}}\right] \text {, } \\
& \mathrm{d}_{9} \equiv 2 M^{126}-\eta\left[\frac{2}{5 \sqrt{15}} \mathcal{V}_{1_{210}}+\frac{3}{20} \sqrt{\frac{3}{5}} \mathcal{V}_{24_{210}}\right] \text {, }
\end{aligned}
$$




$$
\begin{aligned}
\mathrm{d}_{10} & \equiv 2 M^{210}-\lambda\left[\frac{3}{10 \sqrt{2}} \mathcal{V}_{1_{210}}+\frac{1}{2} \sqrt{\frac{3}{5}} \mathcal{V}_{24_{210}}\right] \\
\mathrm{d}_{11} & \equiv \frac{1}{5} \eta \mathcal{V}_{1_{126}} \\
\mathrm{~d}_{12} & \equiv M^{126}+\eta\left[-\frac{1}{6 \sqrt{15}} \mathcal{V}_{24_{210}}+\frac{1}{15 \sqrt{6}} \mathcal{V}_{75_{210}}\right]
\end{aligned}
$$

\section{Details of Yukawa couplings from quartic interactions}

In this appendix we give details of the computations of the Yukawa couplings arising from the quartic interactions of $W_{4}^{(1)}, W_{4}^{(2)}$ and $W_{4}^{(3)}$ of the superpotential given in eqs. (4.2)(4.4). We discuss their contribution in that order.

1. We compute contriubtions from eq. (4.2) first.

$$
\begin{aligned}
& W_{4}^{(1)}=\frac{f^{(1)}}{5 ! M_{c}} b_{r}\left\langle\Psi_{(+)}^{*}\left|B \Gamma_{[\lambda} \Gamma_{\mu} \Gamma_{\nu} \Gamma_{\rho} \Gamma_{\sigma]}\right| \Psi_{(+)}\right\rangle\left[-{ }^{r} \Omega_{\lambda} \Phi_{\mu \nu \rho \sigma}+{ }^{r} \Omega_{\mu} \Phi_{\lambda \nu \rho \sigma}-{ }^{r} \Omega_{\nu} \Phi_{\lambda \mu \rho \sigma}\right. \\
& \left.+{ }^{r} \Omega_{\rho} \Phi_{\lambda \mu \nu \sigma}-{ }^{r} \Omega_{\sigma} \Phi_{\lambda \mu \nu \rho}\right] \\
& =-i \frac{f^{(1)}}{5 ! M_{c}} b_{r}\left[-15 \epsilon_{i j k l m} \mathrm{M}^{i j} \mathrm{M}^{n p} \mathrm{H}^{\left(10_{r}\right) k} \mathrm{H}_{n p}^{(210) l m}-\frac{15}{2} \epsilon_{i j k l m} \mathrm{M}^{i j} \mathrm{M}^{k n} \mathrm{H}^{\left(10_{r}\right) p} \mathrm{H}_{n p}^{(210) l m}\right. \\
& +\frac{5}{2} \epsilon_{i j k l m} \mathrm{M}^{i j} \mathrm{M}^{k l} \mathrm{H}^{\left(10_{r}\right) n} \mathrm{H}_{n}^{(210) m}-\frac{3}{4} \epsilon_{i j k l m} \mathrm{M}^{i j} \mathrm{M}^{k l} \mathrm{H}^{\left(10_{r}\right) m} \mathrm{H}^{(210)} \\
& -60 \mathrm{M}^{i j} \mathrm{M}_{k} \mathrm{H}_{l}^{\left(10_{r}\right)} \mathrm{H}_{i j}^{(210) k l}-20 \mathrm{M}^{i j} \mathrm{M}_{i} \mathrm{H}_{k}^{\left(10_{r}\right)} \mathrm{H}_{j}^{(210) k} \\
& \left.+80 \mathrm{M}^{i j} \mathrm{M}_{k} \mathrm{H}_{i}^{\left(10_{r}\right)} \mathrm{H}_{j}^{(210) k}+\cdots\right] \text {. }
\end{aligned}
$$

(a)

$$
\begin{aligned}
-15 \epsilon_{i j k l m} \mathrm{M}^{i j} \mathrm{M}^{n p} \mathrm{H}^{\left(10_{r}\right) k} \mathrm{H}_{n p}^{(210) l m}= & -60 \epsilon_{\gamma \sigma \beta a c} \mathrm{M}^{\gamma \sigma} \mathrm{M}^{b \alpha} \mathrm{H}^{\left(10_{r}\right) a} \mathrm{H}_{b \alpha}^{(210) c \beta} \\
& +15 \epsilon_{b a \rho \gamma \sigma} \mathrm{M}^{\alpha \beta} \mathrm{M}^{b \rho} \mathrm{H}^{\left(10_{r}\right) a} \mathrm{H}_{\alpha \beta}^{(210) \gamma \sigma}+\cdots
\end{aligned}
$$

i.

$$
-60 \epsilon_{\gamma \sigma \beta a c} \mathrm{M}^{\gamma \sigma} \mathrm{M}^{b \alpha} \mathrm{H}^{\left(10_{r}\right) a} \mathrm{H}_{b \alpha}^{(210) c \beta}=20 \mathcal{S}_{75_{210}} \epsilon_{a b} \mathbf{U}_{\alpha}^{c} \mathbf{Q}^{b \alpha} \mathbf{H}^{\left(10_{r}\right) a}
$$

ii.

$$
15 \epsilon_{b a \rho \gamma \sigma} \mathbf{M}^{\alpha \beta} \mathbf{M}^{b \rho} \mathbf{H}^{\left(10_{r}\right) a} \mathbf{H}_{\alpha \beta}^{(210) \gamma \sigma}=-10 \mathcal{S}_{75_{210}} \epsilon_{a b} \mathbf{U}_{\alpha}^{\mathrm{c}} \mathbf{Q}^{b \alpha} \mathbf{H}^{\left(10_{r}\right) a}
$$

(b)

$$
\begin{aligned}
-\frac{15}{2} \epsilon_{i j k l m} \mathrm{M}^{i j} \mathrm{M}^{k n} \mathrm{H}^{\left(10_{r}\right) p} \mathrm{H}_{n p}^{(210) l m}= & \frac{15}{2} \epsilon_{\alpha \beta \gamma c d} \mathrm{M}^{\alpha \beta} \mathrm{M}^{b \gamma} \mathrm{H}^{\left(10_{r}\right) a} \mathrm{H}_{b a}^{(210) c d} \\
& +15 \epsilon_{\gamma \sigma \beta c b} \mathrm{M}^{\gamma \sigma} \mathrm{M}^{c \alpha} \mathrm{H}^{\left(10_{r}\right) a} \mathrm{H}_{\alpha a}^{(210) \beta b} \\
& +30 \epsilon_{\sigma \gamma \beta c b} \mathrm{M}^{\gamma \alpha} \mathrm{M}^{c \sigma} \mathrm{H}^{\left(10_{r}\right) a} \mathrm{H}_{\alpha a}^{(210) \beta b}+\cdots
\end{aligned}
$$

i.

$$
\frac{15}{2} \epsilon_{\alpha \beta \gamma c d} \mathrm{M}^{\alpha \beta} \mathrm{M}^{b \gamma} \mathrm{H}^{\left(10_{r}\right) a} \mathrm{H}_{b a}^{(210) c d}=-15 \mathcal{S}_{75_{210}} \epsilon_{a b} \mathbf{U}_{\alpha}^{c} \mathbf{Q}^{b \alpha} \mathbf{H}^{\left(10_{r}\right) a}
$$


ii.

$$
15 \epsilon_{\gamma \sigma \beta c b} \mathrm{M}^{\gamma \sigma} \mathrm{M}^{c \alpha} \mathrm{H}^{\left(10_{r}\right) a} \mathrm{H}_{\alpha a}^{(210) \beta b}=\frac{5}{2} \mathcal{S}_{75_{210}} \epsilon_{a b} \mathbf{U}_{\alpha}^{c} \mathbf{Q}^{b \alpha} \mathrm{H}^{\left(10_{r}\right) a}
$$

iii.

$$
30 \epsilon_{\sigma \gamma \beta c b} \mathbf{M}^{\gamma \alpha} \mathbf{M}^{c \sigma} \mathbf{H}^{\left(10_{r}\right) a} \mathbf{H}_{\alpha a}^{(210) \beta b}=10 \mathcal{S}_{75_{210}} \epsilon_{a b} \mathbf{U}_{\alpha}^{c} \mathbf{Q}^{b \alpha} \mathbf{H}^{\left(10_{r}\right) a}
$$

(c)

$$
\frac{5}{2} \epsilon_{i j k l m} \mathrm{M}^{i j} \mathrm{M}^{k l} \mathrm{H}^{\left(10_{r}\right) n} \mathrm{H}_{n}^{(210) m}=-10 \mathcal{S}_{24_{210}} \epsilon_{a b} \mathbf{U}_{\alpha}^{\mathrm{c}} \mathbf{Q}^{b \alpha} \mathbf{H}^{\left(10_{r}\right) a}+\cdots
$$

(d)

$$
-\frac{3}{4} \epsilon_{i j k l m} \mathrm{M}^{i j} \mathrm{M}^{k l} \mathrm{H}^{\left(10_{r}\right) m} \mathbf{H}^{(210)}=-6 \mathcal{S}_{1_{210}} \epsilon_{a b} \mathbf{U}_{\alpha}^{c} \mathbf{Q}^{b \alpha} \mathbf{H}^{\left(10_{r}\right) a}+\cdots
$$

(e)

$$
-60 \mathrm{M}^{i j} \mathrm{M}_{k} \mathrm{H}_{l}^{\left(10_{r}\right)} \mathrm{H}_{i j}^{(210) k l}=60 \mathcal{S}_{75_{210}} \epsilon^{a b} \mathbf{E}^{\mathrm{c}} \mathbf{L}_{b} \mathrm{H}_{a}^{\left(10_{r}\right)}-20 \mathcal{S}_{75_{210}} \mathbf{Q}^{a \alpha} \mathbf{D}_{\alpha}^{\mathrm{c}} \mathrm{H}_{a}^{\left(10_{r}\right)}+\cdots
$$

(f)

$$
-20 \mathrm{M}^{i j} \mathrm{M}_{i} \mathrm{H}_{k}^{\left(10_{r}\right)} \mathrm{H}_{j}^{(210) k}=-10 \mathcal{S}_{24_{210}} \epsilon^{a b} \mathbf{E}^{\mathrm{c}} \mathbf{L}_{b} \mathrm{H}_{a}^{\left(10_{r}\right)}-10 \mathcal{S}_{24_{210}} \mathbf{Q}^{a \alpha} \mathbf{D}_{\alpha}^{\mathrm{c}} \mathrm{H}_{a}^{\left(10_{r}\right)}+\cdots
$$

(g)

$$
80 \mathrm{M}^{i j} \mathrm{M}_{k} \mathrm{H}_{i}^{\left(10_{r}\right)} \mathrm{H}_{j}^{(210) k}=-40 \mathcal{S}_{24_{210}} \epsilon^{a b} \mathbf{E}^{\mathrm{c}} \mathbf{L}_{b} \mathrm{H}_{a}^{\left(10_{r}\right)}+\frac{80}{3} \mathcal{S}_{24_{210}} \mathbf{Q}^{a \alpha} \mathbf{D}_{\alpha}^{\mathrm{c}} \mathrm{H}_{a}^{\left(10_{r}\right)}+\cdots
$$

Thus,

$$
\begin{aligned}
W_{4}^{(1)}=-i \frac{f^{(1)}}{5 ! M_{c}} b_{r}[ & \left(\frac{15}{2} \mathcal{S}_{75_{210}}-10 \mathcal{S}_{24_{210}}-6 \mathcal{S}_{1_{210}}\right) \epsilon_{a b} \mathbf{U}_{\alpha}^{\mathrm{c}} \mathbf{Q}^{b \alpha} \mathbf{H}^{\left(10_{r}\right) a} \\
& +\left(-20 \mathcal{S}_{75_{210}}+\frac{50}{3} \mathcal{S}_{24_{210}}\right) \mathbf{D}_{\alpha}^{\mathrm{c}} \mathbf{Q}^{a \alpha} \mathbf{H}_{a}^{\left(10_{r}\right)} \\
& \left.+\left(60 \mathcal{S}_{75_{210}}-50 \mathcal{S}_{24_{210}}\right) \epsilon^{a b} \mathbf{E}^{\mathrm{c}} \mathbf{L}_{b} \mathrm{H}_{a}^{\left(10_{r}\right)}+\cdots\right]
\end{aligned}
$$

Using $\epsilon_{54}=-1=\epsilon^{54}$ and $\epsilon_{45}=+1=\epsilon^{45}$, we get

$$
\begin{aligned}
\epsilon_{a b} \mathbf{U}_{\alpha}^{c} \mathbf{Q}^{b \alpha} \mathbf{H}^{\left(10_{r}\right) a} & =-\mathbf{U}_{\alpha}^{c} \mathbf{U}^{\alpha} \mathbf{H}^{\left(10_{r}\right) 5}+\cdots, \\
\mathbf{D}_{\alpha}^{c} \mathbf{Q}^{a \alpha} \mathbf{H}_{a}^{\left(10_{r}\right)} & =\mathbf{D}_{\alpha}^{\mathrm{c}} \mathbf{D}^{\alpha} \mathbf{H}_{5}^{\left(10_{r}\right)}+\cdots \\
\epsilon^{a b} \mathbf{E}^{\mathrm{c}} \mathbf{L}_{b} \mathbf{H}_{a}^{\left(10_{r}\right)} & =-\mathbf{E}^{\mathrm{c}} \mathbf{E} \mathbf{H}_{5}^{\left(10_{r}\right)}+\cdots
\end{aligned}
$$

Further, on using eqs. (2.5), (A.2) and (A.3) gives

$$
\begin{aligned}
& \mathbf{H}^{\left(10_{1}\right) 5} \equiv{ }^{\left(5_{10}\right)_{1}} \mathcal{D}^{5}=\sqrt{2}{ }^{\left(5_{10_{1}}\right)} \mathbf{D}^{5}=\sqrt{2} U_{d_{11}}\left\langle H_{u}\right\rangle+\cdots, \\
& \mathrm{H}^{\left(10_{2}\right) 5} \equiv{ }^{\left(5_{10_{2}}\right)} \mathcal{D}^{5}=\sqrt{2}{ }^{\left(5_{10_{2}}\right)} \mathrm{D}^{5}=\sqrt{2} U_{d_{21}}\left\langle H_{u}\right\rangle+\cdots,
\end{aligned}
$$

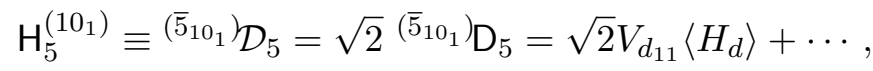

$$
\begin{aligned}
& \mathrm{H}_{5}^{\left(10_{2}\right)} \equiv{ }^{\left(\overline{5}_{10_{2}}\right)} \mathcal{D}_{5}=\sqrt{2}{ }^{\left(\overline{5}_{10_{2}}\right)} \mathrm{D}_{5}=\sqrt{2} V_{d_{21}}\left\langle H_{d}\right\rangle+\cdots,
\end{aligned}
$$

where, $H_{d} \equiv \mathbf{H}_{\mathbf{d} 5}$ and $H_{u} \equiv \mathbf{H}_{\mathbf{u}}{ }^{5}$. 
And finally, using (see eqs. (A.5) and (A.6)),

$$
\begin{aligned}
\left\langle\mathcal{S}_{1_{210}}\right\rangle & =4 \sqrt{\frac{5}{3}} \mathcal{V}_{1_{210}}, \\
\left\langle\mathcal{S}_{24_{210}}\right\rangle & =2 \sqrt{\frac{3}{5}} \mathcal{V}_{24_{210}}, \\
\left\langle\mathcal{S}_{75_{210}}\right\rangle & =\frac{1}{\sqrt{3}} \mathcal{V}_{24_{210}},
\end{aligned}
$$

gives eqs. (4.5)-(4.7).

2. Next we compute contribution to the Yukawas of the third generation from eq. (4.3).

$$
\begin{aligned}
W_{4}^{(2)}=\frac{f^{(2)}}{5 ! M_{c}}\left\langle\Psi_{(+)}^{*}\left|B \Gamma_{[\lambda} \Gamma_{\mu} \Gamma_{\nu} \Gamma_{\rho} \Gamma_{\sigma]}\right| \Psi_{(+)}\right\rangle & {\left[-\Sigma_{\lambda \alpha \beta} \Phi_{\gamma \rho \sigma \lambda}+\Sigma_{\lambda \alpha \gamma} \Phi_{\beta \rho \sigma \lambda}-\Sigma_{\lambda \alpha \rho} \Phi_{\beta \gamma \sigma \lambda}\right.} \\
& +\Sigma_{\lambda \alpha \sigma} \Phi_{\beta \gamma \rho \lambda}+\Sigma_{\lambda \gamma \beta} \Phi_{\alpha \rho \sigma \lambda}-\Sigma_{\lambda \rho \beta} \Phi_{\alpha \gamma \sigma \lambda} \\
& +\Sigma_{\lambda \sigma \beta} \Phi_{\alpha \gamma \rho \lambda}+\Sigma_{\lambda \gamma \rho} \Phi_{\beta \alpha \sigma \lambda}-\Sigma_{\lambda \gamma \sigma} \Phi_{\beta \alpha \rho \lambda} \\
& \left.+\Sigma_{\lambda \rho \sigma} \Phi_{\beta \alpha \gamma \lambda}\right] \\
=-i \frac{f^{(2)}}{5 ! M_{c}}[ & -\frac{15}{2} \epsilon_{i j k l m} \mathrm{M}^{i j} \mathrm{M}^{n o} \mathrm{H}_{x}^{(120) k l} \mathrm{H}_{n o}^{(210) m x}+15 \epsilon_{i j k l m} \mathrm{M}^{i j} \mathrm{M}^{n o} \mathrm{H}_{n}^{(120) x k} \mathrm{H}_{x o}^{(210) l m} \\
& +5 \epsilon_{i j k l m} \mathrm{M}^{i j} \mathrm{M}^{n o} \mathrm{H}_{n}^{(120) k l} \mathrm{H}_{o}^{(210) m}+\frac{15}{2} \epsilon_{i j k l m} \mathrm{M}^{i j} \mathrm{M}^{k n} \mathrm{H}_{n}^{(120) x y} \mathrm{H}_{x y}^{(210) l m} \\
& -\frac{5}{2} \epsilon_{i j k l m} \mathrm{M}^{i j} \mathrm{M}^{k n} \mathrm{H}_{x}^{(120) l m} \mathrm{H}_{n}^{(210) x}+5 \epsilon_{i j k l m} \mathrm{M}^{i j} \mathrm{M}^{k n} \mathrm{H}_{n}^{(120) x l} \mathrm{H}_{x}^{(210) m} \\
& +\frac{15}{8} \epsilon_{i j k l m} \mathrm{M}^{i j} \mathrm{M}^{k l} \mathrm{H}_{z}^{(120) x y} \mathrm{H}_{x y}^{(210) m z}-\frac{5}{4} \epsilon_{i j k l m} \mathrm{M}^{i j} \mathrm{M}^{k l} \mathrm{H}_{y}^{(120) m x} \mathrm{H}_{x}^{(210) y} \\
& +\frac{3}{16} \epsilon_{i j k l m} \mathrm{M}^{i j} \mathrm{M}^{k l} \mathrm{H}^{(120) m} \mathrm{H}^{(210)}+\frac{15}{16} \epsilon_{i j k l m} \mathrm{M}^{i j} \mathrm{M}^{k l} \mathrm{H}^{(120) x} \mathrm{H}_{x}^{(210) m} \\
& -15 \mathrm{M}^{i j} \mathrm{M}_{j} \mathrm{H}_{y z}^{(120) x} \mathrm{H}_{i x}^{(210) y z}+10 \mathrm{M}^{i j} \mathrm{M}_{j} \mathrm{H}_{i y}^{(120) x} \mathrm{H}_{x}^{(210) y} \\
& -\frac{5}{2} \mathrm{M}^{i j} \mathrm{M}_{j} \mathrm{H}_{x}^{(120)} \mathrm{H}_{i}^{(210) x}-20 \mathrm{M}^{i j} \mathrm{M}_{k} \mathrm{H}_{j x}^{(120) k} \mathrm{H}_{i}^{(210) x} \\
& -30 \mathrm{M}^{i j} \mathrm{M}_{k} \mathrm{H}_{i j}^{(120) x} \mathrm{H}_{x}^{(210) k}+30 \mathrm{M}^{i j} \mathrm{M}_{k} \mathrm{H}_{x y}^{(120) k} \mathrm{H}_{i j}^{(210) x y} \\
& +3 \mathrm{M}^{i j} \mathrm{M}_{k} \mathrm{H}_{i j}^{(120) k} \mathrm{H}^{(210)}-10 \mathrm{M}^{i j} \mathrm{M}_{k} \mathrm{H}_{i}^{(120)} \mathrm{H}_{j}^{(210) k} \\
& \left.-15 \mathrm{M}^{i j} \mathrm{M}_{k} \mathrm{H}_{x}^{(120)} \mathrm{H}_{i j}^{(210) k x}+\cdots\right]
\end{aligned}
$$

(a)

$$
\begin{aligned}
-\frac{15}{2} \epsilon_{i j k l m} \mathrm{M}^{i j} \mathrm{M}^{n o} \mathrm{H}_{x}^{(120) k l} \mathrm{H}_{n o}^{(210) m x}=-15 & {\left[-\epsilon_{\beta \gamma \alpha a b} \mathrm{M}^{\beta \gamma} \mathrm{M}^{d \rho} \mathrm{H}_{c}^{(120) a b} \mathrm{H}_{\rho d}^{(210) \alpha c}\right.} \\
& +2 \epsilon_{\gamma \rho \alpha a b} \mathrm{M}^{\gamma \rho} \mathrm{M}^{d \sigma} \mathrm{H}_{\beta}^{(120) \alpha a} \mathrm{H}_{d \sigma}^{(210) b \beta} \\
& \left.+2 \epsilon_{\gamma \lambda \alpha b a} \mathrm{M}^{\rho \sigma} \mathrm{M}^{b \lambda} \mathrm{H}_{\beta}^{(120) \alpha a} \mathrm{H}_{\rho \sigma}^{(210) \gamma \beta}+\cdots\right]
\end{aligned}
$$

i.

$$
-\epsilon_{\beta \gamma \alpha a b} \mathrm{M}^{\beta \gamma} \mathrm{M}^{d \rho} \mathrm{H}_{c}^{(120) a b} \mathrm{H}_{\rho d}^{(210) \alpha c}=-\frac{2}{3} \mathcal{S}_{75_{210}} \epsilon_{a b} \mathbf{U}_{\alpha}^{c} \mathbf{Q}^{a \alpha}\left(45_{120}\right) \mathcal{D}^{b}
$$


ii.

$$
2 \epsilon_{\gamma \rho \alpha a b} \mathbf{M}^{\gamma \rho} \mathbf{M}^{d \sigma} \mathbf{H}_{\beta}^{(120) \alpha a} \mathbf{H}_{d \sigma}^{(210) b \beta}=\frac{2}{9} \mathcal{S}_{75_{210}} \epsilon_{a b} \mathbf{U}_{\alpha}^{c} \mathbf{Q}^{a \alpha}{ }^{\left(45_{120}\right)} \mathcal{D}^{b}
$$

iii.

$$
2 \epsilon_{\gamma \lambda \alpha b a} \mathrm{M}^{\rho \sigma} \mathbf{M}^{b \lambda} \mathbf{H}_{\beta}^{(120) \alpha a} \mathbf{H}_{\rho \sigma}^{(210) \gamma \beta}=-\frac{4}{9} \mathcal{S}_{75_{210}} \epsilon_{a b} \mathbf{U}_{\alpha}^{c} \mathbf{Q}^{a \alpha}\left(45_{120}\right) \mathcal{D}^{b}
$$

(b)

$$
\begin{aligned}
15 \epsilon_{i j k l m} \mathrm{M}^{i j} \mathrm{M}^{n o} \mathrm{H}_{n}^{(120) x k} \mathrm{H}_{x o}^{(210) l m}=15[ & 2 \epsilon_{\alpha \beta \rho c d} \mathrm{M}^{\alpha \beta} \mathrm{M}^{a \gamma} \mathrm{H}_{a}^{(120) b c} \mathrm{H}_{\gamma b}^{(210) \rho d} \\
& +\epsilon_{\alpha \beta \rho c d} \mathrm{M}^{\alpha \beta} \mathrm{M}^{b \gamma} \mathrm{H}_{\gamma}^{(120) \rho a} \mathrm{H}_{a b}^{(210) c d} \\
& +2 \epsilon_{\alpha \beta \sigma a c} \mathrm{M}^{\alpha \beta} \mathrm{M}^{b \gamma} \mathrm{H}_{\gamma}^{(120) \rho a} \mathrm{H}_{\rho b}^{(210) \sigma c} \\
& -4 \epsilon_{\alpha \rho \sigma a c} \mathrm{M}^{\beta \gamma} \mathrm{M}^{a \alpha} \mathrm{H}_{\beta}^{(120) \rho b} \mathrm{H}_{\gamma b}^{(210) \sigma c} \\
& \left.-2 \epsilon_{\alpha \sigma \lambda a b} \mathrm{M}^{\beta \gamma} \mathrm{M}^{a \alpha} \mathrm{H}_{\beta}^{(120) \rho b} \mathrm{H}_{\rho \gamma}^{(210) \sigma \lambda}+\cdots\right]
\end{aligned}
$$

i.

$$
2 \epsilon_{\alpha \beta \rho c d} \mathbf{M}^{\alpha \beta} \mathbf{M}^{a \gamma} \mathbf{H}_{a}^{(120) b c} \mathbf{H}_{\gamma b}^{(210) \rho d}=-\frac{4}{3} \mathcal{S}_{75_{210}} \epsilon_{a b} \mathbf{U}_{\alpha}^{c} \mathbf{Q}^{a \alpha}\left(45_{120}\right) \mathcal{D}^{b}
$$

ii.

$$
\epsilon_{\alpha \beta \rho c d} \mathrm{M}^{\alpha \beta} \mathrm{M}^{b \gamma} \mathbf{H}_{\gamma}^{(120) \rho a} \mathrm{H}_{a b}^{(210) c d}=-\frac{2}{3} \mathcal{S}_{75_{210}} \epsilon_{a b} \mathbf{U}_{\alpha}^{\mathrm{c}} \mathbf{Q}^{a \alpha\left(45_{120}\right)} \mathcal{D}^{b}
$$

iii.

$$
2 \epsilon_{\alpha \beta \sigma a c} \mathbf{M}^{\alpha \beta} \mathbf{M}^{b \gamma} \mathbf{H}_{\gamma}^{(120) \rho a} \mathbf{H}_{\rho b}^{(210) \sigma c}=\frac{2}{9} \mathcal{S}_{75_{210}} \epsilon_{a b} \mathbf{U}_{\alpha}^{c} \mathbf{Q}^{a \alpha\left(45_{120}\right)} \mathcal{D}^{b}
$$

iv.

$$
-4 \epsilon_{\alpha \rho \sigma a c} \mathrm{M}^{\beta \gamma} \mathrm{M}^{a \alpha} \mathbf{H}_{\beta}^{(120) \rho b} \mathbf{H}_{\gamma b}^{(210) \sigma c}=\frac{4}{9} \mathcal{S}_{75_{210}} \epsilon_{a b} \mathbf{U}_{\alpha}^{c} \mathbf{Q}^{a \alpha}\left(45_{120}\right) \mathcal{D}^{b}
$$

v.

$$
-2 \epsilon_{\alpha \sigma \lambda a b} \mathrm{M}^{\beta \gamma} \mathrm{M}^{a \alpha} \mathrm{H}_{\beta}^{(120) \rho b} \mathrm{H}_{\rho \gamma}^{(210) \sigma \lambda}=-\frac{4}{9} \mathcal{S}_{75_{210}} \epsilon_{a b} \mathbf{U}_{\alpha}^{\mathrm{c}} \mathbf{Q}^{a \alpha}\left(45_{120}\right) \mathcal{D}^{b}
$$

(c)

$$
\begin{aligned}
5 \epsilon_{i j k l m} \mathrm{M}^{i j} \mathrm{M}^{n o} \mathrm{H}_{n}^{(120) k l} \mathrm{H}_{o}^{(210) m}=5[ & -2 \epsilon_{\alpha \beta \rho c a} \mathrm{M}^{\alpha \beta} \mathrm{M}^{b \gamma} \mathrm{H}_{\gamma}^{(120) \rho c} \mathrm{H}_{b}^{(210) a} \\
& +\epsilon_{\alpha \beta \gamma b c} \mathrm{M}^{\alpha \beta} \mathrm{M}^{a \rho} \mathrm{H}_{a}^{(120) b c} \mathrm{H}_{\rho}^{(210) \gamma} \\
& \left.+4 \epsilon_{\alpha \sigma \beta a b} \mathrm{M}^{\rho \gamma} \mathrm{M}^{a \alpha} \mathrm{H}_{\rho}^{(120) \sigma b} \mathrm{H}_{\gamma}^{(210) \beta}+\cdots\right]
\end{aligned}
$$

i.

$$
-2 \epsilon_{\alpha \rho c a} \mathrm{M}^{\alpha \beta} \mathrm{M}^{b \gamma} \mathbf{H}_{\gamma}^{(120) \rho c} \mathrm{H}_{b}^{(210) a}=-\frac{2}{3} \mathcal{S}_{24_{210}} \epsilon_{a b} \mathbf{U}_{\alpha}^{\mathrm{c}} \mathbf{Q}^{a \alpha}\left(45_{120}\right) \mathcal{D}^{b}
$$

ii.

$$
\epsilon_{\alpha \beta \gamma b c} \mathrm{M}^{\alpha \beta} \mathrm{M}^{a \rho} \mathrm{H}_{a}^{(120) b c} \mathrm{H}_{\rho}^{(210) \gamma}=-\frac{4}{3} \mathcal{S}_{24_{210}} \epsilon_{a b} \mathbf{U}_{\alpha}^{\mathrm{c}} \mathbf{Q}^{a \alpha\left(45_{120}\right)} \mathcal{D}^{b}
$$

iii.

$$
4 \epsilon_{\alpha \sigma \beta a b} \mathrm{M}^{\rho \gamma} \mathrm{M}^{a \alpha} \mathrm{H}_{\rho}^{(120) \sigma b} \mathrm{H}_{\gamma}^{(210) \beta}=\frac{8}{9} \mathcal{S}_{24_{210}} \epsilon_{a b} \mathbf{U}_{\alpha}^{c} \mathbf{Q}^{a \alpha}\left(45_{120}\right) \mathcal{D}^{b}
$$


(d)

$$
\begin{aligned}
\frac{15}{2} \epsilon_{i j k l m} \mathrm{M}^{i j} \mathrm{M}^{k n} \mathrm{H}_{n}^{(120) x y} \mathrm{H}_{x y}^{(210) l m}=\frac{15}{2}[ & -4 \epsilon_{\alpha \beta \rho a b} \mathrm{M}^{\alpha \beta} \mathrm{M}^{a \gamma} \mathrm{H}_{\gamma}^{(120) \sigma c} \mathrm{H}_{c \sigma}^{(210) b \rho} \\
& -\epsilon_{\alpha \beta \gamma a b} \mathrm{M}^{\alpha \beta} \mathrm{M}^{c \gamma} \mathrm{H}_{c}^{(120) d e} \mathrm{H}_{d e}^{(210) a b} \\
& \left.-8 \epsilon_{\alpha \beta \rho a b} \mathrm{M}^{\beta \gamma} \mathrm{M}^{a \alpha} \mathrm{H}_{\gamma}^{(120) \sigma c} \mathrm{H}_{\sigma c}^{(210) \rho b}+\cdots\right]
\end{aligned}
$$

i.

$$
-4 \epsilon_{\alpha \beta \rho a b} \mathrm{M}^{\alpha \beta} \mathrm{M}^{a \gamma} \mathrm{H}_{\gamma}^{(120) \sigma c} \mathrm{H}_{c \sigma}^{(210) b \rho}=\frac{4}{9} \mathcal{S}_{75_{210}} \epsilon_{a b} \mathbf{U}_{\alpha}^{c} \mathbf{Q}^{a \alpha}{ }^{\left(45_{120}\right)} \mathcal{D}^{b}
$$

ii.

$$
-\epsilon_{\alpha \beta \gamma a b} \mathrm{M}^{\alpha \beta} \mathrm{M}^{c \gamma} \mathbf{H}_{c}^{(120) d e} \mathbf{H}_{d e}^{(210) a b}=4 \mathcal{S}_{75_{210}} \epsilon_{a b} \mathbf{U}_{\alpha}^{c} \mathbf{Q}^{a \alpha}\left(45_{120}\right) \mathcal{D}^{b}
$$

iii.

$$
-8 \epsilon_{\alpha \beta \rho a b} \mathrm{M}^{\beta \gamma} \mathrm{M}^{a \alpha} \mathrm{H}_{\gamma}^{(120) \sigma c} \mathrm{H}_{\sigma c}^{(210) \rho b}=\frac{8}{9} \mathcal{S}_{75_{210}} \epsilon_{a b} \mathbf{U}_{\alpha}^{c} \mathbf{Q}^{a \alpha}{ }^{\left(45_{120}\right)} \mathcal{D}^{b}
$$

(e)

$$
\begin{aligned}
-\frac{5}{2} \epsilon_{i j k l m} \mathrm{M}^{i j} \mathrm{M}^{k n} \mathrm{H}_{x}^{(120) l m} \mathrm{H}_{n}^{(210) x}=-\frac{5}{2}[ & -2 \epsilon_{\alpha \beta \sigma a b} \mathrm{M}^{\alpha \beta} \mathrm{M}^{a \gamma} \mathrm{H}_{\rho}^{(120) \sigma b} \mathrm{H}_{\gamma}^{(210) \rho} \\
& -\epsilon_{\alpha \beta \gamma c d} \mathrm{M}^{\alpha \beta} \mathrm{M}^{a \gamma} \mathrm{H}_{b}^{(120) c d} \mathrm{H}_{a}^{(210) b} \\
& \left.-4 \epsilon_{\alpha \beta \sigma a b} \mathrm{M}^{\beta \gamma} \mathrm{M}^{a \alpha} \mathrm{H}_{\rho}^{(120) \sigma b} \mathrm{H}_{\gamma}^{(210) \rho}+\cdots\right]
\end{aligned}
$$

i.

$$
-2 \epsilon_{\alpha \beta \sigma a b} \mathbf{M}^{\alpha \beta} \mathbf{M}^{a \gamma} \mathbf{H}_{\rho}^{(120) \sigma b} \mathbf{H}_{\gamma}^{(210) \rho}=-\frac{4}{9} \mathcal{S}_{24_{210}} \epsilon_{a b} \mathbf{U}_{\alpha}^{c} \mathbf{Q}^{a \alpha}{ }^{\left(45_{120}\right)} \mathcal{D}^{b}
$$

ii.

$$
-\epsilon_{\alpha \beta \gamma c d} \mathrm{M}^{\alpha \beta} \mathrm{M}^{a \gamma} \mathbf{H}_{b}^{(120) c d} \mathbf{H}_{a}^{(210) b}=-2 \mathcal{S}_{24_{210}} \epsilon_{a b} \mathbf{U}_{\alpha}^{\mathrm{c}} \mathbf{Q}^{a \alpha}{ }^{\left(45_{120}\right)} \mathcal{D}^{b}
$$

iii.

$$
-4 \epsilon_{\alpha \beta \sigma a b} \mathrm{M}^{\beta \gamma} \mathbf{M}^{a \alpha} \mathbf{H}_{\rho}^{(120) \sigma b} \mathbf{H}_{\gamma}^{(210) \rho}=-\frac{8}{9} \mathcal{S}_{24_{210}} \epsilon_{a b} \mathbf{U}_{\alpha}^{c} \mathbf{Q}^{a \alpha}{ }^{\left(45_{120}\right)} \mathcal{D}^{b}
$$

(f)

$$
\begin{aligned}
5 \epsilon_{i j k l m} \mathrm{M}^{i j} \mathrm{M}^{k n} \mathrm{H}_{n}^{(120) x l} \mathrm{H}_{x}^{(210) m}=5[ & \epsilon_{\alpha \beta \lambda a c} \mathrm{M}^{\alpha \beta} \mathrm{M}^{a \gamma} \mathrm{H}_{\gamma}^{(120) \lambda b} \mathrm{H}_{b}^{(210) c} \\
& +\epsilon_{\alpha \beta \lambda a b} \mathrm{M}^{\alpha \beta} \mathrm{M}^{a \gamma} \mathrm{H}_{\gamma}^{(120) \rho b} \mathrm{H}_{\rho}^{(210) \lambda} \\
& -\epsilon_{\alpha \beta \gamma b c} \mathrm{M}^{\alpha \beta} \mathrm{M}^{a \gamma} \mathrm{H}_{a}^{(120) d b} \mathrm{H}_{d}^{(210) c} \\
& +2 \epsilon_{\alpha \beta \rho a c} \mathrm{M}^{\beta \gamma} \mathrm{M}^{a \alpha} \mathrm{H}_{\gamma}^{(120) \rho b} \mathrm{H}_{b}^{(210) c} \\
& \left.+2 \epsilon_{\alpha \beta \lambda a b} \mathrm{M}^{\beta \gamma} \mathrm{M}^{a \alpha} \mathrm{H}_{\gamma}^{(120) \rho b} \mathrm{H}_{\rho}^{(210) \lambda}+\cdots\right]
\end{aligned}
$$

i.

ii.

$$
\epsilon_{\alpha \beta \lambda a c} \mathrm{M}^{\alpha \beta} \mathrm{M}^{a \gamma} \mathrm{H}_{\gamma}^{(120) \lambda b} \mathrm{H}_{b}^{(210) c}=-\frac{1}{3} \mathcal{S}_{24_{210}} \epsilon_{a b} \mathbf{U}_{\alpha}^{c} \mathbf{Q}^{a \alpha}\left(45_{120}\right) \mathcal{D}^{b}
$$

iii.

$$
\epsilon_{\alpha \beta \lambda a b} \mathrm{M}^{\alpha \beta} \mathrm{M}^{a \gamma} \mathrm{H}_{\gamma}^{(120) \rho b} \mathrm{H}_{\rho}^{(210) \lambda}=\frac{2}{9} \mathcal{S}_{24_{210}} \epsilon_{a b} \mathbf{U}_{\alpha}^{c} \mathbf{Q}^{a \alpha}\left(45_{120}\right) \mathcal{D}^{b}
$$

$$
-\epsilon_{\alpha \beta \gamma b c} \mathrm{M}^{\alpha \beta} \mathrm{M}^{a \gamma} \mathrm{H}_{a}^{(120) d b} \mathrm{H}_{d}^{(210) c}=2 \mathcal{S}_{24_{210}} \epsilon_{a b} \mathbf{U}_{\alpha}^{\mathrm{c}} \mathbf{Q}^{a \alpha\left(45_{120}\right)} \mathcal{D}^{b}
$$


iv.

$$
2 \epsilon_{\alpha \beta \rho a c} \mathrm{M}^{\beta \gamma} \mathrm{M}^{a \alpha} \mathrm{H}_{\gamma}^{(120) \rho b} \mathrm{H}_{b}^{(210) c}=-\frac{2}{3} \mathcal{S}_{24_{210}} \epsilon_{a b} \mathbf{U}_{\alpha}^{c} \mathbf{Q}^{a \alpha}{ }^{\left(45_{120}\right)} \mathcal{D}^{b}
$$

v.

$$
2 \epsilon_{\alpha \beta \lambda a b} \mathrm{M}^{\beta \gamma} \mathrm{M}^{a \alpha} \mathrm{H}_{\gamma}^{(120) \rho b} \mathrm{H}_{\rho}^{(210) \lambda}=\frac{4}{9} \mathcal{S}_{24_{210}} \epsilon_{a b} \mathbf{U}_{\alpha}^{c} \mathbf{Q}^{a \alpha\left(45_{120}\right)} \mathcal{D}^{b}
$$

(g)

$$
\begin{aligned}
\frac{15}{8} \epsilon_{i j k l m} \mathrm{M}^{i j} \mathrm{M}^{k l} \mathrm{H}_{z}^{(120) x y} \mathrm{H}_{x y}^{(210) m z}=-15 & {\left[\frac{1}{2} \epsilon_{\alpha \beta \gamma a b} \mathrm{M}^{\beta \gamma} \mathrm{M}^{a \alpha} \mathrm{H}_{c}^{(120) d e} \mathrm{H}_{d e}^{(210) b c}\right.} \\
& \left.-\epsilon_{\alpha \beta \gamma a b} \mathrm{M}^{\beta \gamma} \mathrm{M}^{a \alpha} \mathrm{H}_{\rho}^{(120) \sigma c} \mathrm{H}_{c \sigma}^{(210) b \rho}+\cdots\right]
\end{aligned}
$$

i.

$$
\frac{1}{2} \epsilon_{\alpha \beta \gamma a b} \mathrm{M}^{\beta \gamma} \mathbf{M}^{a \alpha} \mathbf{H}_{c}^{(120) d e} \mathbf{H}_{d e}^{(210) b c}=\mathcal{S}_{75_{210}} \epsilon_{a b} \mathbf{U}_{\alpha}^{c} \mathbf{Q}^{a \alpha}\left(45_{120}\right) \mathcal{D}^{b}
$$

ii.

$$
-\epsilon_{\alpha \beta \gamma a b} \mathrm{M}^{\beta \gamma} \mathrm{M}^{a \alpha} \mathbf{H}_{\rho}^{(120) \sigma c} \mathrm{H}_{c \sigma}^{(210) b \rho}=\frac{1}{3} \mathcal{S}_{75_{210}} \epsilon_{a b} \mathbf{U}_{\alpha}^{c} \mathbf{Q}^{a \alpha}\left(45_{120}\right) \mathcal{D}^{b}
$$

(h)

$$
\begin{aligned}
-\frac{5}{4} \epsilon_{i j k l m} \mathrm{M}^{i j} \mathrm{M}^{k l} \mathrm{H}_{y}^{(120) m x} \mathrm{H}_{x}^{(210) y}=5[ & \epsilon_{\alpha \beta \gamma a b} \mathrm{M}^{\beta \gamma} \mathrm{M}^{a \alpha} \mathrm{H}_{d}^{(120) b c} \mathrm{H}_{c}^{(210) d} \\
& \left.-\epsilon_{\alpha \beta \gamma a b} \mathrm{M}^{\beta \gamma} \mathrm{M}^{a \alpha} \mathrm{H}_{\sigma}^{(120) \rho b} \mathrm{H}_{\rho}^{(210) \sigma}+\cdots\right]
\end{aligned}
$$

i.

$$
\epsilon_{\alpha \beta \gamma a b} \mathrm{M}^{\beta \gamma} \mathbf{M}^{a \alpha} \mathbf{H}_{d}^{(120) b c} \mathbf{H}_{c}^{(210) d}=-\mathcal{S}_{24_{210}} \epsilon_{a b} \mathbf{U}_{\alpha}^{c} \mathbf{Q}^{a \alpha}{ }^{\left(45_{120}\right)} \mathcal{D}^{b}
$$

ii.

$$
-\epsilon_{\alpha \beta \gamma a b} \mathrm{M}^{\beta \gamma} \mathrm{M}^{a \alpha} \mathbf{H}_{\sigma}^{(120) \rho b} \mathbf{H}_{\rho}^{(210) \sigma}=-\frac{2}{3} \mathcal{S}_{24_{210}} \epsilon_{a b} \mathbf{U}_{\alpha}^{\mathrm{c}} \mathbf{Q}^{a \alpha}\left(45_{120}\right) \mathcal{D}^{b}
$$

(i)

$$
\frac{3}{16} \epsilon_{i j k l m} \mathrm{M}^{i j} \mathrm{M}^{k l} \mathrm{H}^{(120) m} \mathrm{H}^{(210)}=-\frac{3}{2} \mathcal{S}_{1_{210}} \epsilon_{a b} \mathbf{U}_{\alpha}^{\mathrm{c}} \mathbf{Q}^{a \alpha}{ }^{\left(5_{120}\right)} \mathcal{D}^{b}
$$

(j)

$$
\frac{15}{16} \epsilon_{i j k l m} \mathrm{M}^{i j} \mathrm{M}^{k l} \mathrm{H}^{(120) x} \mathbf{H}_{x}^{(210) m}=\frac{15}{4} \mathcal{S}_{24_{210}} \epsilon_{a b} \mathbf{U}_{\alpha}^{c} \mathbf{Q}^{a \alpha\left(5_{120}\right)} \mathcal{D}^{b}
$$

(k)

$$
\begin{aligned}
-15 \mathrm{M}^{i j} \mathrm{M}_{j} \mathrm{H}_{y z}^{(120) x} \mathrm{H}_{i x}^{(210) y z}=- & 15\left[\mathrm{M}^{a b} \mathrm{M}_{b} \mathrm{H}_{d e}^{(120) c} \mathrm{H}_{a c}^{(210) d e}-2 \mathrm{M}^{a b} \mathrm{M}_{b} \mathrm{H}_{\rho c}^{(120) \gamma} \mathrm{H}_{\gamma a}^{(210) \rho c}\right. \\
& \left.+\mathrm{M}^{a \alpha} \mathrm{M}_{\alpha} \mathrm{H}_{d e}^{(120) c} \mathrm{H}_{a c}^{(210) d e}-2 \mathrm{M}^{a \alpha} \mathrm{M}_{\alpha} \mathrm{H}_{\rho c}^{(120) \gamma} \mathrm{H}_{\gamma a}^{(210) \rho c}\right] \\
+ & \cdots
\end{aligned}
$$

i.

$$
\mathrm{M}^{a b} \mathrm{M}_{b} \mathrm{H}_{d e}^{(120) c} \mathbf{H}_{a c}^{(210) d e}=-\mathcal{S}_{75_{210}} \epsilon^{a b} \mathbf{E}^{\mathrm{c}} \mathbf{L}_{a}{ }^{\left(\overline{45}_{120}\right)} \mathcal{D}_{b}
$$

ii.

$$
-2 \mathrm{M}^{a b} \mathrm{M}_{b} \mathrm{H}_{\rho c}^{(120) \gamma} \mathbf{H}_{\gamma a}^{(210) \rho c}=-\frac{1}{3} \mathcal{S}_{75_{210}} \epsilon^{a b} \mathbf{E}^{\mathrm{c}} \mathbf{L}_{a}{ }^{\left(\overline{45}_{120}\right)} \mathcal{D}_{b}
$$


iii.

$$
\mathrm{M}^{a \alpha} \mathrm{M}_{\alpha} \mathrm{H}_{d e}^{(120) c} \mathrm{H}_{a c}^{(210) d e}=\mathcal{S}_{75_{210}} \mathbf{D}_{\alpha}^{c} \mathbf{Q}^{a \alpha}{\left(\overline{45}_{120}\right)}_{\mathcal{D}_{a}}
$$

iv.

$$
-2 \mathrm{M}^{a \alpha} \mathrm{M}_{\alpha} \mathrm{H}_{\rho c}^{(120) \gamma} \mathbf{H}_{\gamma a}^{(210) \rho c}=\frac{1}{3} \mathcal{S}_{75_{210}} \mathbf{D}_{\alpha}^{c} \mathbf{Q}^{a \alpha\left(\overline{45}_{120}\right)} \mathcal{D}_{a}
$$

(l)

$10 \mathrm{M}^{i j} \mathrm{M}_{j} \mathrm{H}_{i y}^{(120) x} \mathrm{H}_{x}^{(210) y}=10\left[\mathrm{M}^{a b} \mathrm{M}_{b} \mathrm{H}_{a b}^{(120) c} \mathrm{H}_{c}^{(210) b}-\mathrm{M}^{a b} \mathrm{M}_{b} \mathrm{H}_{\beta a}^{(120) \gamma} \mathrm{H}_{\gamma}^{(210) \beta}\right.$

$$
\left.+\mathrm{M}^{a \alpha} \mathrm{M}_{\alpha} \mathrm{H}_{a b}^{(120) c} \mathrm{H}_{c}^{(210) b}-\mathrm{M}^{a \alpha} \mathrm{M}_{\alpha} \mathrm{H}_{\beta a}^{(120) \gamma} \mathrm{H}_{\gamma}^{(210) \beta}\right]+\cdots
$$

i.

ii.

$$
\mathrm{M}^{a b} \mathrm{M}_{b} \mathrm{H}_{a b}^{(120) c} \mathrm{H}_{c}^{(210) b}=\frac{1}{2} \mathcal{S}_{24_{210}} \epsilon^{a b} \mathbf{E}^{\mathrm{c}} \mathbf{L}_{a}{ }^{\left(\overline{45}_{120}\right)} \mathcal{D}_{b}
$$

$$
-\mathbf{M}^{a b} \mathrm{M}_{b} \mathbf{H}_{\beta a}^{(120) \gamma} \mathbf{H}_{\gamma}^{(210) \beta}=\frac{1}{3} \mathcal{S}_{24_{210}} \epsilon^{a b} \mathbf{E}^{\mathrm{c}} \mathbf{L}_{a}{ }^{\left(\overline{45}_{120}\right)} \mathcal{D}_{b}
$$

iii.

$$
\mathbf{M}^{a \alpha} \mathbf{M}_{\alpha} \mathrm{H}_{a b}^{(120) c} \mathrm{H}_{c}^{(210) b}=-\frac{1}{2} \mathcal{S}_{24_{210}} \mathbf{D}_{\alpha}^{\mathrm{c}} \mathbf{Q}^{a \alpha}\left(\overline{(45}_{120}\right) \mathcal{D}_{a}
$$

iv.

$$
-\mathbf{M}^{a \alpha} \mathbf{M}_{\alpha} \mathbf{H}_{\beta a}^{(120) \gamma} \mathbf{H}_{\gamma}^{(210) \beta}=-\frac{1}{3} \mathcal{S}_{24_{210}} \mathbf{D}_{\alpha}^{\mathrm{c}} \mathbf{Q}^{a \alpha\left(\overline{45}_{120}\right)} \mathcal{D}_{a}
$$

(m)

$$
\begin{aligned}
-\frac{5}{2} \mathrm{M}^{i j} \mathrm{M}_{j} \mathrm{H}_{x}^{(120)} \mathrm{H}_{i}^{(210) x}=-\frac{5}{2}[ & \frac{1}{2} \mathcal{S}_{24_{210}} \epsilon^{a b} \mathbf{E}^{\mathrm{c}} \mathbf{L}_{a}{ }^{\left(\overline{5}_{120}\right)} \mathcal{D}_{b} \\
& \left.-\frac{1}{2} \mathcal{S}_{24_{210}} \mathbf{D}_{\alpha}^{\mathrm{c}} \mathbf{Q}^{a \alpha}{ }^{\left(\overline{(}_{120}\right)} \mathcal{D}_{a}+\cdots\right]
\end{aligned}
$$

(n)

$$
\begin{aligned}
-20 \mathrm{M}^{i j} \mathrm{M}_{k} \mathrm{H}_{j x}^{(120) k} \mathrm{H}_{i}^{(210) x}=-20[ & {\left[\mathrm{M}^{a c} \mathrm{M}_{d} \mathrm{H}_{c b}^{(120) d} \mathrm{H}_{a}^{(210) b}+\mathrm{M}^{a \alpha} \mathrm{M}_{\beta} \mathrm{H}_{\alpha b}^{(120 \beta} \mathrm{H}_{a}^{(210) b}\right.} \\
& \left.-\mathrm{M}^{a \alpha} \mathrm{M}_{\gamma} \mathrm{H}_{a \beta}^{(120) \gamma} \mathrm{H}_{\alpha}^{(210) \beta}\right]+\cdots
\end{aligned}
$$

i.

$$
\mathrm{M}^{a c} \mathrm{M}_{d} \mathrm{H}_{c b}^{(120) d} \mathrm{H}_{a}^{(210) b}=-\mathcal{S}_{24_{210}} \epsilon^{a b} \mathbf{E}^{c} \mathbf{L}_{a}^{\left(\overline{45}_{120}\right)} \mathcal{D}_{b}
$$

ii.

iii.

$$
\mathbf{M}^{a \alpha} \mathbf{M}_{\beta} \mathrm{H}_{\alpha b}^{(120 \beta} \mathbf{H}_{a}^{(210) b}=-\frac{1}{6} \mathcal{S}_{24_{210}} \mathbf{D}_{\alpha}^{c} \mathbf{Q}^{a \alpha}\left(\overline{45}_{120} \mathcal{D}_{a}\right.
$$

$$
-\mathrm{M}^{a \alpha} \mathrm{M}_{\gamma} \mathrm{H}_{a \beta}^{(120) \gamma} \mathrm{H}_{\alpha}^{(210) \beta}=\frac{1}{9} \mathcal{S}_{24_{210}} \mathbf{D}_{\alpha}^{\mathrm{c}} \mathbf{Q}^{a \alpha\left(\overline{45}_{120}\right)} \mathcal{D}_{a}
$$

(o)

$$
\begin{aligned}
-30 \mathrm{M}^{i j} \mathrm{M}_{k} \mathrm{H}_{i j}^{(120) x} \mathrm{H}_{x}^{(210) k}=-30[ & \mathcal{S}_{24_{210}} \epsilon^{a b} \mathbf{E}^{\mathrm{c}} \mathbf{L}_{a}{ }^{\left(\overline{45}_{120}\right)} \mathcal{D}_{b} \\
& \left.-\frac{2}{9} \mathcal{S}_{24_{210}} \mathbf{D}_{\alpha}^{\mathrm{c}} \mathbf{Q}^{a \alpha\left(\overline{45}_{120}\right)} \mathcal{D}_{a}+\cdots\right]
\end{aligned}
$$


(p)

$$
\begin{aligned}
30 \mathrm{M}^{i j} \mathrm{M}_{k} \mathrm{H}_{x y}^{(120) k} \mathrm{H}_{i j}^{(210) x y}=30[ & -2 \mathcal{S}_{75_{210}} \epsilon^{a b} \mathbf{E}^{c} \mathbf{L}_{a}{ }^{\left(\overline{45}_{120}\right)} \mathcal{D}_{b} \\
& \left.+\frac{2}{9} \mathcal{S}_{75_{210}} \mathbf{D}_{\alpha}^{\mathrm{c}} \mathbf{Q}^{a \alpha\left(\overline{45}_{120}\right)} \mathcal{D}_{a}+\cdots\right]
\end{aligned}
$$

(q)

$$
\begin{aligned}
3 \mathrm{M}^{i j} \mathrm{M}_{k} \mathrm{H}_{i j}^{(120) k} \mathrm{H}^{(210)}=3[ & -2 \mathcal{S}_{1_{210}} \epsilon^{a b} \mathbf{E}^{\mathrm{c}} \mathbf{L}_{a}{ }^{\left(\overline{45}_{120}\right)} \mathcal{D}_{b} \\
& \left.-\frac{2}{3} \mathcal{S}_{1_{210}} \mathbf{D}_{\alpha}^{\mathrm{c}} \mathbf{Q}^{a \alpha\left(\overline{45}_{120}\right)} \mathcal{D}_{a}+\cdots\right]
\end{aligned}
$$

(r)

$$
\begin{aligned}
-10 \mathrm{M}^{i j} \mathrm{M}_{k} \mathrm{H}_{i}^{(120)} \mathrm{H}_{j}^{(210) k}=-10 & {\left[\frac{1}{2} \mathcal{S}_{24_{210}} \epsilon^{a b} \mathbf{E}^{\mathrm{c}} \mathbf{L}_{a}{ }^{\left(\overline{5}_{120}\right)} \mathcal{D}_{b}\right.} \\
& \left.+\frac{1}{3} \mathcal{S}_{24_{210}} \mathbf{D}_{\alpha}^{\mathrm{c}} \mathbf{Q}^{a \alpha\left(\overline{5}_{120}\right)} \mathcal{D}_{a}+\cdots\right]
\end{aligned}
$$

(s)

$$
\begin{aligned}
-15 \mathrm{M}^{i j} \mathrm{M}_{k} \mathrm{H}_{x}^{(120)} \mathrm{H}_{i j}^{(210) k x}=-15 & {\left[\mathcal{S}_{75_{210}} \epsilon^{a b} \mathbf{E}^{\mathrm{c}} \mathbf{L}_{a}{ }^{\left(\overline{5}_{120}\right)} \mathcal{D}_{b}\right.} \\
& \left.+\frac{1}{3} \mathcal{S}_{75_{210}} \mathbf{D}_{\alpha}^{\mathrm{c}} \mathbf{Q}^{a \alpha\left(\overline{5}_{120}\right)} \mathcal{D}_{a}+\cdots\right]
\end{aligned}
$$

Thus,

$$
\begin{aligned}
& W_{4}^{(2)}=-i \frac{f^{(2)}}{5 ! M_{c}}\left[\left(\frac{20}{3} \mathcal{S}_{75_{210}}{ }^{\left(45_{120}\right)} \mathcal{D}^{b}+\frac{25}{9} \mathcal{S}_{24_{210}}{ }^{\left(45_{120}\right)} \mathcal{D}^{b}\right.\right. \\
& \left.+\frac{15}{4} \mathcal{S}_{24_{210}}{ }^{\left(5_{120}\right)} \mathcal{D}^{b}-\frac{3}{2} \mathcal{S}_{1_{210}}{ }^{\left(5_{120}\right)} \mathcal{D}^{b}\right) \epsilon_{a b} \mathbf{U}_{\alpha}^{c} \mathbf{Q}^{a \alpha} \\
& +\left(-\frac{40}{3} \mathcal{S}_{75_{210}}{ }^{(\overline{45}}{ }^{120}\right) \mathcal{D}_{a}-\frac{5}{9} \mathcal{S}_{24_{210}}{ }^{\left({ }^{45}{ }_{120}\right)} \mathcal{D}_{a}-\frac{25}{12} \mathcal{S}_{24_{210}}{ }^{\left(\overline{5}_{120}\right)} \mathcal{D}_{a} \\
& \left.-2 \mathcal{S}_{1_{210}}{ }^{\left(\overline{45}_{120}\right)} \mathcal{D}_{a}-5 \mathcal{S}_{75_{210}}{ }^{\left(\overline{5}_{120}\right)} \mathcal{D}_{a}\right) \mathbf{D}_{\alpha}^{\mathrm{c}} \mathbf{Q}^{a \alpha} \\
& +\left(-40 \mathcal{S}_{75_{210}}{ }^{\left(\overline{45}_{120}\right)} \mathcal{D}_{b}-\frac{5}{3} \mathcal{S}_{24_{210}}{ }^{\left(\overline{45}_{120}\right)} \mathcal{D}_{b}-\frac{25}{4} \mathcal{S}_{24_{210}}{ }^{\left(\overline{5}_{120}\right)} \mathcal{D}_{b}\right. \\
& \left.\left.\left.-6 \mathcal{S}_{1_{210}}{ }^{\left(\overline{45}_{120}\right)} \mathcal{D}_{b}-15 \mathcal{S}_{75_{210}}{ }^{\left({ }_{1}\right.}{ }^{20}\right) \mathcal{D}_{b}\right) \epsilon^{a b} \mathbf{E}^{c} \mathbf{L}_{a}+\cdots\right] \text {. }
\end{aligned}
$$


Usage of eqs. (2.5), (A.2) and (A.3) gives

$$
\begin{aligned}
{ }^{\left(5_{120}\right)} \mathcal{D}^{5} & =\frac{4}{\sqrt{3}}{ }^{\left(5_{120}\right)} \mathrm{D}^{5}=\frac{4}{\sqrt{3}} U_{d_{31}}\left\langle H_{u}\right\rangle+\cdots, \\
{ }^{\left(\overline{5}_{120}\right)} \mathcal{D}_{5} & =\frac{4}{\sqrt{3}}{ }^{\left(\overline{5}_{120}\right)} \mathrm{D}_{5}=\frac{4}{\sqrt{3}} V_{d_{31}}\left\langle H_{d}\right\rangle+\cdots, \\
{ }^{\left(45_{120}\right)} \mathcal{D}^{5} & =\frac{1}{\sqrt{2}}{ }^{\left(45_{120}\right)} \mathrm{D}^{5}=\frac{1}{\sqrt{2}} U_{d_{61}}\left\langle H_{u}\right\rangle+\cdots, \\
\left(\overline{45}_{120}\right) \mathcal{D}_{5} & =\frac{1}{\sqrt{2}}{ }^{\left(\overline{45}_{120}\right)} \mathrm{D}_{5}=\frac{1}{\sqrt{2}} V_{d_{61}}\left\langle H_{d}\right\rangle+\cdots,
\end{aligned}
$$

and finally making use of eqs. (A.5) and (A.6), gives eqs. (4.8)-(4.10).

3. Finally we compute the contributions to the third generation Yukawas from eq. (4.3).

$$
\begin{aligned}
W_{4}^{(3)}= & \frac{f^{(3)}}{M_{c}}\left\langle\Psi_{(+)}^{*}\left|B \Gamma_{\mu}\right| \Psi_{(+)}\right\rangle \Sigma_{\rho \sigma \lambda} \Phi_{\rho \sigma \lambda \mu} \\
=-\frac{3 i}{64} \frac{f^{(3)}}{M_{c}}\left[\epsilon_{i j k l m} \mathrm{M}^{i j} \mathrm{M}^{k l} \mathrm{H}_{c}^{(120) a b} \mathrm{H}_{a b}^{(210) c m}+2 \epsilon_{i j k l m} \mathrm{M}^{i j} \mathrm{M}^{k l} \mathrm{H}_{\beta}^{(120) a \alpha} \mathrm{H}_{a \alpha}^{(210) \beta m}\right. & \\
& +\frac{2}{3} \epsilon_{i j k l m} \mathrm{M}^{i j} \mathrm{M}^{k l} \mathrm{H}_{a}^{(120) m b} \mathrm{H}_{b}^{(210) a}+\frac{2}{3} \epsilon_{i j k l m} \mathrm{M}^{i j} \mathrm{M}^{k l} \mathrm{H}_{\alpha}^{(120) m \beta} \mathrm{H}_{\beta}^{(210) \alpha} \\
& \left.-\frac{1}{2} \epsilon_{i j k l m} \mathrm{M}^{i j} \mathrm{M}^{k l} \mathrm{H}^{(120) a} \mathrm{H}_{a}^{(210) m}-\frac{1}{10} \epsilon_{i j k l m} \mathrm{M}^{i j} \mathrm{M}^{k l} \mathrm{H}^{(120) m} \mathrm{H}^{(210)}+\cdots\right] \\
-\frac{3 i}{8} \frac{f^{(3)}}{M_{c}} & {\left[\mathrm{M}_{i} \mathrm{M}^{i j} \mathrm{H}_{a b}^{(120) c} \mathrm{H}_{c j}^{(210) a b}+2 \mathrm{M}_{i} \mathrm{M}^{i j} \mathrm{H}_{\alpha a}^{(120) \beta} \mathrm{H}_{\beta j}^{(210) \alpha a}+\frac{2}{3} \mathrm{M}_{i} \mathrm{M}^{i j} \mathrm{H}_{j a}^{(120) b} \mathrm{H}_{b}^{(210) a}\right.} \\
& +\frac{2}{3} \mathrm{M}_{i} \mathrm{M}^{i j} \mathrm{H}_{j \alpha}^{(120) \beta} \mathrm{H}_{\beta}^{(210) \alpha}-\frac{1}{2} \mathrm{M}_{i} \mathrm{M}^{i j} \mathrm{H}_{a}^{(120)} \mathrm{H}_{j}^{(210) a}-\frac{1}{10} \mathrm{M}_{i} \mathrm{M}^{i j} \mathrm{H}_{j}^{(120)} \mathrm{H}^{(210)} \\
+ & \cdots]
\end{aligned}
$$

$$
\begin{aligned}
\epsilon_{i j k l m} \mathrm{M}^{i j} \mathrm{M}^{k l} \mathrm{H}_{c}^{(120) a b} \mathrm{H}_{a b}^{(210) c m} & =8 \mathcal{S}_{75_{210}} \mathbf{U}_{\alpha}^{\mathrm{c}} \mathbf{U}^{\alpha}\left(45_{120}\right) \mathcal{D}^{5} \\
2 \epsilon_{i j k l m} \mathrm{M}^{i j} \mathrm{M}^{k l} \mathrm{H}_{\beta}^{(120) a \alpha} \mathrm{H}_{a \alpha}^{(210) \beta m} & =\frac{8}{3} \mathcal{S}_{75_{210}} \mathbf{U}_{\alpha}^{\mathrm{c}} \mathbf{U}^{\alpha}\left(45_{120}\right) \mathcal{D}^{5} \\
\frac{2}{3} \epsilon_{i j k l m} \mathrm{M}^{i j} \mathrm{M}^{k l} \mathrm{H}_{a}^{(120) m b} \mathrm{H}_{b}^{(210) a} & =\frac{8}{3} \mathcal{S}_{24_{210}} \mathbf{U}_{\alpha}^{\mathrm{c}} \mathbf{U}^{\alpha}\left({ }^{\left(45_{120}\right)} \mathcal{D}^{5}\right. \\
\frac{2}{3} \epsilon_{i j k l m} \mathrm{M}^{i j} \mathrm{M}^{k l} \mathrm{H}_{\alpha}^{(120) m \beta} \mathrm{H}_{\beta}^{(210) \alpha} & =\frac{16}{9} \mathcal{S}_{24_{210}} \mathbf{U}_{\alpha}^{\mathrm{c}} \mathbf{U}^{\alpha}\left(45_{120}\right) \mathcal{D}^{5} \\
-\frac{1}{2} \epsilon_{i j k l m} \mathrm{M}^{i j} \mathrm{M}^{k l} \mathrm{H}^{(120) a} \mathrm{H}_{a}^{(210) m} & =-2 \mathcal{S}_{24_{210}} \mathbf{U}_{\alpha}^{\mathrm{c}} \mathbf{U}^{\alpha\left(5_{120}\right)} \mathcal{D}^{5} \\
-\frac{1}{10} \epsilon_{i j k l m} \mathrm{M}^{i j} \mathrm{M}^{k l} \mathrm{H}^{(120) m} \mathrm{H}^{(210)} & =\frac{4}{5} \mathcal{S}_{1_{210}} \mathbf{U}_{\alpha}^{\mathrm{c}} \mathbf{U}^{\alpha\left(5_{120}\right) \mathcal{D}^{5}}
\end{aligned}
$$




$$
\begin{gathered}
\mathrm{M}_{i} \mathrm{M}^{i j} \mathrm{H}_{a b}^{(120) c} \mathrm{H}_{c j}^{(210) a b}=-\mathcal{S}_{75_{210}} \mathbf{E}^{\mathrm{c}} \mathbf{E}^{\left(\overline{45}_{120}\right)} \mathcal{D}_{5}+\mathcal{S}_{75_{210}} \mathbf{D}_{\alpha}^{\mathrm{c}} \mathbf{D}^{\alpha\left(\overline{45}_{120}\right)} \mathcal{D}_{5} \\
2 \mathrm{M}_{i} \mathrm{M}^{i j} \mathrm{H}_{\alpha a}^{(120) \beta} \mathrm{H}_{\beta j}^{(210) \alpha a}=-\frac{1}{3} \mathcal{S}_{75_{210}} \mathbf{E}^{\mathrm{c}} \mathbf{E}^{\left(\overline{45}_{120}\right)} \mathcal{D}_{5}+\frac{1}{3} \mathcal{S}_{75_{210}} \mathbf{D}_{\alpha}^{\mathrm{c}} \mathbf{D}^{\alpha\left(\overline{45}_{120}\right)} \mathcal{D}_{5} \\
\frac{2}{3} \mathrm{M}_{i} \mathrm{M}^{i j} \mathrm{H}_{j a}^{(120) b} \mathrm{H}_{b}^{(210) a}=-\frac{1}{3} \mathcal{S}_{24_{210}} \mathbf{E}^{\mathrm{c}} \mathbf{E}^{\left(\overline{45}_{120}\right)} \mathcal{D}_{5}+\frac{1}{3} \mathcal{S}_{24_{210}} \mathbf{D}_{\alpha}^{\mathrm{c}} \mathbf{D}^{\alpha\left(\overline{45}_{120}\right)_{\mathcal{D}_{5}}} \\
\frac{2}{3} \mathrm{M}_{i} \mathrm{M}^{i j} \mathrm{H}_{j \alpha}^{(120) \beta} \mathrm{H}_{\beta}^{(210) \alpha}=-\frac{2}{9} \mathcal{S}_{24_{210}} \mathbf{E}^{\mathrm{c}} \mathbf{E}^{\left(\overline{45}_{120}\right)} \mathcal{D}_{5}+\frac{2}{9} \mathcal{S}_{24_{210}} \mathbf{D}_{\alpha}^{\mathrm{c}} \mathbf{D}^{\alpha\left(\overline{45}_{120}\right)} \mathcal{D}_{5} \\
-\frac{1}{2} \mathrm{M}_{i} \mathrm{M}^{i j} \mathrm{H}_{a}^{(120)} \mathrm{H}_{j}^{(210) a}=\frac{1}{4} \mathcal{S}_{24_{210}} \mathbf{E}^{\mathrm{c}} \mathbf{E}^{\left(\overline{5}_{120}\right)} \mathcal{D}_{5}-\frac{1}{4} \mathcal{S}_{24_{210}} \mathbf{D}_{\alpha}^{\mathrm{c}} \mathbf{D}^{\alpha\left(\overline{5}_{120}\right)} \mathcal{D}_{5} \\
-\frac{1}{10} \mathrm{M}_{i} \mathrm{M}^{i j} \mathrm{H}_{j}^{(120)} \mathrm{H}^{(210)}=-\frac{1}{10} \mathcal{S}_{1_{210}} \mathbf{E}^{\mathrm{c}} \mathbf{E}^{\left(\overline{5}_{120}\right)} \mathcal{D}_{5}+\frac{1}{10} \mathcal{S}_{1_{210}} \mathbf{D}_{\alpha}^{\mathrm{c}} \mathbf{D}^{\alpha\left(\overline{5}_{120}\right)} \mathcal{D}_{5}
\end{gathered}
$$

Open Access. This article is distributed under the terms of the Creative Commons Attribution License (CC-BY 4.0), which permits any use, distribution and reproduction in any medium, provided the original author(s) and source are credited.

\section{References}

[1] H. Georgi, Constructed the SO(10) grand unified theory, in Particles and fields - 1974, proc. of the meeting of the APS division of particles and fields, Williamsburg, VA, U.S.A., C.E. Carlson ed., AIP, New York, U.S.A. (1975), pg. 575.

[2] H. Fritzsch and P. Minkowski, Unified interactions of leptons and hadrons, Annals Phys. 93 (1975) 193 [INSPIRE].

[3] T.E. Clark, T.-K. Kuo and N. Nakagawa, A SO(10) supersymmetric grand unified theory, Phys. Lett. B 115 (1982) 26 [inSPIRE].

[4] C.S. Aulakh and R.N. Mohapatra, Implications of supersymmetric $\mathrm{SO}(10)$ grand unification, Phys. Rev. D 28 (1983) 217 [InSPIRE].

[5] K.S. Babu and R.N. Mohapatra, Predictive neutrino spectrum in minimal SO(10) grand unification, Phys. Rev. Lett. 70 (1993) 2845 [hep-ph/9209215] [INSPIRE].

[6] P. Nath and R.M. Syed, Analysis of couplings with large tensor representations in $\mathrm{SO}(2 N)$ and proton decay, Phys. Lett. B 506 (2001) 68 [Erratum ibid. 508 (2001) 216] [hep-ph/0103165] [InSPIRE].

[7] P. Nath and R.M. Syed, Complete cubic and quartic couplings of 16 and $\overline{16}$ in $\mathrm{SO}(10)$ unification, Nucl. Phys. B 618 (2001) 138 [hep-th/0109116] [INSPIRE].

[8] P. Nath and R.M. Syed, Coupling the supersymmetric 210 vector multiplet to matter in SO(10), Nucl. Phys. B 676 (2004) 64 [hep-th/0310178] [INSPIRE]. 
[9] C.S. Aulakh, B. Bajc, A. Melfo, G. Senjanović and F. Vissani, The minimal supersymmetric grand unified theory, Phys. Lett. B 588 (2004) 196 [hep-ph/0306242] [InSPIRE].

[10] B. Bajc, A. Melfo, G. Senjanović and F. Vissani, The minimal supersymmetric grand unified theory. 1. Symmetry breaking and the particle spectrum, Phys. Rev. D 70 (2004) 035007 [hep-ph/0402122] [INSPIRE].

[11] C.S. Aulakh and A. Girdhar, $\mathrm{SO}(10)$ MSGUT: spectra, couplings and threshold effects, Nucl. Phys. B 711 (2005) 275 [hep-ph/0405074] [InSPIRE].

[12] C.S. Aulakh and S.K. Garg, The new minimal supersymmetric GUT: spectra, RG analysis and fermion fits, Nucl. Phys. B 857 (2012) 101 [arXiv:0807.0917] [INSPIRE].

[13] P. Nath and P. Fileviez Perez, Proton stability in grand unified theories, in strings and in branes, Phys. Rept. 441 (2007) 191 [hep-ph/0601023] [INSPIRE].

[14] V. Braun, Y.-H. He, B.A. Ovrut and T. Pantev, The exact MSSM spectrum from string theory, JHEP 05 (2006) 043 [hep-th/0512177] [INSPIRE].

[15] V. Bouchard, M. Cvetič and R. Donagi, Tri-linear couplings in an heterotic minimal supersymmetric standard model, Nucl. Phys. B $\mathbf{7 4 5}$ (2006) 62 [hep-th/0602096] [INSPIRE].

[16] L.B. Anderson, J. Gray, Y.-H. He and A. Lukas, Exploring positive monad bundles and a new heterotic Standard Model, JHEP 02 (2010) 054 [arXiv:0911.1569] [INSPIRE].

[17] V. Bouchard and R. Donagi, An SU(5) heterotic Standard Model, Phys. Lett. B 633 (2006) 783 [hep-th/0512149] [INSPIRE].

[18] S.K. Dimopoulos and F. Wilczek, Incomplete multiplets in supersymmetric unified models, Tech. Rep. NSF-ITP-82-07, Calif. Univ. Santa Barbara Inst. Theor. Phys., Santa Barbara, CA, U.S.A. (1981).

[19] K.S. Babu and S.M. Barr, Natural gauge hierarchy in SO(10), Phys. Rev. D 50 (1994) 3529 [hep-ph/9402291] [INSPIRE].

[20] D.-G. Lee and R.N. Mohapatra, Natural doublet-triplet splitting in supersymmetric $\mathrm{SO}(10)$ models, Phys. Lett. B 324 (1994) 376 [hep-ph/9310371] [INSPIRE].

[21] Y.-K. Chen and D.-X. Zhang, A renormalizable supersymmetric $\mathrm{SO}(10)$ model with natural doublet-triplet splitting, JHEP 01 (2015) 025 [arXiv:1410.5625] [INSPIRE].

[22] A. Masiero, D.V. Nanopoulos, K. Tamvakis and T. Yanagida, Naturally massless Higgs doublets in supersymmetric SU(5), Phys. Lett. B 115 (1982) 380 [InSPIRE].

[23] B. Grinstein, A supersymmetric SU(5) gauge theory with no gauge hierarchy problem, Nucl. Phys. B 206 (1982) 387 [INSPIRE].

[24] K.S. Babu, I. Gogoladze and Z. Tavartkiladze, Missing partner mechanism in $\mathrm{SO}(10)$ grand unification, Phys. Lett. B 655 (2007) 49 [hep-ph/0612315] [INSPIRE].

[25] K.S. Babu, I. Gogoladze, P. Nath and R.M. Syed, Variety of $\mathrm{SO}(10)$ GUTs with natural doublet-triplet splitting via the missing partner mechanism, Phys. Rev. D 85 (2012) 075002 [arXiv: 1112.5387] [INSPIRE].

[26] L. Du, X. Li and D.-X. Zhang, Proton decay in a supersymmetric $\mathrm{SO}(10)$ model with missing partner mechanism, JHEP 04 (2014) 027 [arXiv: 1312.1786] [INSPIRE].

[27] B. Ananthanarayan, G. Lazarides and Q. Shafi, Radiative electroweak breaking and sparticle spectroscopy with $\tan \beta \simeq m_{t} / m_{b}$, Phys. Lett. B 300 (1993) 245 [INSPIRE]. 
[28] P. Nath and R.M. Syed, An analysis of $B-L=-2$ operators from matter-Higgs interactions in a class of supersymmetric $\mathrm{SO}(10)$ models, Phys. Rev. D 93 (2016) 055005 [arXiv: 1508.00585] [INSPIRE].

[29] P. Nath, Hierarchies and textures in supergravity unification, Phys. Rev. Lett. 76 (1996) 2218 [hep-ph/9512415] [INSPIRE].

[30] R.N. Mohapatra and B. Sakita, $\mathrm{SO}(2 N)$ grand unification in an $\mathrm{SU}(N)$ basis, Phys. Rev. $D$ 21 (1980) 1062 [INSPIRE].

[31] P. Nath and R.M. Syed, Couplings of vector-spinor representation for $\mathrm{SO}(10)$ model building, JHEP 02 (2006) 022 [hep-ph/0511172] [INSPIRE].

[32] ATLAS collaboration, Observation of a new particle in the search for the Standard Model Higgs boson with the ATLAS detector at the LHC, Phys. Lett. B 716 (2012) 1 [arXiv: 1207.7214] [INSPIRE].

[33] CMS collaboration, Observation of a new boson at a mass of $125 \mathrm{GeV}$ with the CMS experiment at the LHC, Phys. Lett. B 716 (2012) 30 [arXiv:1207.7235] [InSPIRE].

[34] Particle Data Group collaboration, Review of particle physics, Phys. Rev. D 98 (2018) 030001 [INSPIRE].

[35] A.H. Chamseddine, R.L. Arnowitt and P. Nath, Locally supersymmetric grand unification, Phys. Rev. Lett. 49 (1982) 970 [INSPIRE].

[36] P. Nath, R.L. Arnowitt and A.H. Chamseddine, Gauge hierarchy in supergravity GUTs, Nucl. Phys. B 227 (1983) 121 [inSPIRE].

[37] L.J. Hall, J.D. Lykken and S. Weinberg, Supergravity as the messenger of supersymmetry breaking, Phys. Rev. D 27 (1983) 2359 [INSPIRE].

[38] P. Nath, Supersymmetry, supergravity and unification, Cambridge University Press, Cambridge, U.K. (2016) [INSPIRE].

[39] W. Porod, SPheno, a program for calculating supersymmetric spectra, SUSY particle decays and SUSY particle production at $e^{+} e^{-}$colliders, Comput. Phys. Commun. 153 (2003) 275 [hep-ph/0301101] [INSPIRE].

[40] W. Porod and F. Staub, SPheno 3.1: extensions including flavour, CP-phases and models beyond the MSSM, Comput. Phys. Commun. 183 (2012) 2458 [arXiv:1104.1573] [INSPIRE].

[41] F. Staub and W. Porod, Improved predictions for intermediate and heavy supersymmetry in the MSSM and beyond, Eur. Phys. J. C 77 (2017) 338 [arXiv:1703.03267] [INSPIRE].

[42] K.S. Babu, I. Gogoladze, P. Nath and R.M. Syed, A unified framework for symmetry breaking in $\mathrm{SO}(10)$, Phys. Rev. D 72 (2005) 095011 [hep-ph/0506312] [INSPIRE].

[43] K.S. Babu, I. Gogoladze, P. Nath and R.M. Syed, Fermion mass generation in $\mathrm{SO}(10)$ with a unified Higgs sector, Phys. Rev. D 74 (2006) 075004 [hep-ph/0607244] [INSPIRE].

[44] P. Nath and R.M. Syed, Suppression of Higgsino mediated proton decay by cancellations in GUTs and strings, Phys. Rev. D 77 (2008) 015015 [arXiv:0707.1332] [INSPIRE].

[45] P. Nath and R.M. Syed, Yukawa couplings and quark and lepton masses in an $\mathrm{SO}(10)$ model with a unified Higgs sector, Phys. Rev. D 81 (2010) 037701 [arXiv:0909.2380] [INSPIRE].

[46] M.A. Ajaib, I. Gogoladze and Q. Shafi, Sparticle spectroscopy from $\mathrm{SO}(10)$ GUT with a unified Higgs sector, Phys. Rev. D 88 (2013) 095019 [arXiv:1307.4882] [INSPIRE]. 
[47] T. Dasgupta, P. Mamales and P. Nath, Effects of gravitational smearing on predictions of supergravity grand unification, Phys. Rev. D 52 (1995) 5366 [hep-ph/9501325] [INSPIRE].

[48] A. Aboubrahim and P. Nath, Supersymmetry at a $28 \mathrm{TeV}$ hadron collider: HE-LHC, Phys. Rev. D 98 (2018) 015009 [arXiv: 1804.08642] [INSPIRE].

[49] G. Bélanger, F. Boudjema, A. Pukhov and A. Semenov, MicrOMEGAs4.1: two dark matter candidates, Comput. Phys. Commun. 192 (2015) 322 [arXiv:1407.6129] [INSPIRE].

[50] Planck collaboration, Planck 2018 results. VI. Cosmological parameters, Astron. Astrophys. 641 (2020) A6 [arXiv: 1807.06209] [INSPIRE].

[51] D. Feldman, Z. Liu, P. Nath and G. Peim, Multicomponent dark matter in supersymmetric hidden sector extensions, Phys. Rev. D 81 (2010) 095017 [arXiv: 1004.0649] [INSPIRE].

[52] D. Feldman, P. Fileviez Perez and P. Nath, R-parity conservation via the Stückelberg mechanism: LHC and dark matter signals, JHEP 01 (2012) 038 [arXiv:1109.2901] [INSPIRE].

[53] A. Aboubrahim and P. Nath, LHC phenomenology with hidden sector dark matter: a long-lived stau and heavy Higgs in an observable range, in Meeting of the division of particles and fields of the American Physical Society, (2019) [arXiv: 1909.08684] [INSPIRE].

[54] H. Baer, V. Barger, D. Sengupta and X. Tata, Is natural higgsino-only dark matter excluded?, Eur. Phys. J. C 78 (2018) 838 [arXiv:1803.11210] [INSPIRE].

[55] J. Halverson, C. Long and P. Nath, Ultralight axion in supersymmetry and strings and cosmology at small scales, Phys. Rev. D 96 (2017) 056025 [arXiv:1703.07779] [INSPIRE].

[56] A. Aboubrahim, W.-Z. Feng and P. Nath, Expanding the parameter space of natural supersymmetry, JHEP 04 (2020) 144 [arXiv: 2003.02267] [INSPIRE].

[57] B. Kaufman, P. Nath, B.D. Nelson and A.B. Spisak, Light stops and observation of supersymmetry at LHC run-II, Phys. Rev. D 92 (2015) 095021 [arXiv:1509.02530] [INSPIRE].

[58] A. Aboubrahim, P. Nath and A.B. Spisak, Stau coannihilation, compressed spectrum, and SUSY discovery potential at the LHC, Phys. Rev. D 95 (2017) 115030 [arXiv:1704.04669] [INSPIRE].

[59] A. Aboubrahim and P. Nath, Naturalness, the hyperbolic branch, and prospects for the observation of charged Higgs bosons at high luminosity LHC and 27 TeV LHC, Phys. Rev. D 98 (2018) 095024 [arXiv: 1810.12868] [INSPIRE].

[60] A. Aboubrahim and P. Nath, Mixed hidden sector-visible sector dark matter and observation of a CP odd Higgs boson at HL-LHC and HE-LHC, Phys. Rev. D 100 (2019) 015042 [arXiv: 1905.04601] [INSPIRE].

[61] CMS collaboration, Search for new physics in events with two low momentum opposite-sign leptons and missing transverse energy at $\sqrt{s}=13 \mathrm{TeV}$, Tech. Rep. CMS-PAS-SUS-16-048, CERN, Geneva, Switzerland (2017).

[62] CMS collaboration, Search for new physics in events with two soft oppositely charged leptons and missing transverse momentum in proton-proton collisions at $\sqrt{s}=13 \mathrm{TeV}$, Phys. Lett. B 782 (2018) 440 [arXiv:1801.01846] [INSPIRE]. 
[63] CMS collaboration, Search for supersymmetry with a compressed mass spectrum in the vector boson fusion topology with 1-lepton and 0-lepton final states in proton-proton collisions at $\sqrt{s}=13 \mathrm{TeV}$, JHEP 08 (2019) 150 [arXiv: 1905.13059] [INSPIRE].

[64] ATLAS collaboration, Search for chargino-neutralino production with mass splittings near the electroweak scale in three-lepton final states in $\sqrt{s}=13 \mathrm{TeV}$ pp collisions with the ATLAS detector, Phys. Rev. D 101 (2020) 072001 [arXiv:1912.08479] [INSPIRE].

[65] ATLAS collaboration, Search for the electroweak production of supersymmetric particles in $\sqrt{s}=8 \mathrm{TeV}$ pp collisions with the ATLAS detector, Phys. Rev. D 93 (2016) 052002 [arXiv: 1509.07152] [INSPIRE].

[66] ATLAS collaboration, Search for electroweak production of supersymmetric particles in final states with two or three leptons at $\sqrt{s}=13 \mathrm{TeV}$ with the ATLAS detector, Eur. Phys. J. C 78 (2018) 995 [arXiv: 1803.02762] [INSPIRE].

[67] J. Debove, B. Fuks and M. Klasen, Joint resummation for gaugino pair production at hadron colliders, Nucl. Phys. B 849 (2011) 64 [arXiv:1102.4422] [InSPIRE].

[68] B. Fuks, M. Klasen, D.R. Lamprea and M. Rothering, Precision predictions for electroweak superpartner production at hadron colliders with Resummino, Eur. Phys. J. C 73 (2013) 2480 [arXiv: 1304.0790] [INSPIRE].

[69] A. Buckley et al., LHAPDF6: parton density access in the LHC precision era, Eur. Phys. J. $C 75$ (2015) 132 [arXiv:1412.7420] [INSPIRE].

[70] T. Sjöstrand et al., An introduction to PYTHIA 8.2, Comput. Phys. Commun. 191 (2015) 159 [arXiv: 1410.3012] [INSPIRE].

[71] M. Cacciari, G.P. Salam and G. Soyez, FastJet user manual, Eur. Phys. J. C 72 (2012) 1896 [arXiv:1111.6097] [INSPIRE].

[72] M. Cacciari, G.P. Salam and G. Soyez, The anti- $k_{t}$ jet clustering algorithm, JHEP 04 (2008) 063 [arXiv:0802.1189] [INSPIRE].

[73] DELPHES 3 collaboration, DELPHES 3, a modular framework for fast simulation of a generic collider experiment, JHEP 02 (2014) 057 [arXiv:1307.6346] [INSPIRE].

[74] I. Antcheva et al., ROOT: a $C++$ framework for petabyte data storage, statistical analysis and visualization, Comput. Phys. Commun. 182 (2011) 1384 [INSPIRE].

[75] X. Cid Vidal et al., Report from working group 3: beyond the Standard Model physics at the HL-LHC and HE-LHC, CERN Yellow Rep. Monogr. 7 (2019) 585 [arXiv:1812.07831] [INSPIRE].

[76] M. Cepeda et al., Report from working group 2: Higgs physics at the HL-LHC and HE-LHC, CERN Yellow Rep. Monogr. 7 (2019) 221 [arXiv:1902.00134] [INSPIRE].

[77] Z.-Y. Chen, D.-X. Zhang and X.-Z. Bai, Couplings in renormalizable supersymmetric $\mathrm{SO}(10)$ models, Int. J. Mod. Phys. A 32 (2017) 1750207 [arXiv:1707.00580] [InSPIRE]. 